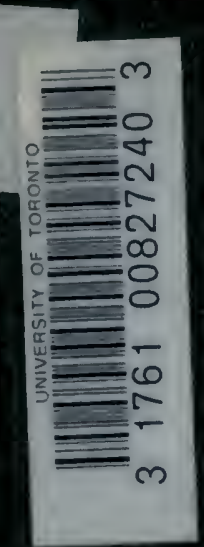




Digitized by the Internet Archive in 2008 with funding from Microsoft Corporation 
J

Q. B. E. Fennow

Cmplements of as admirer tone who recever hisfirst lessons in foresto undey your able grieteance.

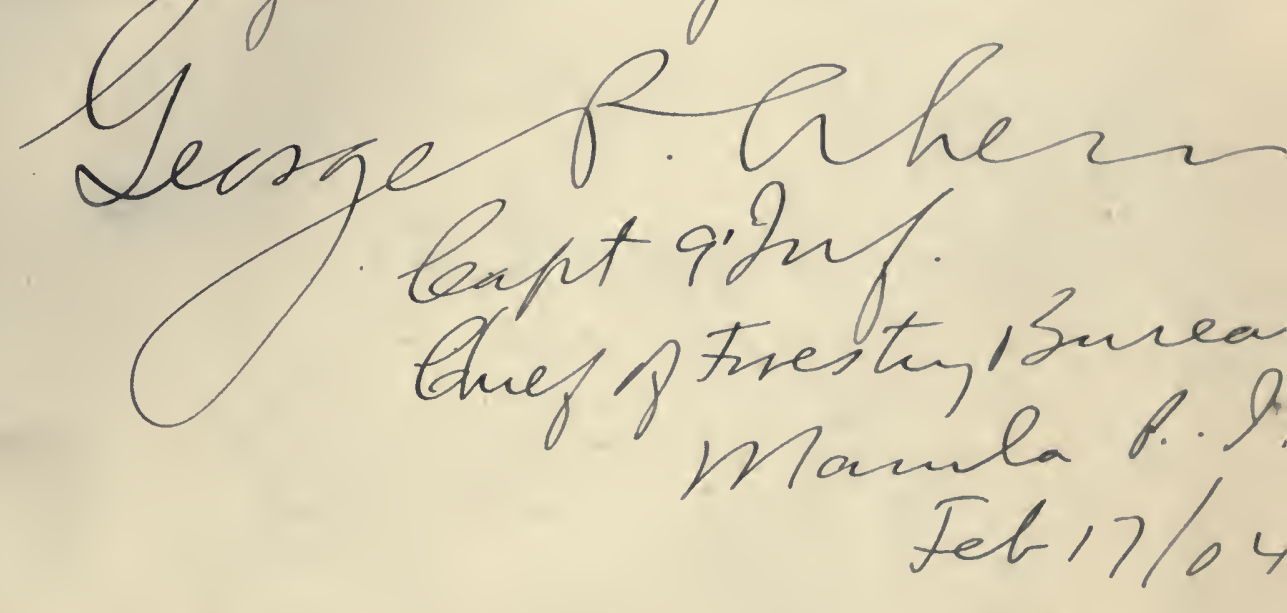

LIERARY

UNIVERSITY OF TOROWTO 





\section{DEPARTMENT OF THE INTERIOR,}

FORESTRY BUREAU—BULLETIN NO. I.

CAPTAIN George P. AHERn, Chief of Bureau.

\section{REPORT ON INVESTIGATIONS MADE IN JAVA IN THE YEAR 1902.}

I. REPOHT OF TIIP. II. PLANTA AHEIRIANA. II. THE METHOH OF WORK ON THE FOIRST HLOIRA OF JAVA. IV. THE HOTANICAL INSTITUTE AT IBUTENZOIR:

FIMIR D. MERRILL,

Botanist in the BUREAUS OF AGRICUlture AND Forestry.

ISSUED OCTOBER 15, 1903.

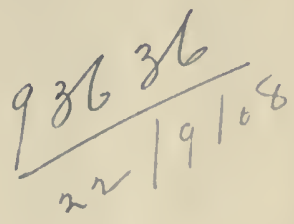

MANILA:

BUREAU OF PUBLIC PRINTING. 


$$
\begin{aligned}
& Q R \\
& 367 \\
& M 47 \\
& 1903
\end{aligned}
$$




\section{LETTER OF TRANSMITTAL.}

Dhpartaext of the Interior, Forestry Bureav, Manila, March 30, 1908.

SIm: I have the honor to transmit herewith the manuseript of a paper entitled "Report of Investigations made in Java in the Year 1902," by Elmer D. Merrill, Botanist, and recommend its publication as Bulletin No. 1.

Very respectfully,

George P. Ainers, Captain, Ninth U.S. Infantry,

Chief of Forestry Bureau.

Hon. Dean C. Worcester, Sccretary of the Interior. 


\section{LETTER OF SUBMITTAL.}

Departmext of the Ixterior, Forestry Bureau, Manila, December 3, 1902.

SIR: I have the honor to present herewith my report on the work accomplished in Java during the months of September, October, and November, 1902, under your letter of authorization and instruction, dated Manila, P. I., August 27, 1902.

Very respectfully,

Elmer D. Merimll,

Botanist, Bureaus of Agriculture and Forestry.

Captain Georese P. Ahern,

Chief of Forestry Bureau, Manila P. I. 


\section{ILLUSTRATIONS.}

Plate I. Fig. 1. The botanical inuseum.

Fig. 2. The botanical museum, rear view.

II. Fig. 1. The botanical museum, interior, main floor.

Fig. 2. The botanical museum, interior, from the balcony.

III. Fig. 1. Herbarium building, forest flora investigations.

Fig. 2. Laboratory for systematic botany, forest flora investigations.

IV. Fig. 1. Office of the experiment garden.

Fig. 2. Aroid house.

V. Fig. 1. The Cunarizm avenue.

Fig. 2. A palm avenue.

VI. Fig. 1. The new Canarium avenue.

Fig. 2. A view in the Pandumus section.

VII. Fig. 1. A view in the palm section.

Fig. 2. Method of labeling sections, and thickness of planting.

VIII. Figs. 1 and 2. The river in the garden.

IX. Fig. 1. The main driveway in the experiment garden.

Fig. 2. Young plants of Ficus elastica in the experiment garden.

x. Fig. 1. Gutta-percha trees in the experiment garden.

Fig. 2. Showing thickness of planting in the experiment garden. 



\title{
REPORT ON INVESTIGATIONS MADE IN JAVA IN THE YEAR I902.
}

\author{
By WLMER D. M ERRILL.
}

\section{REPORT OF TRIP.}

In accordance with my instructions, I left Manila on September 2, 1902, reaching Singapore on the morning of September 8. I left Singapore September 9, reaching Batavia on the morning of September 11, and as soon as practicable went to Buitenzorg, but being unable to secure hotel accommodations there, returned to Batavia. I remained in Batavia until the morning of September 15, on which date I returned to Buitenzorg. On my first visit to Buitenzorg, September 13, I called on Dr. Van Romburgh, Acting Director of the 's Lands Plantentuin, presented lim with Captain Ahern's letter, and outlined the work which I wished to accomplish. Dr. Van Romburgh very kindly placed at my disposal all the facilities of the herbarium, garden, and library, so that on my return on September 15 I was able to begin work immediately.

As the chief object of my trip was to identify the botanical specimens in the herbarium of the Forestry Bureau, attention was given first to this work, the results of which are given in Part II, Plantæ Ahernianæ.

In addition to determining the abore Forestry Bureau material, about 300 specimens of my own collection in the herbarium of the Bureau of Agriculture were identified.

'The time from September 15 to November 15, with the exception of two days spent in a trip to Batavia to interview Dr. Bruisma, inspectorgeneral of the forestry bureau for the Island of Java, was spent at Buitzenborg. This time was taken up for the most part in identifying the botanical material mentioned above, it being too limited to allow for the working out of all the eritical species. On November 14, having completed all the work possible in the time allotted to me, I again went to Batavia, sailing that night for Singapore, where 1 arrived on the morning of November 1\%. I was obliged to wait for a Manila steamer until November 24. On that date I took the S. S. Korat for Manila, via Labuan, reaching Manila December 2.

In addition to my major work in identifying the botanieal material mentioned above, a special report has been prepared (Part III) on Dr. Koorders's method of investigating the forest flora of Java, which includes 
the method of determining the rate of tree growth, prevalence of species of economic importance, etc. In Part IV there has been included notes on the botanical garden, library, laboratories, herbarium, etc. Extensive notes not included in this report were also compiled regarding publications, with special reference to those that apply to the Philippines, and much library work, looking up references to the Plhilippine flora, publications of new species from the Archipelago, etc., was done.

Some 600 specimens of the botanical material which were taken to Buitenzorg were in duplicate. These were presented to the herbarium of the botanical garden, and in exchange for this material a similar collection of authentically named species in the garden are being prepared for the Forestry Bureau of the Philippine Islands. About 200 specimens were prepared while at Buitenzorg.

The catalogue of the garden was carefully perused and approximately 1,000 species were specified, of which specimens were desired. These are now being prepared and will be sent later. All the specimens which were marked are trees or shrubs. The greater part of them are also found in the Philippines. This material will be of the greatest value as a guide in identifying future Philippine collections. In addition to specimens of the trees and shrubs, I also personally collected specimens from all the species of grasses growing in the gardens and was allowed to take these specimens from the duplicates of Dr. Koorders's Java collections. These grass specimens will be of very great interest to the herbarium of the Bureau of Agriculture.

From Mr. Bruisma a report of the work of the forestry burean, a catalogue of the library of the bureau, a copy of the forestry regulations, and maps showing the method of charting the "djati" (teak) forests were secured. This latter will be of special interest to the Bureau of Forestry.

At Buitenzorg a copy of the eatalogue of the library was olstained, and also as complete a set as possible of the publications of the 's Lands Plantentuin. These publications include the "Mededeelingen" (contributions; "Verslag" (annual reports): "Icones Bogoriensis," and the "Bulletin." Many of these publications are of the greatest importance for our work on the Philippine forest flora. Especially to be noted are the seven volumes of the "Mededeelingen" by Koorders and Valeton on the forest flora of Java. 'These publications were secured in exchange for the publications of the Forestry Bureau of the Philippine Islands, which Bureau has been placed on the permanent mailing list of the 's Iands Plantentuin.

A full set of photographs were made at Buitenzorg, showing the buildings and laboratories eonnected with the 's Lands Plantentuin, the interior of the botanical museum, iaboratories, etc., besides a series showing the general features of the botanical and experimental gardens.

During my stay at Singapore advantage was taken of the opportunity and some investigations were made of the botanieal garden there. Dr. 
H. N. Ridley, the director, very kindly placed the facilities of the institution at my disposal. As a result of this visit a large package of authentically named botanical material was received and arrangements were made for future exchange of botanical specimens.

\section{PLANTÆ AHERNIANÆ.}

INTIODUCTION.

The material upon which the present paper is based has been secured by the various field parties of the Forestry Bureau during the year 1901. and up to June, 1902. Realizing that little accurate work could be done on the many forestry problems presented in the Archipclago without first accurately identifying the component species of the forest flora, Capt. George P. Ahern, Chief of the Bureau, made an attempt to secure all the botanical material possible through his field parties, and, considering the conditions under which the work was done, met with considerable success. While it is unfortunate that the material could not have been collected under the direction of a trained botanist and properly cared for by such a person, still there is much of value in the collection, both from a scientific and an economic standpoint, as it forms the basis of our Philippine Herbarium, and enables us to accurately identify much of the material in the timber-testing laboratory of the Forestry Bureau.

The material considered in this paper, collected under the direction of Captain Ahern, has been credited to him, and the title of the present paper is derived from the name of the man who, although not a botanist, recognizes the pressing need and great value of thorough botanical work on the forest flora of this Archipelago.

Upon my appointment in the Bureau of Forestry in August, 1902, work was at once commenced arranging these specimens, completing the labels, etc. Difficulty was experienced, due to the fact that a large part of the material was without data other than the place of collection, and sometimes even this was wanting. Many of the packages had not been worked over, and as none of the specimens had been poisoned, much of the material was entirely destroyed by insects, while in other cases specimens that had not been properly dried had been rendered worthless by moisture. Much of the material was collected by persons who had little or no botanical training, and hence did not understand the necessity of securing complete botanical specimens. Accordingly, very few of the numbers cited in the following enumeration represent complete specimens, and for this reason it has been impossible to accurately determine much of the material. Many specimens consisted only of leaves, with no flowers or fruit, and such specimens, unless the material was especially characteristic or had the native name attached, were destroyed, the greater part of it being quite worthless for purposes of identification. 
No attempt was made to match specimens so as to reduce the total number, but each separate specimen reccived an individual number. 'There were no notes regarding the size of the plants, habitat, altitude above the sca, or whether wild or cultivated. Practically all the numbers represent shrubs or trees, with one or two herbaceous and several climbing plants.

Owing to the very unfortunate fact that the herbarium and botanical library at Manila was destroyed by fire in 1897 , before the American occupation, it was quite impossible to identify mucl of the material in Manila, and accordingly the trip mentioned above was planned in order to identify this botanical material and to take advantage of the many facilities afforded at the botanical garden at Buitenzorg.

At Buitenzorg there were rich collections from Borneo, Celebes, Moluccas, Java, Sumatra, the Malay Peninsula, and British India, but there was practically no Philippine material. Little trouble, however, was cxperienced in identifying those species of wide distribution. In the casc of the endemic species, the lack of authentic specimens for comparison was keenly felt, a fact that has had much influence on the description of new species in the present paper. Only five species are described as new in the following article, yet manifestly much of the material that I have placed in the several genera without specific names, is undescribed. It is true that many of the numbers so treated do not represent complete botanical specimens, yet some of them are in good condition and would afford a basis for full and accurate descriptions. In most cases, however, with the lack of authentic Plilippine material, it could not be ascertained with certainty whether or not the species had been described, and under the circumstances I have admitted such species without specific name.

The botanical material named below was collected mostly in the Islands of Luzon and Mindanao during the year 1901, and up to June, 1902. The greatest number of specimens from any one locality was obtained in Camarines Sur, Luzon, in the vicinity of Pasacao, some of the specimens being from that town, some from the barrio of Dalupaon, and some from a small river (Tinalmut) near Pasacao. As Dalupaon and Tinalmut are not given on the charts of the region, the specimens have all been credited to Pasacao. Other Luzon matcrial is from the vicinity of Manila, the Province of Tarlac, and the Provinces of Zambales and Benguet. The Mindanao material was chicfly collected in the district of Zamboanga, presumably from the vicinity of the town of Zamboanga, although some were collected at Tetuan and some in the Province of Surigao, probably from the vicinity of the town of Surigao, while a few specimens came from Placer. Several specimens are also from Mount Apo in the district of Davao. One or two specimens from Jolo and $\mathrm{Pa}-$ ragua, and considerable material from the Islands of 'Tinago, Bongao, and Dinagat are also included. 
When one considers that for nearly two years the work of the Forestry Bureau in Manila has been based almost entirely on the native names for the various tree species - a system of names extremely variable in Englishspeaking countries but much more variable and unreliable in the Philippines - the necessity for the present work is evident. Owing to the great number of different dialects spoken, a single species has often been found to have eight, ten, twelve, or even fifteen different recorded native names. To further complicate matters, the same name is often applied to totally unrelated species in various sections of the Archipelago. Therefore, in order to establish a firm basis for the study and classification of the Philippine forest trees, it is absolutely essential to first learn the proper scientific names in order that the published articles on the use and value of the many species may become available. Take, for example, the Tagalog word "Molave," the most common of the thirteen recorded native names. applied to the valuable timber tree Vitex littoralis Decne. To anyone outside of the Philippine Islands this name conveys no meaning, and even to one in the Philippines the native name alone would be of no value whatever if he wished to look up the notes and articles that have been published regarding this species and to ascertain whether or not it is to be found in the markets of the world, what are its common names, if any, in such markets, its value, uses, etc. With the scientific name once determined, however, all the notes regarding this timber tree that have been published in English, German, Dutch, French, or Spanish, at once become available.

The question of naming the greater part of the Philippine material would be a comparatively simple one if there existed in Manila a good botanical library and herbarium. Until such institutions are established, the greater part of the work on the Philippine flora must be done at the great botanical centers in Europe, especially at Kew, Berlin, Paris, and Leyden, for at the botanical institutions in these places is to be found practically all the Philippine material that has been collected in the past. In the United States the Philippine material is very limited. The plants of the Wilkes United States Exploring Expedition are to be found at the Gray Herbarium, the United States National Museum, and the New York Botanical Garden. A partial set of Cuming's Philippine collection is at the Gray Herbarium, a partial set of Haenke's material at the St. Louis Botanical Garden, and a set of A. Loher's more recent collection is in the United States National Herbarium. The collection of the Spanish Forestry Commission, which was destroyed by fire in 189\%, contained over 4,000 specimens of Philippine plants, collected by Señor Vidal, the chief of the commission, and named by him, with the collaboration of Mr. Rolfe of the royal gardens at Kew, lingland, a partial set of Cuming's Philippine material collected in the years 1836-1840, distributed by the British Museum, and many specimens from British India, the Malayan Archipelago, ete. To this botanical collection was added a very complete 
library, selected with special reference to the Philippine flora. The destruction of this herbarium and library, together with all the notes and records of the Forestry Commission, was a very great loss to Philippine botany, and it is imperative that a new herbarium and a new botanical library be established. The plants of the present enumeration form the basis of the new herbarium, and a beginning has been made on the library which already contains the leading works on the Philippine flora and many of the standard reference works.

The present status of the Philippine flora is rather peculiar. On one hand we have Blaneo's "Flora de Filipinas," with a large number of uncertain species or inidentifiable descriptions, and on the other hand we have Fernandez-Villar's "Novissima Appendix" to the third edition of Blanco's Flora, in which seores of species are credited to the Philippines which do not extend to the Archipelago, chiefly the species of British India. The genus Pandanus is a good illustration of the latter condition. Fernandez-Villar credits 17 species of Pandanus to the Philippines, while Dr. Warburg, a botanist who collected extensively in the Philippines, in his recent monograph of Pandanacea, ${ }^{1}$ eredits, and probably correctly so, but 14 species of the whole family to the Archipelago, several of which are there described as new, and 5 of Blanco's species considered as doubtful or at least unknown.' The same condition obtains also in such genera as Ficus, Eugenia, ete. In consideration of the fact that there are now eredited to the Philippines so many species that do not extend to the Archipelago, there is need of special care in identifications lest more species should be erroneously eredited to the Islands. No doubt errors will be found in the identifications in this paper, but in cases where there was much doubt concerning the identity of any particular specimen it has been entered without specific name, often with the related species noted, and this is especially the case where the related species has not been previously eredited to the Archipelago. 'J'he total number of specimens in the collection is 852 ; of these a considerable per cent were not in condition for accurate identification, and are not mentioned in the following paper, others are entered under their proper genera without specific names, when the material was in such condition that the genus conld be accurately deterınined. Species aceredited to the Philippines for the first time, regarding which especially careful identifications have been made, are in the following article preceded by an asterisk.

In the present enumeration there are represented 66 families, 225 genera, and about 400 species. Of these 400 species, 81 are presumably endemic to the Archiplago, 34 are reported from the Philippines for the first time, and 5 arc deseribed as new.

The sequence of families and genera is that adopted by Engler and Prantl in "Die Naturlichen Pflanzenfamilien."

${ }^{1}$ Engler's Pflanzenreich 3: 1900. 
Of the species here reported from the Philippines for the first time, nlany represent interesting, and, in some eases, peculiar eases of geographical distribution. The following species were previously known only from Celebes: Ficus minahassa Miq., Wallaceodendron celebica Koorder's, Gleditschia cclebica Konrders, Acetephila gigantifolia Koorders, Cratoxylon celebicum Blume, Strychnos celebica Koorders, Couthovia celebica Koorders, and Clerodendron minahassa Teysm. and Binn.

The following speeies were previously known only from the Moluecas: Casearia moluccana Blume, Lepiniopsis ternatensis Valeton, Clerodendron lanuginosum Blume, Ixora amboinica Decne, Albizzia littoralis, 'T. and $B$.

From Java: Luccosyke ulba Zoll. and Mor., Parinarium scabrum Aubl., Picrasma javanica Blume, Saurauia reinwardtiana Blume, Albizzia tomentella Miq., and Hydnocarpus heterophylla Blume, the latter also known from Sumatra.

From Borneo: Shorea scrobiculata Burck. Probably many other Philippine plants will be found in Borneo later when the flora is worked up. At present much of the Borneo material at Buitenzorg is undetermined.

From Burma, the Malayan Peninsula, and Sumatra: Pinus khasya Royle, Walsura robusta Roxb., and Erythroxylon burmanicum Griff.

Among the cases of geographical distribution of special interest must be noted the relatively large number of recently described Celebes species, which are here noted for the second time from the Southern Philippines; the discovery of Lepiniopsis ternatensis Valeton, in Mindanao; 4 distinct speeies of the genus Pinus from the mountains of Northern Luzon; and the discovery of Hydnocarpus heterophylla Blume, Paycna, leerii B. \& H. Erythroxylon burmanicum Griff., and Walsura robusta Roxb., in the Philippines.

One entire set of the material on which the following article is based is prescrved in the herbarium of the Forestry Bureau in Manila, and so far as duplicates were available one set deposited in the herbarium of the Botanical Institute at Buitenzorg and one set in the United States National Herbarium at Washington, all this duplicate material being distributed under the numbers cited in this article. 



\section{PLANT'A: AHERNIANE.}

[Enumeration of the species.]

\section{CONIFEREAS. \\ PINUS Linn.}

P. insularis Endl. Syn. Conif. 157. 1847.

Luzon, Prov. Benguet, 830.

Ilocano, Paruc; Igorrote, Al-al.

An endemic species also known from Lepanto and Nueva Ecija.

P. merkusii Jungh. and De Vriese, Pl. Nov. Ind. Bat. Or. 5. ८. 2.

Luzon, Prov. 'Zambales, 829.

This species is from the mountains of Zambales; also questionably reported from Pangasinan.

Borneo and Sumatra.

P. khasya Royle ex Gord. in Lond. Gard. Mag 16: 8. 1840.

Luzon, Prov. Benguet, 831.

Igorrote, Saleng.

Burma.

This species was credited to Luzon by F. Villar, Nov. App. 212, and evidently correctly so, as the specimen here referred to that species agrees very well with the published descriptions and figures of Royle's Pinus khasya. That this species should be found in Burma and again in Northern Luzon gives some cause for doubting theidentity of the Burma and Luzon specimens. Thisspecies is closely related to Pinus insuluris Findl.

P. sp. nov.?

Luzon, Prov. Benguet, 832, 833.

A species most distinct irom other Philippine material, especially in the cones which are much larger than in any of the above species, being $10 \mathrm{~cm}$. in length. No. 832 has an exceedingly rough cone. It was at first proposed to describe this species as new, but owing to the comparatively small amount of material, it has been considered advisable to wait until more material can be secured, and comparison made with authentic Asiatic inaterial.

Previously but two species of Pinus have been generally credited to the Philippines, although $\mathrm{F}$. Villar enumerates three, while in the present small collection under consideration we have four most distinct species. All these species are found in the mountainous regions in Nortbern Luzon, at altitudes above 1,000 meters. Possibly Pinus merkusii may later be found in the mountains of the southern part of the Archipelago, as its range outside of the Philippines extends much farther south than the province of Zambales, its only authentic locality in the in the Philippines. 


\section{( NETACEA.}

GNETUM Linn.

Gnetum gnemon Linn. Mant. 1: 125. 1771.

Mindanao, Prov. Surigao, 349, 410.

Malayan region.

\section{MARANTACEAE.}

DONAX Lour.

D. arundastrum Lour. Fl. Cochinch. 15. 1790. (Maranta arundinacea Blanco, Fl.

Filip. ed. 1, 7. 1837, non Linn.; M. dichotoma Wall. Cat. no. 6615. 1828.)

Mindanao, P'rov. 'Zamboanga, 654.

Southern Asia and the Malayan region.

\section{FAGACEAE.}

\section{QUERCUS Linn.}

Q. castellarnauiana Vidal, Rev. Pl. Vasc. Filip. 264. 1886.

Mindanao, Prov. Surigao, 527.

Endemic.

Q. philippinensis. A. DC. in DC. Prodr. 16:pt. 2, 97. 1868.

Mindanao, Prov. Surigao, 432.

Visayan, Uyyyan.

Wenzig ${ }^{1}$ considers that Quercus llanosii A. DC., Q. ovalis Blanco, Q. Blancoi A. DC., and Q. cyropode Miq., are all but forms of Q. philippinensis A. DC. Endernic.

\section{UIMACEAE.}

\section{CELTIS Linn.}

C. philippinensis Blanco, Fl. Filip. ed. 1, 197. 1837; Vidal, Sinopsis, t. $85 . f . A$. 1883.

Island of Dinagat, 466.

Endemic.

C. sp. indet.

Luzon, Prov. Camarines Sur, Pasacao, 7. Specimen imperfect, flowers wanting.

Not C. philippinensis Blanco.

\section{TREMA Lour.}

T. amboinensis Blune, Mus. Bot. Lugd. Bat. $2: 61.1856$.

Lizon, Prov. Tarlac, 707.

Himalayan region, Malayan Archipelago, etc.

\section{MORACEAE.}

\section{TAXOTROPHIS Blume.}

T. ilicifolia Vidal, Rev. Pl. Vasc. Filip. 249. 1886.

Luzon, Province of Camarines Sur, Pasacao, 14.

Mindanao, Prov. Zamboanga, 386.

Visayan, Cuyos-cuyos.

Endemic. 
PLATE I.

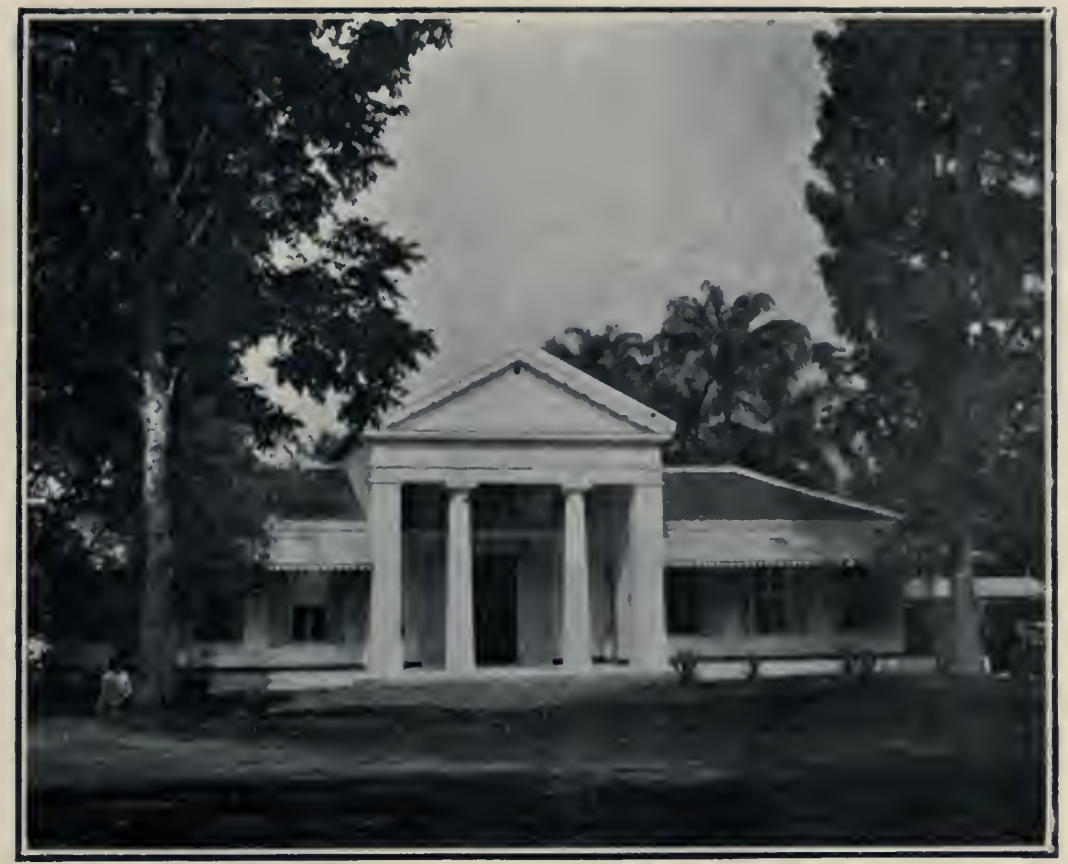

Fig. 1. -THE BOTANICAL MUSEUM.

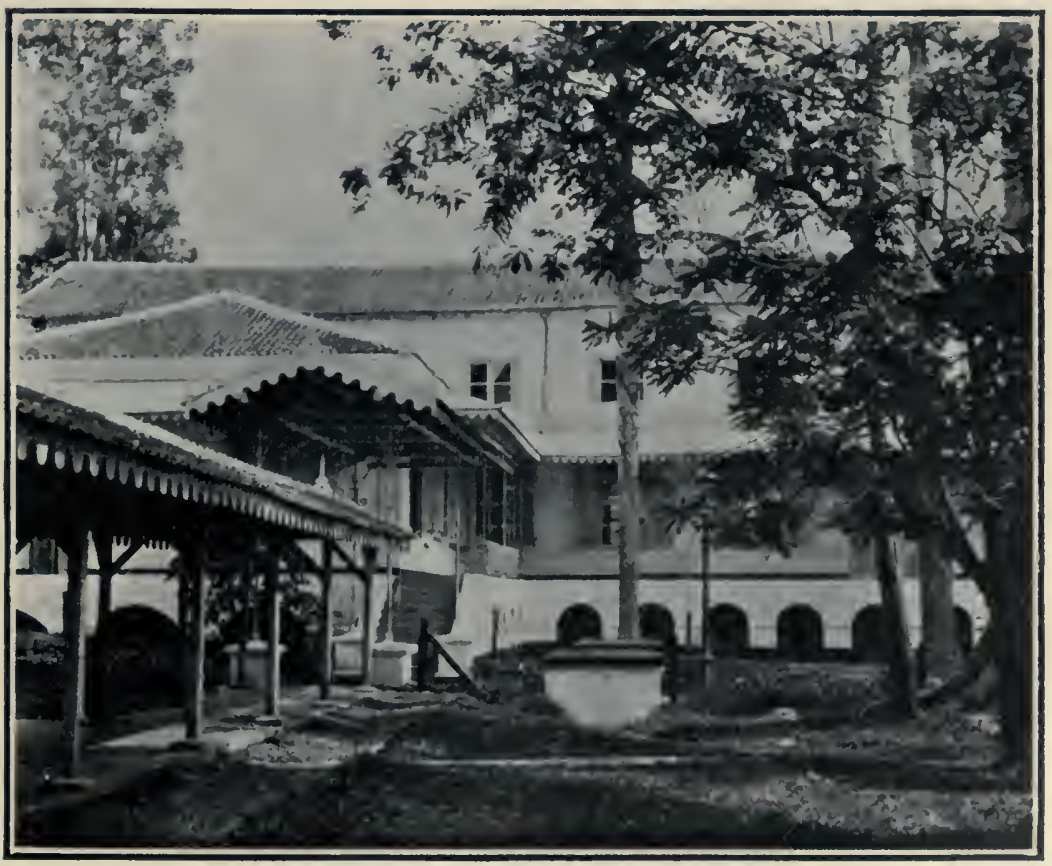

FIG. 2. -THE BOTANICAL MUSEUM, REAR VIEW. 

PLATE II.

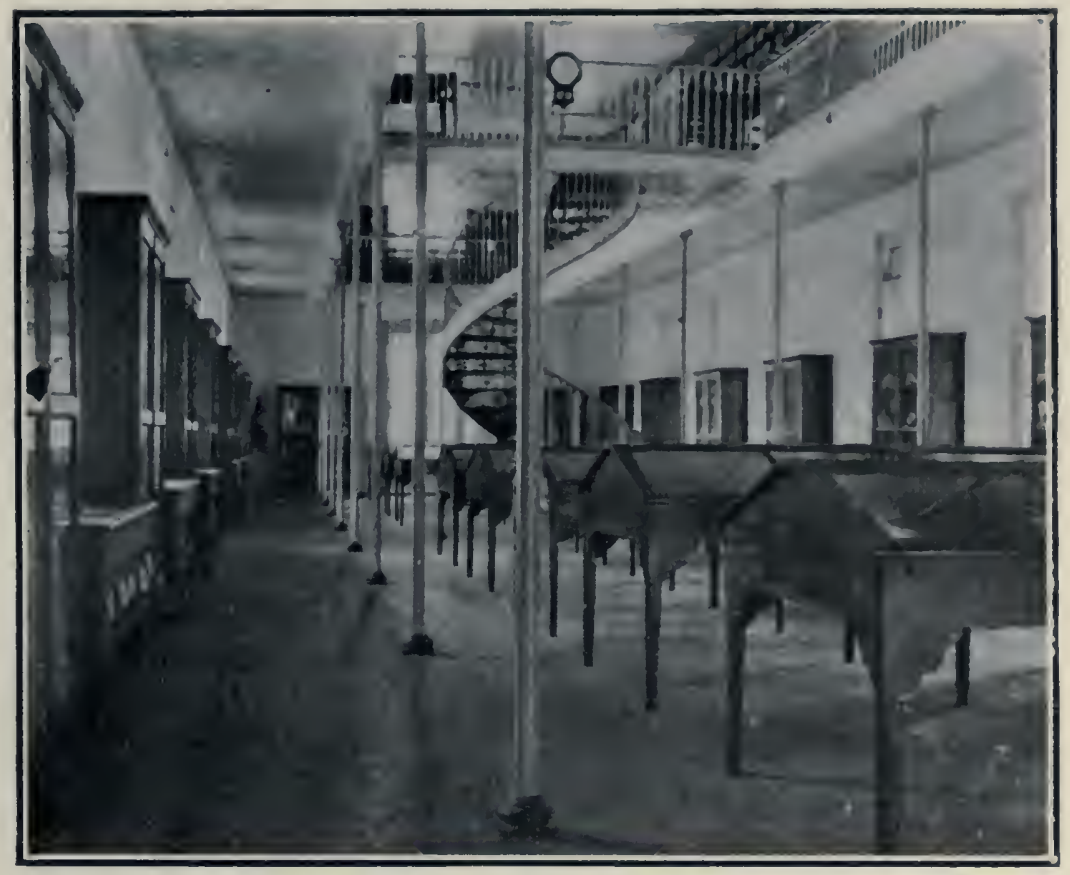

FIG. 1.-THE BOTANICAL MUSEUM, INTERIOR, MAIN FLOOR.

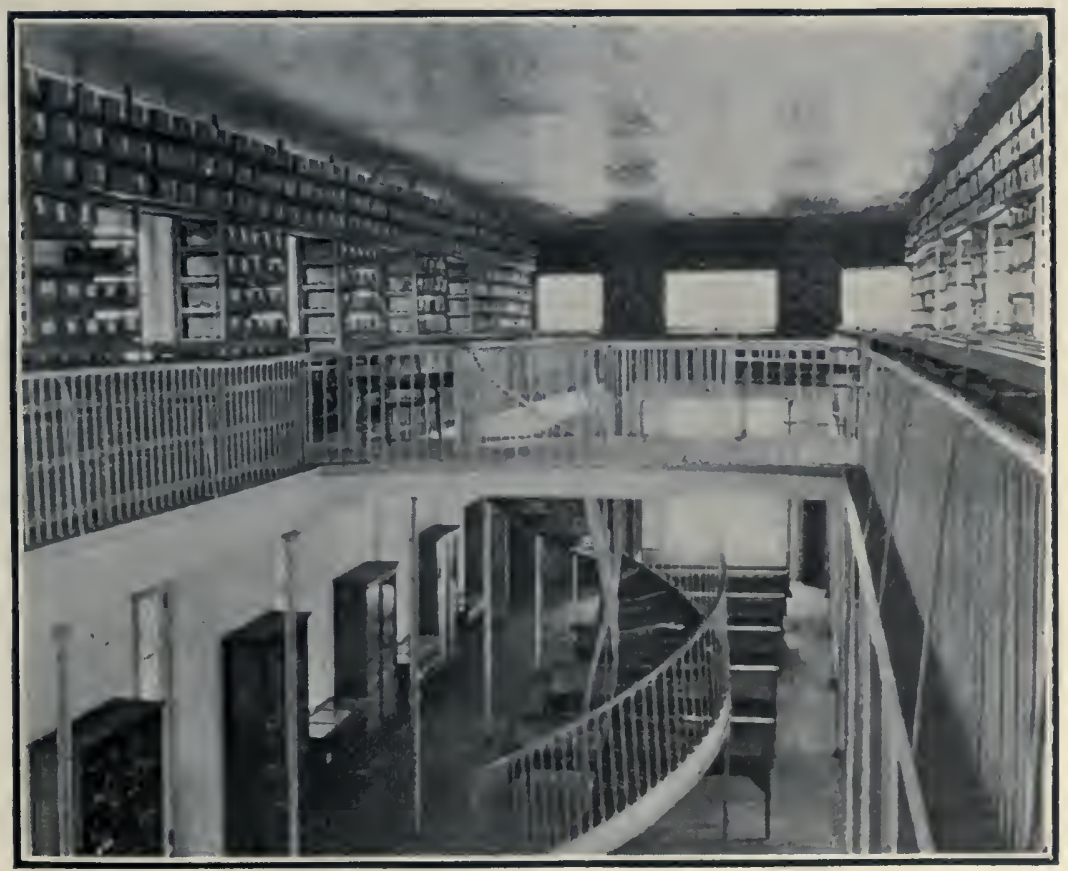

FIG. 2. -THE BOTANICAL MUSEUM, INTERIOR, FROM THE BALCONY. 

STREBLUS Iour.

S. asper Lour. Fl. Cochineh. 615. 1790.

Mindanan, 310 bis.

Widely distributed in sonthern Asia, Malayan Archipelago, etc.

\section{ARTOCARPUS Forst.}

A. blumei Trec. $A$ nn. Sc. Nat. III. 8: 111. 1847.

Luzon, Prov. Camarines Sur, Pasacao, 2.

Java.

A. cumingiana Trec. 1. c. 119. (A. oectu Blanco, Fl. Filip. ed. 1, 671, 1837 nonNoronl. 1790.)

Luzon, Prov. Camarines Sur, Pasacio, 119; Tarlac, 736; Prov. Bataan, Mariveles, 786; Mindanan, Prov. Surigao, 676.

Tagalog, Anubing.

Endemic.

A. incisa Linn. f. Suppl. 411.

Luzon, Prov. Bataan, Mariveles, 787; Mindanao, Prov. '/amuloanga, 605.

Tagalog, sntipols.

Widely distributed in sonthern Asia, Malayan Archipelago, etc.

A. integrifolia Jinn. f. 1. c. 412.

Mindanao, Prov. Surigao, 503.

Widely distributed.

A. sp. indet.

Luzon, Prov. Camarines Sur, Pasacao, 41, 281.

Material very imperfect.

Sect. Pelæomorphe.

FICUS Jinn.

F. pisifera Wall. Cat. no. 4504. 1828; King, Ann. Bot. Gard. Calcutta, 1: 3. pl. 1. 1888

Mindanao, Prov. '/amboanga, Tetuan, 544.

Malayan Peninsula and Archipelago.

Sect Urostigma.

F. pruniformis Blume, Bijdr. 451. 1825; King, l. c. $24 . \quad \%$. 21.

Luzon, Prov. Canarines Sur, Pasacao, 157.

Malayan Peninsula, Java, and Sumatra.

F. altissima Blume, I. c. 444. King, l. c. 30. pl. 30. (Urostigma altissima Mliq.; $F$. laccifera Roxb.)

Luzon, Prov. Camarines Sur, Pasacao, 172, 191, 798; Mindanao, Prov. Surigao, 509.

Himalayan region and Malayan Archipelago.

F. indica Linn. Sp. Pl. ed. 2, 1514. 1763, in part; King, l. c. $39 . \quad p l .45$.

Iuzon, Prov. Camarines Sur, Pasacao, 151, 194, 270, 296; Mindanao, Prov. Surigao, 664.

Burma to the Malayan Archipelago.

* F. indica Linn. var. gelderi (Miq.) King, l. c. 39. pl. 45. (Ficus gelderi Miq. Ann. Mus. Lugd. Bat. 3: 216, 287.)

Luzon, Prov. Camarines Sur, Pasacao, 23, 193.

Both numbers cited above have fruits larger than those described by Miquel and King.

Malayan Peninsula and Archipelago.

$6425-2$ 
F. clusioides Miq. Ann. Mus. Lugd. Bat. 3: 286. 1867; King, 1. c. $42 . \quad$ pl. 50.

Mindanao, Prov. Surigao, 407.

Endemic.

F. retusa Linn. Mant. 129. 1771; King, 1. c. $50 . \quad 1 l .61$.

Luzon, Prov. Camarines Sur, Pasacao, 176.

Sonthern Asia, Malayan Peninsula and Archipelago.

F. infectoria Roxb. Fl. Ind. 3: 550. 1832; King, J. c. $60 . \quad p l \quad 75$.

Luzon, Prov. Camarines Sur, Pasacao, 192.

India to Malayan region.

F. infectoria Roxl. var. caulocarpa (Miç.) King, 1. c. 63. pl. 79. (Urostigma (anlocarpum Miq. in Hook. Lond. Journ. Bot. 6:568. 1847.)

Mindanao, Prov. Surigao, 473.

Borneo.

* F. callosa Willd. Act. Acad. Berol. 102. 1. 4. 1798; King l. c. $64 . \quad p l .85$.

Luzon, Prov. Camarines Sur, Pasacao, 153; Mindanao, Prov. Zamboanga, 622.

India to Malayan region.

Sect. Sycidium.

F. quercifolia Roxb. Fl. Ind. 3: 534. 1832; King, l. c. 77 . pl. 95.

Luzon, Prov. Camarines Sur, Pasacao, 174, 239; Mindanao Prov. Surigao, 347, 388.

An extremely variable species of wide distribution, common about Manila.

F. heterophylla of Philippine authors, at least for most part, should doubtless be referred to this species.

Burma to Malayan Archipelago.

F. sp. indet. near $F$. melinocarpa Blume, Bijdr. 460; King 1. c. $94 . \quad p l .119$, from Java and Sumatra.

Mindanao, Prov. Surigao, Placer, 698. Specimen imperfect. .

* F. ampelas Burm. Fl. Ind. 226, 1768; King, 1. c. $90 . \quad p l$. 114.

lızon, Prov. Camarines Sur, Pasacao, 823; Mindanao, Prov. Zamboanga, Tetuan, 396.

Malayan Archipelago.

F. cumingii Miq. Lond. Journ. Bot. 7: 235. 1848. King, l. c. 92. pl. 118 .

Mindanao, Prov. Zamboanga, 629.

Endemic. A most distinct species.

Sect. Covellia.

* F. minahassæ Miı̣. Ann. Mus. Lugd. Bat. 3: 231, $296 . \quad$ 1867; King, l. c. $108 . \quad p l$. 140,141 .

Mindanao, Prov. Zamboanga, 494; Prov. Davao, 682.

Inoro (?) Matanug.

Both numbers eited above are without fruit, but in all other respects agree with authentic specimens of Miquel's species in Herb. Hort. Bot. Bog., and with King's description and plate. Previously known from North Celebes only.

F. leucantatoma I'oir. in Lam. Encycl. Suppl. 2: 654; King; l. e. 119. pl. $15 \%$. Luzon, Prov. Bataan, Mariveles 773; P'rov. Camarines Sur, Pasacao, 821.

Both numbers here referred to Ficus leucantatoma represent a narrow-leaved form.

Malayan A rchipelago.

F. glomerata Blanco, Fl. Filip. ed. 1, 683. 1837, non Roxb.

Iuzon, Prov. Bataan, Mariveles, 777. Mindanao, Prov. Surigao, 683, 305. 
These two numbers represent a very distinct species, not figured by King, and which are here referred to $F$. glomerata 13 lanco, a name invalidated by that of Roxburgh. Owing to the doubt which exists regarding Blanco's species, and the confused state of synonomy in the genus, no new name is here given to this form.

Ficus spp. indet., mostly of the section Urostigma. 392 and 606 bis, from Mindanao, represent a most distinct species, with exceeding large coriaceous leaves, not figured by King, and probably an undescribed species. No. 139 from Camarines Sur, is also a very distinet form, not figured by King. The remaining material is mostly very imperfect, and not possible for me to identify at this time. Nos. 5, 107, 238, 336, 350, 574, 631, and 809.

\section{URTICACEA.}

\section{PIPTURUS Wedd.}

P. asper Wedd. Ann. Sc. Nat. IV. 1: 197. 1854.

Iuzon, Manila, 712; Prov. Camarines Sur, l'asacao, 154; Mindanao, Prov. Surigao, 665; P’rov. Zamboanga, 619.

Endernic. Tagalog Dalonod.

\section{VILLEBRUNEA Gaudich.}

V. sp. indet., seems near V. frutescens Blume, Mus. Bot. Lugd. Bat. 2: 168.1856. Mindanao, Prov. Zamboanga 365; Prov: Davao, Mt. Apo, 685.

\section{LEUCOSYKE Mor.}

* L. alba Zoll. and Mor. in Syst. Verz. Ind. Archip. 100. 1842. Mindanao, Prov. Zamboanga, Tetuan, 361, 383.

Java.

L. capitellata Wedd, in DC. Prodr. 16: pt. 2, 23557. 1869.

Luzon, Prov. Camarines Sur, Pasacao, 113; Mindanao, Prov. Kamboanga, 617. Java.

\section{NYC'TAGINACEA.}

\section{PISONIA Jinn.}

P. umbellifera (Forst.) Seem. in Nadiaud. Fl. Tait. 46. (I'. excelsa Blume.)

Luzon, Prov. Bataan, Mariveles, 754; Prov. Camarines Sur, Pasacao, 171, 286; Mindanao, Prov. Surigao, 346.

Malayan region to Australia, etc.

\section{ANONACEA.}

\section{UVARIA Linn.}

* U. ovalifolia Blume, Fl. Jav. Anon. 27. 1828. Luzon, Prov. Camarines Sur, Pasacao, 186. Java.

U. sp. indet.

Luzon, Prov. Camarines Sur, Pasacao, 167.

The same as an unnamed specimen in Herb. Hort. Bot. Bog., from Celebes. 
CANANGA Hook. f. and Thoms.

C. odoratá (Lam.) Hook. f. and Thoms. Fl. Ind. 1: 130. 1855.

Mindanao, l'rov. Surigao, 455, 681.

Tagalog, Ilang-ilang.

Widely distributed in the Malayan Archipelago, Southern Asia etc.

ANAXAGOREA A. St. Hil.

A. luzonensis A. Gray, in Wilke's U. S. Expl. Exped. Bot. 1: 27. 1854.

Luzon, Prov. Camarines Sur, Pasacao, 181. Mindanao, Prov. Surigao, 357.

Visayan, liugang-nso.

Malayan region ete.

\section{UNONA Linn, f.}

U. discolor Vahl. Symb. Bot. 2: 63. 1. 36.1798.

Luzon, Manila, 730 .

A form very near this species and so referred.

Tropical Asia and the Malayan region.

PH两ANTHUS Hook. $i$. and Thoms.

P. cumingii Miq. Fl. Ind. Bat. 1: pt. 2, 51. 1855.

Lizzon, Manila, 742.

Endenic.

MITREPHORA Hook. f. and Thoms.

M. reticulata Hook. f. aml Thoms. in Hook. Fl. Brit. Ind. 1: 77. 1875.

Juzon, Prov. Bataan, Mariveles, 745.

Malayan Peninsula and Arehipelago.

XYLOPIA linn.

X. DEHISCENS (Blanco). (Unona dehiseens Blanco, Fl. Filip. ed. 1, 466. 1837; Nylupure lulencoi Vidal, Sinopsis, 1. 5. f. (: 188.3.)

luzon, Prov. Camarines Sur, Pasacao, 25, 125, 136.

Tagalog, Lmintur.

Endenic.

ANONA linn.

A. muricata Jinn. Sp. Pl. 536. 1753.

Mindanao, Prov. Surigao, 661.

Introduced from tropical Anerica.

\section{MYRISTICACEA.}

G. sp. indet.

\section{GYMNACRANTHERA Warb.}

Mindanao, Prov. Zamboanga, Tetuan, 369, material very imperfect.

\section{MYRISTICA Iinn.}

M. philippinensis Lam. Act. Paris. 161. 1788; Encycl. 4:387. 1797; Warburg, Monog. Myrist. 386. 1. 12. f. 1-8, 1897. (M. Brarteate A. DC. in DC. Prodr.

14: 192. 1847; M. Luzonica Blanco, Fl. Filip. ed. 1, 664. 1837.)

Mindanao, I'rov. '/amboanga, Tetuan, 558.

Endemic; cultivated in Mauritius, Calcutta, Brazil, etc.

M. simiarum A. DC. in DC. Prodr. 14: 191. 1847; Warburg, 1. c. 397.

Mindanno, Prov. Zamboanga, Tetuan, 364, 573.

Endemic. 
M. guatteriifolia A. DC. Ann. Sc. Nat. IV. 4: 20. 1. 4. 1855; Prodr. I. c., 193; Warlurg, 1. c., 412. 1. 19. f. 1-4.

I.uzon, Prov. Camarines Sur, Pasacan, 20; Mindanao, Prow. Yamboanga Tetuan, 590.

Philippine Islands, also Island of Lahuan north coast of Borneo.

M. cumingii Warb. Monog. Myrist. 442. 1. 1s. 1897. (M. iners I3lume, in A. DC. 1. c. 190. pp.)

Mindanao, Prov. Surigao, 421. La1zon, Prov. Bataan, Mariveles, 758.

Tagalog, Malatalung.

Endemic.

M. mindanænsis Warb. 1. c. 497 . เ, 13.

Mindanao, Prov. Zamboanga, Tetuan, 393, 589.

Endemic.

M. sp. indet.

Mindanao, Prov. Zamboanga, 363. Material too imperfect for accurate identification.

\section{MONIMIACEA.}

\section{KIBARA Endl.}

K. coriacea (Blume) Perk. in Engler's Bot. Jahrb. 25: 575.1898.

Mindanao, Prov. Zamboanga, 371, 850.

Java and Celebes.

In the recent monograph by Perkins and (rilg." no species of this family is credited to the Philippines, although this species is reported by Vidal, No. 950. The specimens here referred to $k$. corincea differ somewhat from the description of Perkin's and Gilg.

\section{LAURACEAS.}

\section{CINNAMOMUM Blume.}

C. mercadoi Vidal, Rev. Pl. Vasc. Filip. 224. 1886. (C. iners F. Vill. Nov. App. 180, non Reinw.)

Luzon, Prov. Camarines Sur, Pasacio, 50.

Endemic.

C. sp. indet.

Mindanao, Prov. Zamboanga, 647.

\section{PHOEBE Nees.}

P. sp. indet. Seems to be $P$. umbelliflor Blume, but the material is very imperfect. Luzon, Prov. Camarines Sur, Pasacao, 127.

D. sp. inclet.

\section{DEHAASIA Blume.}

Lizon, Prov. Camarines Sur, Pasacao, 130. Material very imperfeet.

\section{HERNANDIACEAE.}

\section{HERNANDIA Linn.}

H. peltata Meissn. in DC. Prodr. 15: pt. 1, 263. '1864.

Luzon, Prov. Camarines Sur, Pasacao, 17; Mindanao, Prov. Surigao, 409, 458. Southern Asia, Malayan region, etc.

\footnotetext{
'Engler's P'lanzen reich, 4: 1901.
} 


\section{MORINGACEA.}

MORINGA Juss.

M. oleifera Lam. Fincyel. 1: 398. 1783. (1\%. pterygosperma Gaertn. Fruct. 2: 314. 1791.)

Lizzon, Prov. Bataan, Mariveles, 768.

Tagalog, Mfuhungry.

Asia.

\section{PI'T'TOSPORACEA:}

PITTOSPORUM Banks.

P. sp. indet. Closely related to $P$. ferruginenm Dry., which extends from the Malayan Peninsula and Archipelago to Australia, but differs in certain characters.

Minclanao, Prov. Zamboanga, Tetuan, 373.

\section{ROSACEA:}

\section{PYGEUM Gaertn.}

P. latifolıum Miq. Fl. Ind. Bat. 1: pt. 1. 361. 1855.

Mindanao, Prov. Zamboanga, Tetuan, 367.

Malayan Archipelago.

PARINARIUM Aubl.

* P. scabrum Hassk. Flora, 27: 585.1844.

Mindanao, Prov. Surigao, 375.

A Javanese species, which No. 375 resembles very closely, and to which it is referred, differing in some slight characters.

\section{CONNARACEA.}

\section{CONNARUS Linn.}

C. trifoliatus (Turcz.) Rolfe, in Vidal, Rev. 11. Vasc. Fỉlip. 103. 1886. (Ani8nstemon trifolintus Turez. Bull. Soc. Nat. Mosc. 20: pt. 1, 152. 1847; C. Rolfei Vidal P'lanerog. Cuming. 23, 106. 1885.)

Luzon, Prov. Camarines Sur, Pasacao, 218.

Findemic.

\section{LEGUMINOSAS.}

GLIRICIDIA 11. B. K.

G. maculata II. 13. K. Nov. Gen. et Sp. I'l. 6: 393. 1823.

Isuzon, Prov. Bataan, Balagna, 309.

A species introduced from Central America and now widely distributed in the Philippines commonly known as mulre encuo.

\section{SERIANTHES Benth.}

S. grandiflora (Wall.) Benth. in Hook. Lond. Journ. Bot. 3: 225. 1844. (Inga grundiflore Wall.)

Island of Dinagat. 447 .

Throughout the Malavan region. 


\section{PITHECOLOBIUM Mart.}

P. dulce (Willd.) Benth. 1. c. 199. (Inga dulcis W'illd.)

Luzon, Prov. Bataan, Mariveles, 763.

Tagalog, Cumenchiles.

A native of tropical America, now widely distributed in the Plitippines.

P. lobatum (Grah.) Benth. 1. c. 208. (Ingu lobalu Giral.)

Mindanao, P'rov. Zamboanga, Tetuan, 555.

Malayan Peninsula and Archipelago.

P. acle (Blanco:) Vidal, Rev. l'l. Vasc. Filip. 121. 1886. (Mimora acle Blanco, Fl. Filip. ed. 1, 738. 1837.)

Luzon, Prov. Camarines Sur, Pasacao, 74, 142.

Tagalog, Acle.

Endemic.

WALLACEODENDRON Koorders.

* W. celebica Koorders, Mededeel.'s Lands. Plant. 19: 630. 1898.

Luzon, Prov. Camarines Sur, Pasacao, 49 bis.

A monotypic genus, previously known only from the original collection at Minehassa, Northeast Celebes.

\section{ALBIZZIA Duraz.}

A. lebbek. (Willd.) Benth. in Hook. Lond. Journ. Bot. 3: 87. 1844.

Luzon, Manila, 741, 721.

Widely distributed in the Tropies.

*A. littoralis Teysm. and Binn. Tijlschr. Ned. Ind. 29: 259.1866.

Luzon, Prov. Camarines Sur, Pasacao, 69.

A species extending from Java to the Moluceas and Celebes.

A. procera (Willd.) Benth. 1. c. 89. (Acucia procera Willd.)

Luzon, Prov. Bataan, Balagna, 601, 846.

Tagalog, Alalangat.

Widely distributed in the Malayan region.

A. Iucida (Roxb.) Benth. 1. c. 86. (Mimosu lucida Roxb.)

Mindanao, Prov. Surigao, 678.

Southern Asia and the Malayan region.

*A. tomentella Miq. Fl. Ind. Bat. 1: pt. 1, 20. 1855.

Mindanao, Prov. Surigao, 395. Previously known from Java.

A. sp. indet. near A. julibussen Durazz., but the material is very imperfect.

Luzon, Manila, 743.

PARKIA R. Br.

P. roxburghii G. Don. Gen. Syst. 2: 397. 1834.

Juzon, Tarlac, 702.

Tagalog, Cupang.

Tropical Asia, Malayan region, etc.

SINDORA Miq.

S. wallichii Bentl. in Hook. Icones, t. 1017-18.

Island of Dinagat, 439.

Singapore and Malacea. 


\section{TAMARINDUS Linn.}

T. indica Limn. Sp. I'l. 34. 1753.

Mindanan, Prov. Surigao, 315; Pror. Zamboanga, 446.

Tagalog, Stampaioc.

A common species about town; cultivaterl. Tropieal $\Lambda$ sia, $\Lambda$ frica ete.

AFZELIA smith.

A. bijuga (1)C.) A. (iray, in Wilke's U. S. Expl. Expecl. Bot. 1: thiT. 1.51. 1854. (Outen lijuga DC.)

lazon, Prov. Camarines Sur, l'asarao, 208.

Tagalog, Ipil.

Distributed thronghout the Malayan region, to Australia. A valuable timber tree.

A. rhomboidia Viclal, Phanerog. Cuming. 110. 1885.

Luzon, Prov. Camarines Sur, Pasacao, 146. Island of Culion, 704.

Endemic: A valuable timber tree. Tagalog, Tindulo.

\section{CASSIA Linn.}

C. florida Vahl. Symb). But. $3: 57.1794$.

Iuzon, Manila, ill.

Malayan region, etc.

\section{GLEDITSCHIA Scop.}

${ }^{*}$ G. celebica Koorders, Mededeel. 's Lands Plant. 19: 435. 1898.

Iuzon, Prov. Camarines Sur, Pasacao, 62.

A species previously known only by the original collection from North Celebem. Related to $G$. rolfei Vidal, but quite distinct.

\section{C 无SALPINIA Linn.}

C. pulcherrima (Linn.) Sw. Obs. 166. 1791. (Poinciana pulcherrimu Linn.)

Mindanao, Prov. Surigao, 521.

Usually found about towns. Introdnced.

'Tropics of both hemispheres, native country not clearly kuown.

C. sappan Linn. Sp. Il, 381. 1753.

Lu\%on, Prov. Bataan, Mariveles, 771.

Tagalog, Nilucuo.

Tropical Asia, Malayan region.

\section{SOPHORA Jinu.}

S. tomentosa Liun. Sp. Pl. 373. 1753.

Luzon, P'rov. Camarines Sur, Pasacao, 213.

A species found near the seacoast in the Tropica of both hemispheres.

\section{INDIGOFERA Linn.}

I. teysmanni Miq. Fl. Ind. Bat. 1: pt. 1, 1083. 1855.

Luzon, Prov. Camarines Sur, Pasacao, 234, 2:5. Mindanao, Prov. Surigao, 434.

Malayan Archipelago.

I. tinctoria Linn. Sp. Pl. 751. 1753.

Luzon, Prox: Camarines Sur, P'asacao, 227.

Widely distributed in the Tropics and cultivated for indigo. 


\section{DESMODIUM I)esv.}

D. umbellatum (Linn.) DC. Prodr. 2: 325. 1825. (Hedlysarum renlsellulum Linn.)

Luzon, Prov. Camarines Sur, Pasacao, 222; Mindanao, Prov. Surigao, 408.

Seychelles, southern Asia, Malayan Archipelago etc., to Australia,

DALBERGIA Linn. $\mathrm{f}$.

D. ferruginea Roxb. Hort. Beng., 98, 1814.

Mindanao, Prov. Zamboanga, 632.

Southern Asia, Malayan region.

1). ferrugineu Roxb., is cited by Baker in Hook. f. Fl. Brit. Ind. 2 : 237, as a synonym of $D$. stipulacen Roxb., but is here held a distinct species.

PONGAMIA, Vent.

P. glabra Vent. Jard. Nalm. 28. 1. 28. 1803.

Luzon, Prov. Camarines Sur, Pasacao, 46, 206. Mindanao, Prov. Surigao, 496; Prov. Zamboanga, Tetuan, 581; Island of Tinago, 420.

A species found near the seacoasts from the Seychelles to Australia.

ERYTHRINA Linn.

E. indicá Lam. Encycl. 2: 391. 1786.

Iuzon, Prov. Camarines Sur, Pasacao, 30; Mindanao, Prov. Davao, Mt. Apo, 675 .

From British India, through the Malayan region to Australia.

\section{OXALIDACEA.}

\section{AVERRHOA Linn.}

A. carambola Linn. Sp. Pl. 428. 1753.

Mindanao, Prov. Surigao, 522.

This species, as well as $A$. Unlimbi Linn., is commonly cultivated about towns in the Philippines, and is widely distributed in the Tropies.

\section{ERYTHROXYLACEA.}

\section{ERYTHROXYLON Linn.}

* E. burmanicum Griff. Notul. 4: 468, 1849.

Luzon, Prov. Camarines Sur, Pasacao, 12, 292.

Visayan, Manambo.

Burma, Malayan Peninsula, and Sumatra.

\section{RU'TACEX:}

\section{EVODIA Forst.}

EVODIA MINDAN ÆENSIS Merrill, sp. nov.

A shrub or small tree with axillary inflorescence and thin, membranous, palegreen, trifoliate leaves. Young branches terete, densely puberulent. Petioles 10 to $11 \mathrm{~cm}$. long, opposite, pubescent; leaflets very thin, nearly sessile, ovate, short acuminate, the rounded base slightly cordate, glabrous above except on the pubescent mid-nerve, pubescent on the nerves beneath, 12 to $20 \mathrm{~cm}$. 
long, 8 to $10 \mathrm{~cm}$. wide, lateral nerves about 14 pairs, rather prominent, anastomosing near the margin. Panicles narrow, about equaling the petioles, the primary branches 1 to $2 \mathrm{~cm}$. long, densely pubescent. Flowers small, on short (1-2 mm.) puberulent pedicels. Sepals 4 , rounded, pubescent, about $1 \mathrm{~mm}$. long. P'etals 4, about $2.5 \mathrm{~mm}$. long, ovate, acute, punctate. Stamens glabrous, exceeding the petals. Ovary hirsute. Carpels 4 , about $3 \mathrm{~mm}$. long, sparingly pubescent.

Type specimen collected at Tetuan, Prov: of 'amboanga, Mindanao, No. 592, also 301 from Surigao, Mindanao, is referred here. A species characterized by its rather large, very thin pale-green leaves. No. 301 is in fruit, and is placed here as it agrees with the type in all characters of inflorescence and vegetation, except that the leaves are somewhat sinaller, not exceeding 12 cin. in length.

Visayan, Bintoco.

E. sp. indet.

Very near Evodia minahassx Koorders, from North Celelses, but possibly distinet. Specimen with fruit only.

Mindanao, l'rov. Surigao, 435.

E. sp. indet.

Material very imperfect.

Luzon, Prov. Camarines Sur, Pasacao, 128.

\section{LUNASIA Blaneo.}

L. amara Blanco, Fl. Filip. ed. 1, 783. 1837.

Mindanao, Prov. Zamboanga, Tetuan, 559, 643.

Endemic.

\section{GLYCOSMIS Correa.}

G. pentaphylla Correa, Ann. Mus. Par. 6:386. 1805.

Luzon, Manila, 725.

From tropical $\Lambda$ sia to Australia.

\section{MICROMELUM Blume.}

M. tephrocarpum Turez. Bull. Soc. Nat. Mosc. 31 : pt. 1, 379. 1858. (M. gla(rescens F. Vill. Nov. App. 36, non Benth.)

Mindanao, Prov. Surigao, Placer, 380, 401; Prov. '/amboanga, Tetuan, 381. Celebes.

M. molle Turez. 1. c. 380 ?

Mindanao, l'rov. '/amboanga, 368.

Endemic.

M. sp. indet.

Minlanao, Prov: '/amboanga, 591, 624.

CLAUSENA Burm.

C. excavata Burm. Fl. Ind. 87. 1. $2 \%$. 1768.

Luzon, Prov. Camarines Sur, Pasacao, 49.

Southern $A$ sia and the Malayan region.

TRIPHASIA Lour.

T. aurantiola Lour. Fl. Cochinch. 15\%. 1790.

Mindanao, Prov. Surigao, 524.

Tropical Asia and the Malayan region. 


\section{CITRUS Linn.}

C. hystrix J)C. Cat. Ilort. Monsp. $\mathbf{9 7 .} 1813$.

Luzon, Prov. Camarines Sur, Pasacao, 91; Mindanao, P'rov. Surigan, 51?.

Southern Asia and the Malayan region.

C. $\mathrm{sp}$ indet.

Mindanao, Prov. Surigao, 366.

\section{SIMARUBACEAE.}

BRUCEA J. F. Mill.

B. sumatrana Roxb. Hort. Beng. 12. 1814.

Mindanao, Prov. Surigao, 512.

Tropical Asia to Australia.

\section{PICRASMA Blume.}

*P. javanica Blume, Bijdr. 248. 1825.

Mindanao, Prov. Surigao, Placer, 696; Prov. Zamboanga, Tetuan, 557.

Java.

\section{AILANTHUS Desf.}

A. moluccana DC. Prodr. 2 : 89. 1825.

Luzon, P'rov. Camarines Sur, Pakacao, 93.

No. 93 differs somewhat from this species, especially in the fruit, but is very close to A. moluccana, and is so referred.

Moluccas.

\section{BURSERACEA:.}

\section{CANARIUM Linn.}

C. cumingii Engl. in DC. Monog. Phanerog. 4 : 132. 1883.

Mindanato, Prov. Surigao, 697.

Endemic.

C. commune Linn. Mant. 1 : 127.1771.

Mindanao, Prov. Surigao, 334.

From India to the Malayan Peninsula, Java to North Celebes. No. 334 seems to agree very well with this species. Conarium comune Blanco, non Linn., is C. cumingii Engl.

C. sp. indet. Related to Canarium legitimum Miq., from Amboina, but quite distinct.

Iuzon, Prov. Camarines Sur, Pasacao, 117; Prov. Bataan, 789.

C. sp. indet.

Isuzon, Prov. Camarines Sur, Pasacao, 63, 134, 788.

\section{MELIACEAS.}

TOONA M. Roem.

Toona ciliata Roem. Syn. Hesper. 139. 1846. (Cedrela toona Roxb. ex Rottl. in Ges. Naturf. Fr. Neue Schr. 2: 198. 1803.)

Luzon, Prov. Camarines Sur, Pasacao, 73.

British India, Malayan Peninsula and Archipelago. 
XYLOCARPUS K゙on.

*X. obovatus (Blume) A. Juss. Mem. Mus. Paris, $19: 244.1830 . \quad$ ( ('urapu oborula IBlume, Bijclr. 1:179. 1825.)

Luzon, Prov. Camarines Sur, Pasacao, 268; Mindanao, Prov. Surigao, 505; Island of Dinagat, $80,483$.

Widely distributed along the seashores in the Tropies of the Old Worll. 1. Firmutum Koen., is also found in the Philippines.

\section{SANDORICUM Cav:}

S. indicum Cav. Diss. 7: 359. t. 202, 203. 1801.

Mindanao, I'rov. Zaunboanga, Tetuan, 459.

Tropical Asia and the Malayan region.

S. sp. indet.

Luzon, Prov. Camarines Sur, Pasacao, 228. "A species not found in Java nor in Northeast Celehes." -Koorders.

D. sp. indet.

\section{DYSOXYLUM Blume.}

Very near, and perhaps identical with $D$. arborescens Miq., which extends from Java and Sumatra to North Celebes, but the material is very imperfect.

Luzon, Prov. Camarines Sur, Pasacao, 813.

D. sp. indet.

Mindanao, Prov. Surigao, 642.

\section{CHISOCHETON Blume.}

C. tetrapetalus Turez. Bull. Soc. Nat. Mosc. 1: 411.1858.

Luzon, Prov. Bataan, Mariveles, 761; Prov. Camarines Sur, Pasacao, 9; Mindanao, Prov. Surigao, Placer, 406.

Java.

C. sp. indet. Mindanao, Prov. Surigao, 533, 585. Material very imperfect.

AMOORA. Roxb.

A. sp. indet. Apparently identical with an unnamed specimen, No. 12328, Teysmann, from Celebes in Herb. Hort. Bot. Bog.

Mindanao, Prov. Surigao, 324.

AGLAIA Lour.

A. sp. indet., seems very near $A$. gunggo Miq., from Sumatra.

Island of Dinagat. 481.

A. sp. inclet., in some respects very elose to .1. elepticu Blume, from the Malayan region.

Island of Tinago, 422; Mindanao, Prov. Zamboanga, 638.

A. Ep. indet. The same as an unnamed specimen, No. 12338. Teysmann, in IIerb. Hort. Bot. Bong., from Celebes.

Mindanao, Prov. 'aumboanga, 535, 563.

A. sp. indet. A very distinct form, in fruit only.

Litzon, I'rov. Camarines Sur, P'asacao, 18.

"Neither in Java nor in Northeast Celehes." - Koorders.

A. sp\}. indet. $8,123,245,247,415,487,536,551,667$. 
WALSURA Roxb.

*W. robusta Roxb. Hort. Beng. 32. 1814.

Luzon, Prov. Camarines Sur, Pasacao, 108, 264.

A species extending from the Hinalayan region to Burma, and the Andaman Islands.

Some hesitation was felt in referring the l'hilippine specimens to this species, the only one in the section (Survalu), although in all but one or two minor details the inaterial here referred to Wulsura robustn agrees very well with the published descriptions and with the Asiatic material so named, in Herb. Hort. Bot. Bog.

\section{MALPHIGIACEAE.}

TRISTILLATEIA Thou.

T. australasica A. Rich. Sert. Astrolab. 38. t. 3i. 1834.

Luzon, Camarines Sur, Pasacao, 18;.

Visayan, Bagmit.

Malacea, Malayan Archipelago to Australia.

\section{EUPHORBIACEA:}

\section{ACTEPHILA Blume.}

*A. gigantifolia Koorders, Mededeel 's Lands Plant. 19: 625. 1898.

Mindanao, Prov. Surigao, 690.

A species previously known only from the type collection, northeast Celebes.

\section{PHYLLANTHUS Linn.}

P. distichus (Linn.) Muell. Arg. in DC. Prodr. 15: pt. 2, $413.1866 . \quad$ (Cissa distichn Limn.)

Luzon, Manila, 709.

Tagalog, Bunguiling or 1 bee.

Tropical Asia, the Malayan region, ete.

P. reticulatus Poir. in Lam. Encycl. 5: 298. 1804.

Luzon, Manila, 726; Prov. Camarines Sur, Pasacao, 197, 803 bis; Mindanao,

Prov. 'Zamboanga, 'Tetuan, 560.

Widely distributed in the Tropics.

\section{GLOCHIDION Forst.}

G. cumingii Muell, Arg. Linnæea, 32: 61. 1863.

Luzon, Prov. Camarines Sur, Pasacao, 149, 271.

Fndemic.

G. DIVERSIFOLIUM (Miq.) Phyllanthus diversifolius Miq. Fl. Ind. Bat. Suppl. 448. 1861.

Luzon, I'rov. Camarines Sur, Pasacao, 161, 274; Mindanao, Prov. 'Zamboanga, 616.

Java and Sumatra.

G. molle Blume, Bijdr. 586. 1825. (Phyllenthus mollis Muell. Arg.)

Luzon, Prov. Camarines Sur, Pasacao, 173.

Java to Celebes. 
*G. littorale Blıme, Bijdr. 585. 1825. (Phyllanthus littoralis Muell. Arg.)

Mindanao, l'rov. Surigao, 402, 502, 526.

Malayan Peninsula and Java.

G. sp. indet. Near C. arborescens Blume, from Java, but distinet.

Mindanao, I'rov. Surigao, 460.

G. spp. indet. Owing to imperfect material, and lack of material for comparison, many species in this dificult genus have not been determined, Nos. 160, 162, $185,202,280,323,342,343,351,474,825$.

\section{SAUROPUS Blume.}

SAUROPUS ANDROGYNUS (Linn.): Chuytia androgyna Linn. Mant. 1: 128. 1767. Suuropus albicans Blume, Bijdr. 596. 1825.

Imzon, Prov. Camarines Sur, Pasacao, 288.

India and the Malayan region.

C. sp. indet.

\section{CYCLOSTEMON Blume.}

Luzon, Prov. Camarines Sur, Pasacao, 11.

Flowers only. No. 182 is also questionably referred to this genus.

\section{BACCAUREA Lour.}

B. tetrandra (Baill.) Muell. Arg. in 1)C. Prodr. 15: 465. 1866. (Adenocrepis tetrundra Baill.)

Luzon, Prov. Bataan, Mariveles, 760 .

Endemic.

\section{ANTIDESMA Linn.}

A. ghæsembilla Gaertn. Fruct. 1: 189. 1 . 39. 1788.

Lu\%on, l’rov. Camarines Sur, Pasacan, 188, 796, Mindanao, Prov. Zamboanga, Tetuan, 612.

Africa to the Malayan region and Australia.

A. sp. indet. Seems very near to a sterile specimen from North Celehes, No. 16803 Herbarium Koordersianum in Herb. Hort. Bot. Bog., also somewhat resembles A. Innceolatum Tul.

Luzon, I'rov. Camarines Sur, Pasacao 817. Specimen in fruit.

A. sp. indet. Very near Antidesur "No. 2" Koorders. Nededeel 's Lands P'lant.

19: 58 . No. 16854 Kuorders, from North Celeles.

Tinago Island, No. 418, specimen in fruit.

\section{BRIDELIA Willd.}

B. ovata Decne. Nouv. Ann. Mus. Par. 3: 484. 1835.

Mindlanao, Prov. Zambonga, 372, 641.

Malayan Peninsula and Archipelago to Australia.

\section{CROTON Linn.}

C. leiophyllus Muell. Arg. Linnee, 34 : 103. 1865-66.

Mindanao, Prov. Zamboanga, Tetuan, 571.

A species elosely related to C. levifolins Blume.

Endemic.

C. sp. indet.

Luzon, Prov. Camarines Sur, Pazacao, 265. 
CLAOXYLON A. Juss.

C. sp. indet. Very near C. rulescens Miq.

Luzon, Prov. Camarines Sur, Pastacao, 266, 293.

C. sp. indet. Related to C. longifolium Muell. Arg., and may be identical with this species, but the material is too poor for accurate identification.

Mindanao, Prov. '/amboanga, 556.

C. sp. indet. May be referable to Clusiylon indicum Hassk.

Mindanao, Prov. Surigao, 499.

Visayan, Sieguey.

\section{MALLOTUS Lour.}

M. moluccanus (Linn.) Muell. Arg. Linniea, 34: 185. 1865-66. (Croton moluccrmus Linn.)

Luzon, Manila, 715 ; Prov. Camarines Sur, Pasacau, 48, 260. Mindanao, 1'rov. Zamboanga, 394 .

Malayan region.

M. paniculatus (Lam.) Muell. Arg. l. c. 189. (Croton paniculitus Laur.)

Luzon, Prov. Cảmarines Sur, Pasacao, 87, 232.

Malayan Peninsula and Archipelago, ete.

M. philippinensis (Lam.) Mnell. Arg. 1. c. 192. (Croton philippinensis Lam.)

Mindanao, Pros: Zamboanga, 633.

India, Malayan region to Australıa.

M. ricinoides (Pers.) Muell. Arg. l. c. 195. (Croton ricinoides Pers.)

Luzon, Prov. Camarines Sur, Pasacao, 326. Mindanao, Prov. Zamboanga, 578;

Prov. Davao, 674.

Southern Asia, Malayan region, ete.

M. sp. indet. Near M. plutufuirii Hemsley. Material very imperfect.

Luzon, Manila, 732; Prov. Camarines Sur, Pasacao, 412.

ALCHORNIA Swartz.

A. javensis Muell. Arg. Linnaea, 34: 170. 1865-66.

Mindanao, Prov. '/amboanga, 589, 620, 649.

Java.

CLEIDION Blume.

*C. javanicum Blume, Bijdr., 613. 1825.

In lia, Malayan Arehipelago, etc.

Luzon, Prov. Camarines Sur, Pasacao, 51, 285, 816.

MACARANGA Thou.

M. bicolor Muell. Arg. Linnaea, 34 : 199. 1865-66.

Mindanao, Prov. Surigao, 390.

Endemic.

M. tanarius (Linn.) Muell. Arg. in DC. Prodr. 15: pt. 2, 997. 1866. (Ricinus tunurius Linn.)

Mindanao, Prov. Surigao, 307.

Southern Asia, Malayan region to Australia.

M. tanarius var. tomentosa Muell. Arg. l. c.

Luzon, Manila, 722 ; Prov. Camarines Sur, Pasacao, 114; Mindanao, Prov. Zamboanga, 625 .

Malayan Archipelago. 
M. sp. indet. A very distinct, strongly hispid species, but the material is too inperfect for aceurate identification.

Mindanao, Pror. Surigao, 816.

\section{ACALYPHA Limn.}

A. stipulacea Klotzsch. Nov. Aet. Acad. Nat. Cur. Suppl. $19: 416.1843$. Mindanao, Pror. Surigao, 312; Luzon, Pror. Camarines Sur, Pasacao, 165. Moluceas.

A. sp. indet. Near A. celebica Blume.

Mindanao, Prov. Surigao, 338.

A. sp. inclet. Near A. minahussit Koorders, from Celebes.

Luzon, Prov. Camarines Sur, Pasacao, 815.

\section{HOMONOIA Lour.}

H. riparia lour. Fl. Cochinch. 637. 1790.

Luzon, Prov. Camarines Sur, Pasacao, 159.

Southern Asia, Malayan region, etc. Common on river bars throughout the Philippines.

ALEURITES. Forst.

A. moluccana Willd. Sp. Pl. 4: 590. 1805.

Mindanao, Prov. Yamboanga, Tetuan, 397.

- Southern Asia, Malayan region, etc.

CODI \#UM A. Juss

C. variegatum Blume, Bijdr. 606.1825.

Mindanao, Prov. Zamboanga, 644. Cultivated.

Malayan region.

\section{GELONIUM Roxb.}

G. sp. indet. Near, but distinet from (Y. Lifarium Roxb.

Juzon, l'rov. Camarines Sur, Pasacao, 599.

\section{EXCECARIA Linn.}

E. agallocha Linn. Syst. Nat. ed. 10. 1288. 1758-59.

Luzon, P'rov. Camarines Sur, Pasacao, 115.

Tropical Asia, Malayan region, etc.

\section{HOMALANTHUS Juss.}

H. fastuosus (Muell. Arg.) F. Vill. Nov. App. 196. 1850. (Curumbium fes: (nosum Muell. Arg.)

Mindanao, Prov. Surigao No. 513; also Prov. of Zamboanga No. 385.

Visayan, Bayante.

Endemic.

A species related to IIomalaulhus gigantens, from Java, but differing especially in its entirely glabrous leaves, inore numerous stamens, and nearly spherical fruits.

\section{EUPHORBIA Linn.}

E. atoto Forst. f. Prodr. 36. 1797. (E. halophila MIiq.)

Luzon, Prov. Camarines Sur, Pasacao, 263.

On seacoast, tropical Asia to Australia, etc. 
PLATE III.

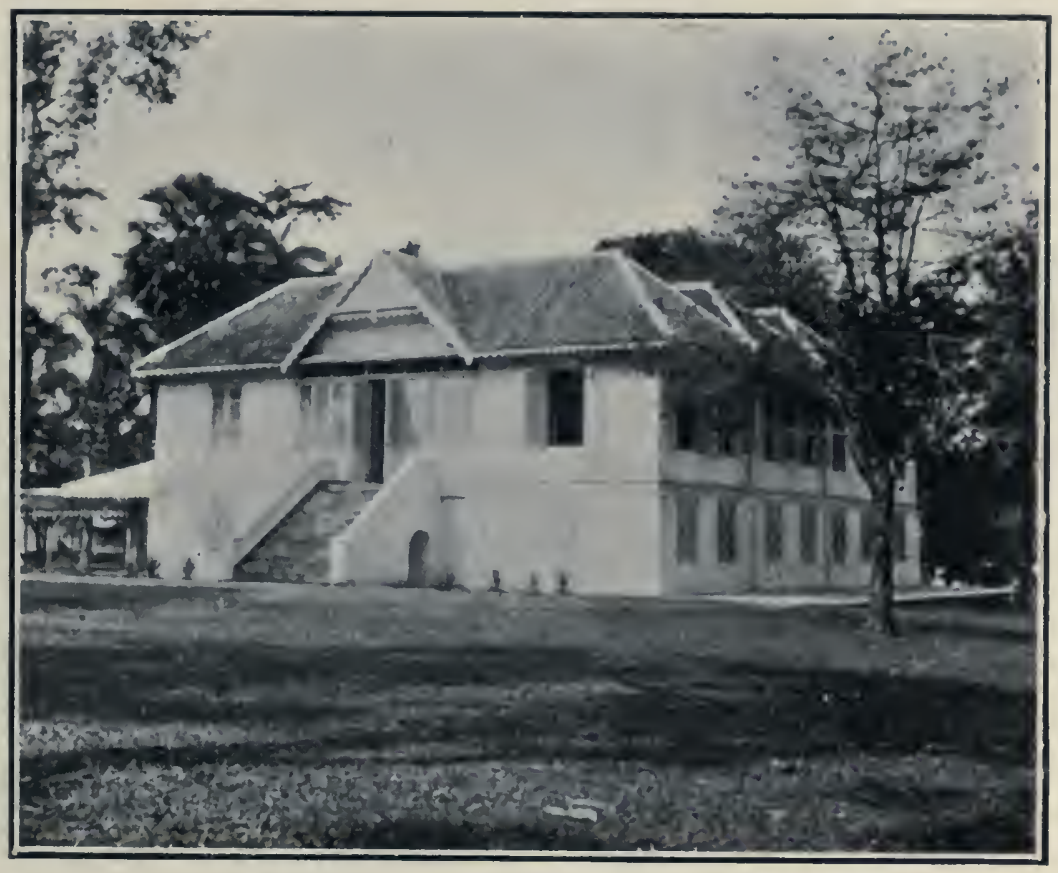

FIG. 1. - HERBARIUM BUILDING, FOREST FLORA INVESTIGATIONS.

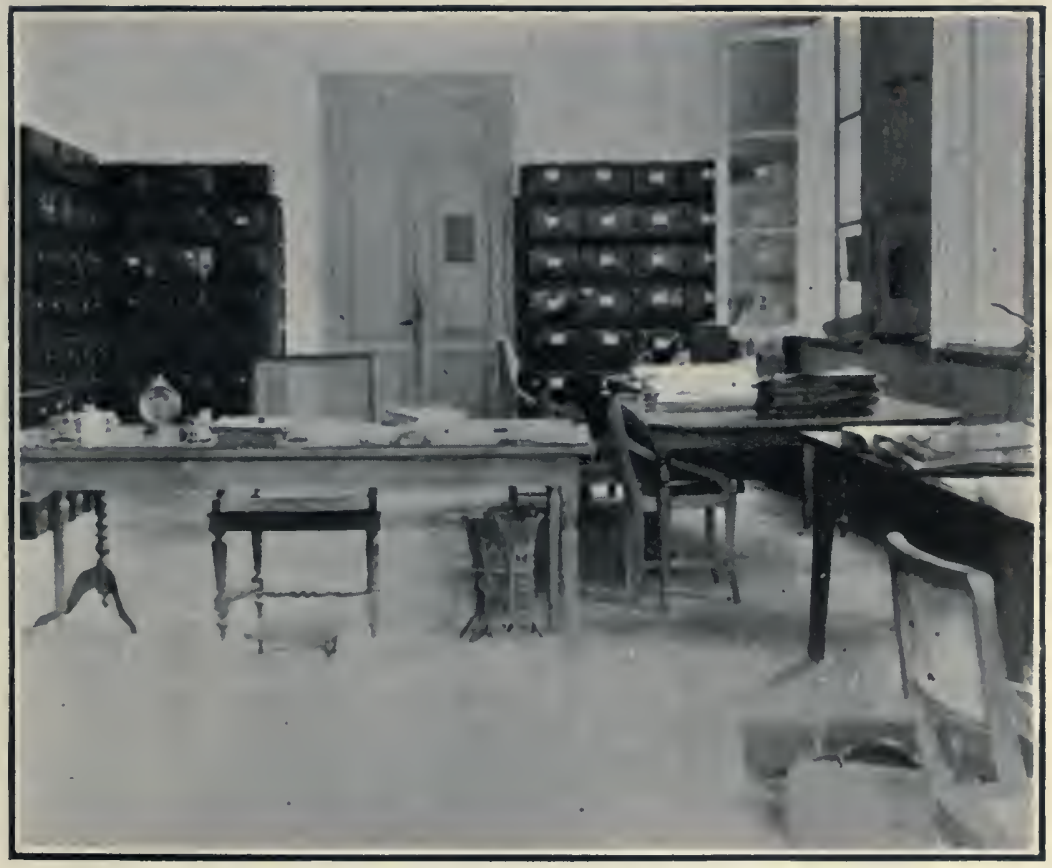

FIG. 2. - LABORATORY FOR SYSTEMATIC BOTANY, FOREST FLORA INVESTIGATIONS. 

PLATE IV

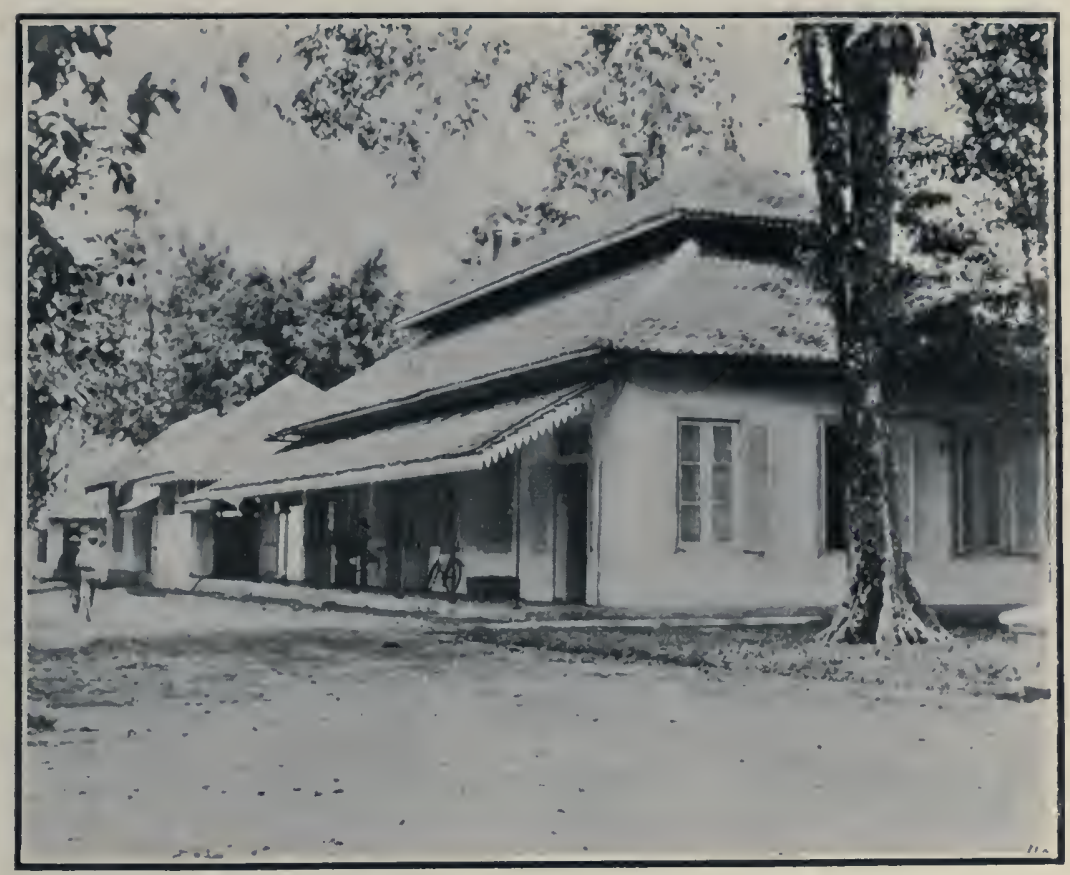

FIG. 1.-OFFICE OF THE EXPERIMENT GARDEN.

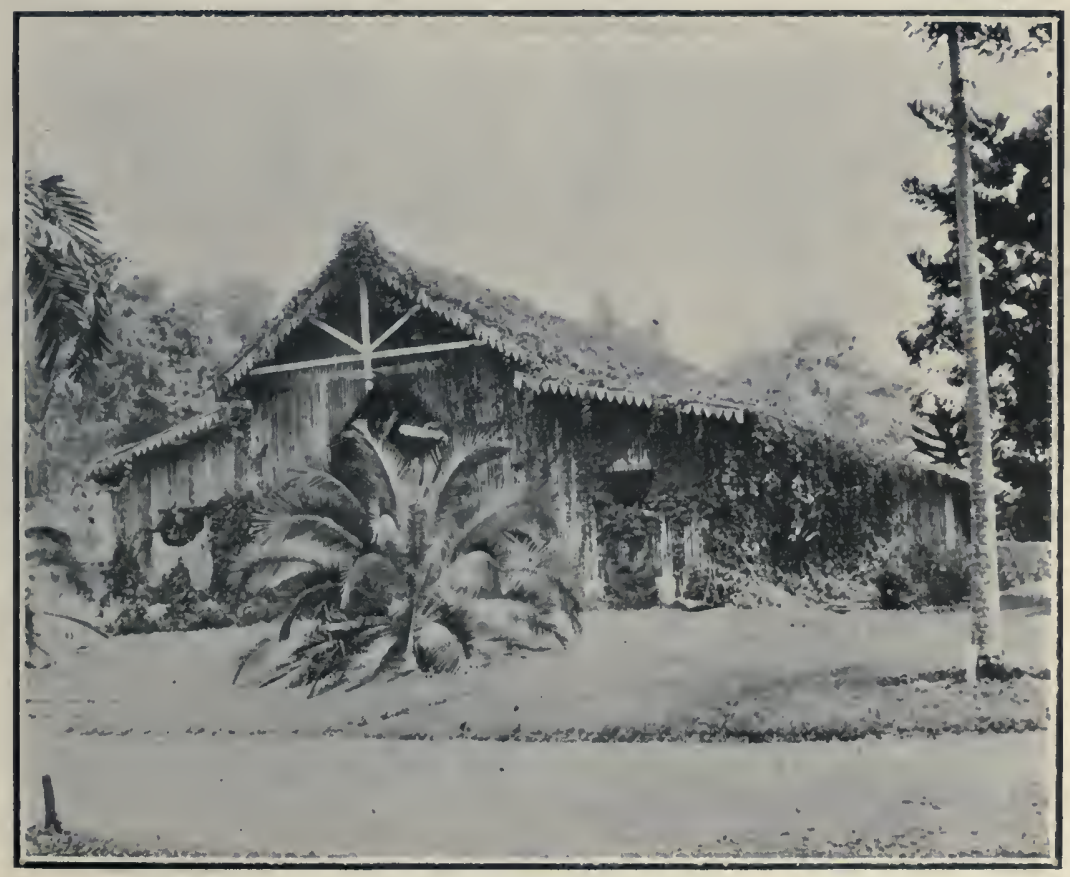

FIG. 2.-AROID HOUSE. 



\section{ANACARDIACEAS.}

\section{BUCHANANIA Spreng.}

B. florida Schauer, Nov. Aet. Nat. Cur. Suppl. 19: 481. 1843.

Luzon, Prov. Camarines Sur, Pasacao, 36; Mindanao, Prov. Surigao, 426, 507.

Taualog, Ligas.

B. florida Schaner, var. arborescens, Engler, in DC. Monog. Phanerog. 4: 189. 1883.

Mindanao, Prov. Zamboanga, 610; Island of Dinagat, 442.

B. florida Schaner, var. petiolaris Engler, l. c.

Iuzon, Prov. Bataan, Mariveles, 764; Prov. Camarines Sur, Pasacao, 827.

$A$ variable species, extending from tropical Asia to the Malayan region.

\section{MANGIFERA Linn.}

M. odorata Griff. Notul. 4: 417. 1849.

Mindanao, Prov. Zamboanga, 658.

Malayan region.

M. cæsia Jack. in Roxb. Fl. Ind. ed. Carey 2: 441.

Mindanao, Prov. Surigao, 345; Prov. Zamboanga, 660. Long-petioled forms.

Malayan region.

M. sp. indet.

Luzon, 89; Mindanao, 651. No flowers or fruit.

\section{ANACARDIUM Linn.}

A. occidentale Linn. Sp. Pl. 383. 1753.

I uzon, Prov. Bataan, Mariveles, 784.

Introduced from tropical America and now extensively cultivated and spontaneous in the Archipelago; known by the natives as cusoy or cachui.

\section{KOORDERSIODENDRON Engl.}

KOORDERSIODENDRON PINNATUM (Blanco) Helicteres pinnata Blaneo,

Fl. Filip. ed. 1, 384. 1837; Cyrtocarpa quinquestila Blaneo, 1. c., ed. 2; 269. 1845; Odinu speciosa Blume, Mus. Bot. Lugd. Bat. 1: 206. 1849-51; Koordersiodendron celebicum Engl. in Mededeel. 's Lands Plant. 19: 410. 1898; Boerlage in Icon. Bogor. 4: 55. pl. 94, 95. 1901.

Luzon, Prov. Camarines Sur, Pasaeao, 121.

Tagalog, Amuguis.

A monotypic genus, extending from the Philippines to Celebes and New Guinea.

\section{SEMECARPUS Linn. f.}

S. perrottetii March, Rev. Anarc. 169. 1869.

Luzon, Prov. Bataan, Mariveles, 769; Manila, 716. Mindanao, Prov. Zamboanga, $510,537,587,600$.

Celebes.

Semecarpus gigantifolia F. Vill. Nov. App. 350. 1883; Vidal, Sinopsis, Atlas, t. 36, A. f. A. 1883 .

Mindanao, Prov. Surigao, 511.

Endemic.

S. MACROPHYLLA Merrill, sp. nov.

A tree, with very large obovate leaves, ample terminal panicles, striate branches and stout, flattened petioles $1.5 \mathrm{~cm}$. long, 8 to $10 \mathrm{~mm}$. broad. Leaves obovate, obtuse, tapering to the cuneate base and rounded apex, $6425-3$ 
4 to $6 \mathrm{dm}$. long, $2 \mathrm{dm}$. wide, firm, coriacious, glabrous and shining above, densely short-pubescent throughont beneath, but especialy on the nerves, midnerve prominent, secondary nerves 18 to 22 , prominent, subparallel and somewhat arcuate, extending to the margins, tertiary nerves freely anasto. mosing. Panicles densely brown-tomentose, beeoming glabrous, $5 \mathrm{dm}$. long, the primary loranches spreading the lower ones about 1.5 to $2 \mathrm{dm}$. long. Fruit, broadly obovate, truncate, and in drying sulcate at the apex, 2.5 to $3.5 \mathrm{~cm}$. long, 2 to $2.5 \mathrm{~cm}$. thick, coverel with a dense irown tomentum. Calyx lohes persistent, lanceolate, acute, 5 to $6 \mathrm{~mm}$. long, densely tomentose. Flowers unknown.

Type specimen No. 348, Surigao, Mindanao, also No. 526 from the same locality belongs here.

A most distinct species, recognized by its large leaves, which are pubescent beneath, its brown-tomentose panicles, fruit, ete.

S. sp. indet.

Luzon Prov. Bataan, Mariveles, 746.

\section{CELASTRACEL.}

\section{LOPHOPETALUM Wight.}

L. sp. indet. A species closely related to $L$. toxicum Loher, Icones Bogoriensis $\mathbf{1}$ : 1. 16. 1897, from central Luzon, but differing somewhat in the inflorescence and floral characters. It is perhaps even more elosely related to $L$. crlebica Loorders, Mededeel, 's Lands Plant. 19:623. 1898, differing somewhat from that species in the inflorescence and leaves.

Mindanao, Prov. Zamboanga, 565.

GYMNOSPORIA Benth. and Hook. f.

G. montana Benth. Fl. Austral. 1: 400. 1863.

Luzon, Prov. Camarines Sur, Pasacao, 98.

Widely distributed in the Tropics.

\section{ICACINACEA:}

\section{GONOCARYUM Miq.}

G. sp. indet. Seems very near $G$. teysmmnicmm Scheff., from the Malayan Archipclago.

Luzon, Prov. Camarines Sur, Pasacao, 86.

\section{S'TAPHYLEACEA:}

T. ep. nov.?

\section{TURPINIA Vent.}

Mindanao, Prov. Surigao, 354.

A species related to, but quite distinct from, both T. sphacrocarpa I3lume, and T. pomifera DC. Specimen in fruit.

\section{SAPINDACEAS.}

\section{ERIOGLOSSUM Blume.}

E. edule Blume, Bijdr. 229. 1825.

Luzon, Prov. Camarines Sur, Pasacao, 135, 215. Mindanao, Prov. Zamboanga, 614 .

Tropical Asia and the Malayan region. 


\section{OTOPHORA Blime.}

O. PINNATA (Blanco) Cupura primntu Blanco, Fl. Filip. ed 1, 644. 1837; (:pupnrufu Blanco, Fl. Filip, ed. 2, 184. 1845, non Iinn.; Otolepis niyrescens Turez. Bull Soc. Nat. Mosc. 21: 572. 1848; Cupura niyrescens Vidal, Rev. Pl. Vasc. Filip. 97. i886.

Luzon, Prov. Bataan, Mariveles, 750.

Findenic.

TRISTIRA Radlk.

T. triptera (Blanco) Radlk. Act. Congr. Bot. Amst. (1877) 133. 1879. (Melicocen triptera Blanco.)

Luzon, Prov. Camarines Sur, Pasacao, 200.

Endemic.

NEPHELIUM finn.

N. longana (Laun.) Cambess, Mem. Mus. Paris, 18: 30. 1829. (Euphoria longana Iain.)

Luzon, Prov. Bataan, Mariveles, 756; Prov. Camarines Sur, Pasacao, 79.

Tagalog, Alupag or Ilalupac.

Tropical Asia.

N. sp. indet.

Luzon, Prov. Camarines Sur, Pasacao, 204.

\section{ELLATOSTACHYS Radlk.}

E. verrucosa (Blume) Radlk. Sitzb. Acad. Muench. 9: 601. 1879. (C'upania verrucosa Blume, Rumphia, $3: 161$. 1825.)

Mindanao, Prov. Zamboanga, 530, 604.

Java.

- L. sp. indet

\section{LEPIDOPETALUM Blume.}

Mindanao, Prov. Zamboanga, 546, 562.

Specimens in fruit, very closely related to $L$. subelichotomum Radlk., from German New Guinea, Hollrung No. 337 in Herb. Hort. Bog. Bogor.

\section{HARPULLIA Roxb.}

H. blancoi F. Vill. Nov. App. 53. 1883.

Luzon, Prov. Camarines Sur, Pasacao, 43.

In Index Kewensis, IIarpulia blancoi and its numerous synonyms is reduced to II. cupanoides Roxb., but here following Vidal, is held distinct.

Endemic.

\section{VI'TACEA.}

\section{VITIS Linn.}

V. adnata Wall. Cat. No. 5998. 1828.

Luzon, Prov. Camarines Sur, Pasacao, 230.

Tagalog, Bulacan.

Tropical Asia, Malayan region to Australia.

V. sp. indet. Seems near $V$. javanica Spreng, but the material is very poor and imperfect.

Luzon, No. 294. 


\section{LEEA Jinn.}

L. javanica Blune, Bijdr. 197. 1825.

Iuzon, Prov. Camarines Sur, Pasacao, 190, 805. Mindanao, Prov. Surigao, 332.

Malayan region.

L. sambucina Willd. Sp. Pl. 1:1177. 1797.

Mindanao, Prov. Surigao, 310.

Appears to be a large-leafed form of this species, which is found throughout the Tropics of the Old World.

L. sp. indet.

Mindanao, Prov. Surigao, 668; Prov. '/amboanga, 582. Specimens very imjerfect.

\section{ELAEOCARPACEA.}

\section{ELFOCARPUS Iinn.}

* E. floribundus Blume, Bijdr. 120. 1825.

Mindanao, Prov. Surigao, 403.

Tropical Asia.

E. sp. indet. Near E. floribundus Blume, but distinct.

Surigao, 382. No. 352 from Surigao represents another species, immature, and therefore not possible to identify readily.

\section{MUNTINGIA Linn.}

M. calabura Linn. Sp. Pl. 509. 1753.

Manila, 719 .

A species introduced from Mexico, and now common about towns.

\section{TILIACE.T.}

\section{DIPLODISCUS Turez.}

D. paniculatus Turcz. Bull. Soc. Nat. Mose. 31: pt. 1, 235. 1858.

Luzon, Prov. Camarines Sur, Pasacao, 10. Mindanao, Prov. Surigao, 376.

A inonotypic endemic genus.

\section{GREWIA Jinn.}

G. lævigata Vahl. Symb. Bot. 1:34. 1790.

Iuzon, Prov: Camarines Sur, Pasacao, 214.

Tropical Asia and Africa, Malayan region.

G. sp. indet.

Manila, No. 734. A form related to $G$. Liviguta, but with unuch smaller leaves than that species; Pasacao, No. 55, probably an undescriber species.

COLONA Cav.

C. serratifolia Cav. Ic. 4: 47 1. 370. 1797. (Columbia americuma Pers. Syı. Pl. 2: 66. 1807; C. serratifolia DC. Prodr. 1: 512. 1824; C. imequalerata Turez. Bull. Soc. Nat. Mosc. 31: 233. 1858; ( (anilao Blanco, Fl. Filip. ed. 1, 65t. 1837.)

Juzon, Prov. Camarines Sur, Pasacao, 90, 792. Mindanao, Prov. Zamboanga, 639; Prov. Surigao, 320; Jrov. Davau, 673.

Tagalog, Anilno.

A variable endemic species. C. javanicu is perhaps not specifically distinct from this species.

C. sp. nov.?

Luzon, Prov. Bataan, Mariveles, 749. 


\section{MALVACEA.}

\section{HIBISCUS Iinn.}

H. tiliaceus Linn. Sp. Pl. 694. 1753.

Lnzon, Manila, 739, 793; Prov. Camarines Sur, Pasacao, 94, 333. Mindanao, Prov. Surigao, 824.

Tagalog, Bancilo.

Widely distributed in the Tropics.

\section{THESPESIA Soland.}

F. macrophylla Blume, Bijslr. 73. 1825.

Luzon, Prov. Camarines Sur, Pasacao, 147, 209.

Tropical Asia, Africa, the Malayan region, etc.

\section{GOSSYPIUM Linn.}

G. herbaceum Linn. Sp. Pl. 693. 1753.

Mindanao, Prov. Surigao, 510 bis; Bongao Island, 843.

Tagalog Gupus, Cuebus.

Cultivated.

\section{BOMBACAEAE.}

CEIBA Medik.

Ceiba pentandra Gaertn. Fruct. 2: 244. t. 133. 1791. (Eriodendron anfractuosum DC.)

Luzon, Prov. Camarines Sur, Pasacao, 4.

Tropical Africa and Asia, the Malayan region, etc.

\section{S'TERCULIACEA.}

\section{MELOCHIA Linn.}

M. corchorifolia Linn. Sp. Pl. 675. 1753.

Mindanao, Prov. Surigao, 688.

Widely distributed in the Tropies of both hemispheres.

M. arborea Blanco, Fl. Filip. ed. 1, 524. 1837. (M. velutina Bedd.)

Mindanao, Prov. Surigao, 340; Prov. Zamboanga, 630.

Visayan, Banitlon.

Endemic.

COMMERSONIA Forst

C. platyphylla Andr. Bot. Rep. tt. 603, 519. 1799-1811.

Mindanao, Prov. Surigao, 684.

Malayan region.

\section{PTEROSPERMUM Schreb.}

P. diversifolium Blume, Bijdr. 88. 1825.

Luzon, Prov. Camarines Sur, Pasacao, 15.

Tagalog, Bayoc or Bayog.

Southern Asia and the Malayan region.

P. blumeanum Korth, in Nederl. Kruidk. Arch. 1: 311. 1848.

Luzon, Prov. Camarines Sur, Pasacao, 66. Mindanao, Prov. Zamboanga, 542, 640.

Visayan, Bayuc-bayucren or Buyog-bayog.

Sumatra and Java. 
KLEINHOVIA Linn.

K. hospita Linn, Sp. Pl. el. 2, 1365. 1763.

Iuzon, Prov. Bataan, Balanga, 847; Prov. Camarines Sur, Pasacao, 822. Minda.nao, I'rov. Surigao, 328; Prov. Zamboanga, 636.

Visayan, Tanag.

Tropical Asia and the Malayan region.

STERCULIA Jinn.

S. Laevis Wall. Cat. No. 1138. 1828.

Luzon, Manila, 713.

Malayan Peninsula, Java.

S. stipularis R. Br. in Benn. Pl. Jav. Rar. 232. 1844.

Juzon, Prov. Camarines Sur, Pasacao, 249.

Findenic.

S. sp. indet.

Inzon, Manila, 710, 724.

A species apparently near S: peltutr Blume, but differing in some essential characters.

S. sp. inclet.

Mindanao, J'rov. Camarines Sur, Pasacao, 207.

A form near and probably identieal with S. hulmulurire Scheff., from the Island of Halmahaira.

S. sp. indet.

Luzon, Prov. Camarines Sur, Pasacao, 60.

TARRIETIA Blume.

T. SYLVATICA (Vidal). (Heritiera sylutica Vidal, Rev. Pl. Vase. Filip. 66. 1886; Sterculia nolrilis F. Vill. non R. Br.)

Luzon, Prov. Camarines Sur, Pasacao, 88.

Tagalog, Dungor.

Endemic.

The proper genus of this plant was indicated to me by Dr. S. H. Koorders, and while in Java I drew up a description of the species with the intention of cledicating it to 1)r. Koorders. On my return to Manila, however, I had occasion to look up the description of Heritiera sylumtien Vidal, while identifying another specimen, and consider that No. 88 of this collection is identieal with Vidal's species, and that the proper genus is Iurretiu rather than Heritiru. I have not seen the type material of Vidal's species, which will be found in the Kew herbarium. This species is perhaps most closely related to T. s!mmolicifolin Mast., from the Malayan Peninsula, at least in the vegetative characters of its simple leaves, but from which it is distinguished by its much smaller, narrower, acute or aruminate leaves which are silvery white beneath, smaller fruits, etc. From all the other described species in the genus it differs in its unifoliate leaves.

\section{PETEROCYMBIUM R. Br.}

P. javanicum R. 13r. in Benn. Pl. Jav. Rar. 219. t. 45. 1844. (Sterculia cumpumeluta Wall.)

luzon, Prov. Camarines Sur, Pasacao, 124.

Tagalog Tiloto.

Burma, Malayan Peninsula and Archipelago. 
HERITIERA Ait.

H. littoralis Dryand, in Ait. Hort. Kew. 3:546. 17 S9.

Luzon, Prov. Caunarines Sur, Pasacao, 65. Mindanao, Prov. Surigao. 413.

Tagalog, Inugom lute.

Widely distributed in the Tropics.

\section{DILLENIACEAS.}

\section{DILLENIA Linn.}

D. philippinensis Rolfe, Journ. Linn. Soc. Bot. 21: 307. 1884.

Luzon, Prov. Camarines Sur, Pasacao, 54; Prov. Bataan, Mariveles, 781.

Tagalog, Cutmon.

Endemic.

D. philippinensis Rolfe, Var.?

Mindanao, Prov. Surigao, 308; Prov. Zamboanga, 836.

$A$ form with the under sides of the leaves, young branches, and sepals pubescent or hirsute. Perhaps a distinct species.

\section{SAURAUIA Willd.}

* S. reinwardtiana Blume, Bijdr. 128. 1825.

Mindanao, Prov. Surigao, 337.

Java.

\section{THEACEN.}

\section{TERNSTRCEMIA Nutis.}

T. toquian F. Vill. Nov. App. 19. 1883.

Luzon, Prov. Canarines Sur, Pasacao, 57.

This species seems very near to T. aneura Miq.

Celebes.

\section{GU'T'IIIEREAE. \\ CRATOXYLON Blume.}

*C. celebicum Blume, Mus. Bot. Lugd. Bat. 2 : 16.1856.

Luzon, Manila, 728. Mindanao, Prov. Surigao, 358.

Celebes.

C. floribundum (Turez.) F. Vill. Nov. App. 16. 1883 (Ancistrololnts floribundus Turez.)

Luzon, Tarlac, 737; Prov. Camarines Sur, Pasacao, 828.

Endemic.

C. sp. indet.

Luzon, 31; Mindanao, 655.

CALOPHYLLUM Linn.

C. inophyllum Linn. Sp. PI. 513. 1753.

Luzon, Prov. Camarines Sur, Pasacao, 22, 735.

Tagalog, Pelumario.

Widely distributed in the Tropics. 
C. wallichianum Planch. and Triana, Mém. Gutt. $272 . \quad 1862$.

Pasacao, Prov. Camarines Sur, Luzon. 53, 144, Feb.-March, 1902; also collected at Mariveles, Prov. Bataan, Luzon, 579, Jan. 21, 1902.

Tagalog, Palo-muric or P'ulo marin de monte.

A very distinct species, at once recognized by the peculiar brown-tomentose branches, inflorescence, and sepals.

C. sp. indet.

Luzon, Prov. Camarines Sur, Pasacao, 240.

\section{GARCINIA linn.}

G. cumingli Pierre, Fl. Forest. Cochinch. Enum. 11:t. $7 \delta, f . F . E$.

Luzon, Prov. Camarines Sur, Pasacao, 231.

Endernic.

G. duodecandra Pierre, 1. c. $28: 1.84$. f. l:

Luzon, Prov. Camarines Sur, Pasacao, 195.

Tagalog, Gatisan.

Endemic.

G. sp. indet.

Luzon, 78. 259.

\section{IIP'TEROCARPACEA.}

SHOREA Roxb.

* S. scrobiculata Burck, Ann. Jard. Bot. Buitenz. 6:207. 1887.

Mindanao, 840.

Identical with the type material of Burck's species, 2538, 2917 Reccari, from Borneo, in Herb. Hort. Bot. Bog.

S. spp. indet.

Luzon, 104, 129; Mindanao, 356.

D. sp. indet.

DIPTEROCARPUS Gaertn. f.

Luzon, Prov. Bataan, Mariveles, 755.

Tagalog, Punuo.

\section{BIXACEA:}

BIXA Linn.

B. orellana Linn. Sp. Pl. 512.1753.

Luzon, Manila 740.

Introduced from America.

Tagalng, Achiote.

\section{FIACOUR'TLAEA}

\section{HYDNOCARPUS Gaertn.}

*H. heterophylla Blume, Rumphia, 4: 22. t. 178 B. f. l. 1848. (Turaktogenos blumei Hassk.)

Luzon, Prov. Camarines Sur, Pasacao, 111, 287.

Visayan, Butong memor.

Java and Sumatra.

R. sp. indet.

RYPAROSA Blume.

Mindanao, Prov. Surigao, 416 .

Material very imperfect. 
SCOLOPIA schreh.

S. sp. indet. Very near s. roxburghii Clos.

Island of Dinagat, 482.

HOMALIUM Jacq.

H. villarianum Vidal, Rev. Pl. Vasc. Filip. 142. 1886.

Luzon, Prov. Camarines Sur, P'asacao, 24, 72. Mindanao, l'rov. Surigao, 469.

Tagalog, Aranga.

Endemic.

FLACOURTIA Juss.

F. cataphracta Roxb. in Willd. Sp. Pl. $4: 830.1804$.

Mindanao, Prov. Surigao, 471 .

Tropical Asia and the Malayan region.

F. sp. indet. Near F. rumontchi L'Herit.

Luzon, Tarlac, 703.

CASEARIA Jacq.

C. cinerea Turcz. Bull. Soc. Nat. Mosc. 31: 462.1858.

Luzon, Manila, 729 .

Endemic.

${ }^{*}$ C. moluccana Blume, Mus. Bot. Lugd. Bat. 1: 255. 1849-1851.

Mindanao, Prov. Surigao, 414; Island of Dinagat, 467.

Amboina.

C. sp. indet.

Luzon, 64, 276.

\section{DATISACEA.}

\section{OCTOMELES Miq.}

O. sumatrana Miq. Fl. Ind. Bat. Suppl. 336. 1860.

Luzon, Prov. Camarines Sur, Pasacao, 16.

According to Warburg, the Philippine material represents a distinct species, I). moluccana Warb., which extends from the Philippines to Amboina, Celebes and New Guinea, Q. sumutrana Miq., being confined to the western portion of the Malayan Archipelago.

\section{THYMELAACEA.}

\section{GYRINOPSIS Decne.}

G. cumingiana Decne. Ann. Soc. Nat. II. 19: 41. t. 1. 1843.

Luzon, Prov. Camarines Sur, Pasacao, 236.

A monotypic endemic genus, previously known only from the original collection, Misamis, Mindanao; 1617 Cuming.

\section{PHALERIA Jack.}

P. cumingii (Meissn.) F. Vill. Nov. App. 183. 1883. (Drimyspermum cumingii Meissn.)

Luzon, Prov. Camarines Sur, Pasacao, 150, 282, 283. Mindanao, Prov. '/amboanga, 575 .

Fndemic. 


\section{LYTHRACER. \\ PEMPHIS Forst.}

P. acidula Forst. Char. Gen. 68. t. \&4. 1776.

Luzon, Prov. Camarines Sur, Pasacao, 168, 178, 251.

A widely distributed species, found on the seacoasts from East $\mathrm{A}$ frica, Southern Asia, to Australia, etc.

\section{LAGERSTROEMIA Linn.}

L. speciosa (Linn.) Pers. Syn 2: 72. 1807. (Munchausia speciosa Linn; L. flosreginic Retz.)

Luzon, Tarlae, 701. Mindanao, Prov. Surigao, 302.

Tagalog, Banabu.

Southern Asia to Australia.

L. batitinan Vidal, Rev. Pl. Vase. Filip. 139. 1886. (L. heptuptert Vidal, Sinopsis, t. 52. f. A., non Mliq.)

Luzon, Prov. Camarines Sur, Pasacao, 137. Mindanao, Prov. Davao, MIt. Apo. 680.

Endemic.

\section{LAWSONIA Linn.}

L. inermis Linn. Sp. Pl. 349. 1753. ( $L_{\text {s }}$ alba Lam. )

Mindano, Prov. Zamboanga, 611, 634.

Widely distributed in the tropical region of the Old World, introduced in the

Philippines, and commonly eultivated for ornament, also growing wild abont towns etc.

\section{SONNERTIACEAS. \\ SONNERATIA Linn. f.}

S. pagatpat Blaneo, Fl. Filip. ed. 1, 424. 1837.

Luzon, Prov. Camarines Sur, Pasacao, 68.

This species is evidently distinet from $S$. acidu Linn. f., to which it was referred by

Fernandez-Villar. It may be the same as $S$. obovatu Blume.

\section{DUABANGA Hanilt.}

D. moluccana Blume, Mus. Bot. Luggd. Bat. 1: 109. 1849-51.

Luzon, P'rov. Camarines Sur, Pasacao, 106, 158, and (?) 210; Mindanao, Prov. '/amboanga, 541, 615.

Tagalog, Julitul.

Java and Borneo.

\section{LEYTHIDACEAS.}

BARRINGTONIA Forst.

B. speciosa Forst. Char. Gen. 76. 1. $3 S . \quad 1776$; Linn f. Suppl. 312.

Luzon, Prov. Camarines Sur, Pasacao, 3.

Malayan Archipelago, Islands of the Pacific, ete.

B. racemosa Blume, in DC. P'rodr. 3: 288. 1828.

Luzon, Manila, 708; Prov. Bataan, Mariveles, 775; Mindanao, Prov. Surigao, 319 , sinall form.

Tagalog, Juted or Putrt.

Southern Asia, Malayan Archipelago, and Polynesia. 


\section{RHIZOPHORACEA:}

\section{GYNOTROCHES Blume.}

*G. axillaris Blume, Bijdr. 219. 1825.

Minclanao, Prov. Surigao, 485; Island of Dinagat, 491.

On the seacoasts of the Malayan Archipelago.

\section{CERIOPS Arns.}

C. candolleana Arn. Ann. Nat. Hist. 1: 364. 1838.

Luzon, Prov. Camarines Sur, Pasacao, 71. Mindanao, Prov. Surigao 486, 501.

Seacoasts of Tropical Africa and Asia to Australia.

\section{RHIZOPHORA Linn.}

R. mucronata Lam. Encycl. 6: 169. 1804.

Luzon, Prov. Camarines Sur, Pasacao, 81, 140.

Seacoasts of tropical Africa and Asia to Australia.

\section{BRUGUIERA Linn.}

B. gymnorrhiza Lam. Illus. $t$. $39 \%$. 1791.

Mindanao, Prov. Surigao, 497; Island of Tinago, 430.

Coasts of tropical Africa, Southern Asia, to Australia, Polynesia, etc.

B. eriopetola Wight and Arn. Ann. Nat. Hist. 1: 368. 1838.

Luzon, Prov. Camarines Sur, Pasacao, 70.

Coasts of tropical Asia, Malayan region, etc.

B. parviflora Wight and Arn. Prodr. 311. 1834.

Mindanao, Prov. Surigao, 484.

Distribution as the proceding species.

\section{MYRTACEA:.}

\section{PSIDIUM Linn.}

P. guayava Linn. Sp. Pl. 470. 1753.

Luzon, Prov. Bataan, Mariveles, 778.

Introduced from America, and now common and widely distributed in the Philippines.

\section{DECASPERMUM Forst.}

D. paniculatum (Lindl.) Kurz, Journ. As: Soc. Beng. 46:61. 1877.

Mindanao, Prov. Surigao, 475; Island of Dinagat, 465.

Bengal, through the Malayan Archipelago to Australia.

\section{JAMBOSA DC.}

J. vulgaris DC. Prodr. 3: 286. 1828. (Eugenia jambos Linn. Sp. Pl. 470. 1753.) Luzon, Prov. Bataan, Mariveles, 751.

Widely distributed in the Tropics.

* J. vriesiana Miq. Fl. Ind. Bat. 1: pt. 1, 428. 1855. Mindanao, Prov. Surigao, 834.

Java.

J. Iineata DC. Prodr. 3: 287. 1828. (Eugenia lineutu Duthie.)

Mindanao, Prov. Surigao, 429, 516; Prov. Zaumboanga, 593.

Malayan Peninsula and Archipelago. 


\section{SYZYGIUM Gaertn.}

S. carophyllaceum Gaertn. Fruct. 1: 166. 1. 33. 1788. (Eugeniu carophylleced Wight.)

Mindanao, Prov. Surigao, 424.

Ceylon to Malayan Archipelago.

S. jambolana (Lam.) DC. 1. c. 259. (Ëngenic jonbolena Lam.)

Luzon, Manila, 714; Prov. Bataan, Mariveles, 779; Prov. Camarines Sur, Pasacao, 35.

Tagalog, Lumboi or Dryad.

There are several other numbers in the collection that represent species in this genus, and one or two undeterminable specimens of the genus Jambose. The Philippine species have never been carefully determined in these diflicult genera.

\section{XANTHOSTEMON F, MUell.}

X. verdugonianus Naves in F. Vill. Nov. App. 82. 1883; Fl. Filip .ed. 3, 1. 800 ; Vidal, Sinopsis, Atlas, $1.49 . f . K .1883$.

Island of Tinago, 428 .

Visayan, Muncono.

Endemic.

\section{COMBRETACEA:}

TERMINALIA Linn.

T. catappa Linn. Mant. 2:519. 1767. (T. letifoliu Blanco.)

Luzon, Prov. Camarines Sur, Pasacao, 52, 76.

Southern Asia to Australia and Polynesia.

T. edulis Blanco, Fl. Filip. ed. 2, 265. 1845.

Luzon, Prov. Camarines Sur, Pasacao, 1; Prov. Bataan, Balanga, 306.

Tagalog, Culumpit.

Endenic.

T. calamansanay (Blanco) Rolfe, Journ. Linn. Soc. Bot. 21: 310. 1884. (Gimbernatic calumunsunuy Blanco, FI. Filip. ed. 2, 266. 1845.)

Luzon, Prov. Bataan, Mariveles, 785. Mindanao, Prov. Surigao, 329.

Tagalog, Calumansomay.

Endemic.

T. sp. indet.

Luzon, 785.

QUISQUALIS Linn.

Q. indica Linn. Sp. Pl. ed. 2, 256. 1763.

Luzon, Prov. Camarines Sur, Pasacao, 179, 290.

Widely distributed in the Tropics.

\section{LUMNITZERA Will.}

Lumnitzera purpurea (Gaud.) Presl. Repert. 1: 155. 1834. (Iaguncularia purpurea (iaud. 1826; L. coccinea Wight. and A rn. 1834.)

Island of Dinagat, 441.

Along the seacoasts of Malacca, Malaya Archipelago, to Australia, etc. 


\section{MELASTOMACEA.}

M. spp. indet.

\section{MEMECYLON Linn.}

Four distinet species of this genus are represented in the collection, one or two of them being very eharacteristic and probably undescrilsed. None of the material, however, is in condition for accurate determination, being mostly in fruit, or one number with immature flowers. Nos. 515, 588, 656, 684, 800 .

\section{ASTRONIA Blume.}

A cumingiana Vidal, Phanerog. Cuming. 174. 1885.

Mindanao, Prov. Surigao, 322.

Endemic.

A. macrophylla Blume, Bijdr. 1880. 1825.

Luzon, Irov. Albay, Legaspi, 841.

Borneo, Celebes, ete.

A. rolfei Vidal, 1. c., 174.

Mindanao, Prov. Surigao, 517, 344.

Endemic.

\section{ARALIACEAS.}

S sp. indet.

\section{SCHEFFLERA Forst.}

Luzon, Prov. Camarines Sur, Pasacao, 295. Material very fragmentary.

\section{ARTHROPHYLLUM Blume.}

A. diversifolium Blume, Bijdr. 879. 1825.

Luzon, Prov. Bataan, Mariveles, 753.

Malayan Peninsula and Archipelago.

\section{MYRSINACEAL.}

\section{APSA Forsk.}

M. laxa Mez, in Engler, P'flanzenreich, 9: 32. 1902. (M. imlicu, var. obusu A. DC., and var. wightiana A. DC. in DC. P'rodr. 8: 80. 1844.

Iuzon, Prov. Camarines Sur, Pasacao, 163. Mindanao, 812.

Endemic.

M. hænkeana Mez, l. c. 32 .

Mindanao, Prov. Surigao, 341. Infloreseence longer than deseribed by Mez.

Endemic.

M. spp. indet. 279, 219. The first number from Pasacao is evidently not deseribed by $\mathrm{Me}$ in his reeent monograph of the Hyrsinacer. The last is too imperfeet to identify.

\section{无GICERAS Gaertn.}

A. corniculatum (Linn.) Blanco, Fl. Filip. ed. 1, 79. 1837. (Rhizophoru corniculuta linn. Amoen. Acad. 4: 123. 1760; Umbraculume corniculatum O. Kuntze, Rev. Gen. 2: 405. 1891; Aigiceras majus Gaertn. Fruet. 1: 216. t. 46. 1788.) Mindanao, Prov. Surigao, 431, 498.

Seacoasts southern Asia to Australia.

A. floridum R. and S. Syst. 4: 512.1819.

Luzon, Prov. Camarines Sur, Pasacao, 118, 250, 284.

Seacoasts of Philippines, Amboina, and New Guinea. 


\section{ARDISIA Sw.}

A. philippinensis A. DC. in DC. Prodr. 8: 128. 1844.

Mindanao, Prov. Zamboanga, Tetuan, 534.

Findemic.

A. humilis Vahl. Symb. Bot. 3: 40.1794.

Luzon, Prov. Camarines Sur, Pasacao, 806, 242, and (?) 101; Mindanau, Prov. Surigao, 692, 462; Prov. Zaunboanga, 572.

Southern Asia and the Malayan region.

A. perrottetiana A. DC. Ann. Soc. Nat. II. 14: 95. 1841.

Mindanao, Prov. Surigao, 453, 670, 686.

Endernic.

A. spp. indet. 272,596 . No. 599 is a very distinet form of the subgenus Pyrgus, evidently undescribed by Mez.

RAPANEA Aubl. (Myrsine R. Br.)

R. philippinensis (A. DC.) Mez, in Engler's Pflanzenreich 9: 364. 1902. (Myrsine philippinensis A. DC. in DC. Prodr. 8: 94. 1844.)

Island of Dinagat, 444 .

Endemic.

\section{SAPOTACEAE.}

ILLIPE Kœnig.

I. BETIS (Blaneo) (Azuola betis Blaneo, Fl. Filip. ed. 1, $402 . \quad 1837$; Payena betis F. Vill. Nov. A pp. 125. 1883.)

Luzon, Prov. Camarines Sur, Pasacao, 131, 241.

Tagalog, Betis.

Fndemic.

I. sp. indet.

Mariveles, Prov. Bataan, Lizon, 762.

PALAQUIM Blanco.

P. latifolium Blanco, Fl. Filip. ed. 1, 404. 1837.

Luzon, Manila, 738.

Tagalog, Pelac-pulac.

Endemic.

PALAQUIUM AHERNIANUM Merrill, sp. nov. A tree with obovate, obtuse, or acute leaves which are densely rusty-tomentose beneath, rustytomentose flowers and ellipsoid one-seeded fruits. Young branches with numerous, rather prominent wart-like growths which bear the flowers. Leaves obovate, or sometimes nearly ovate, 12 to $20 \mathrm{~cm}$. long, 6 to $9 \mathrm{~cm}$. wide, the hase acute, the apex obtuse or somewhat acute, glabrous above, densely rustytomentose beneath, the nérves, 15 pairs, prominent, especially beneath; petioles $2.5 \mathrm{~cm}$. long, rusty-tomentose. Flowers numerous, single or fasciculate in twos or threes on wart-like growths on the stem below the leaves, the peduncles 2 em. long, rusty-tomentose. Calyx lobes, $4 \mathrm{~mm}$. long triangular, aeute, rustytomentose. Petals unknown. Ovary rusty-tomentose. Fruit one-seeded, ellipsoid, $2.5 \mathrm{~cm}$. in dianeter.

Type specimen No. 842, Prov, of Zamboanga, Mindanao, also No. 608 Prov. of Zamboanga, Mindanao, June, 1901. 
This species is related to Pulurguium lubbiunum Burck, from Ternate, bnt differs from that species in the different ehape and nervation of ity leaves, longer peduncles, and other characters.

* P. colebicum Burck, Ann. Jard. Bot. Buitenz. 5: 32. 1886. Mindanao, Prov. of Cottabato 839. The specimen is without fruit or llowers, but is undoubtedly this mperies.

Celehes.

P. cuneatum Vidal, Sinopsis, Atlas, 1. 62. f. A. 1883, not Bursiu cumetre Blume. Dichopsis cumeata F. Vill. Nov. $\Lambda_{\mathrm{P} p}$. 124. 1883.

Luzon, Prov. Camarines Sur, Pasacao, 42.

Endemic.

P. sp. indet.

Mindanao, Prov. Cottabato, 837. A species related to $P$. celflirum but distinet. Material very imperfect.

PAYENA $A$. DC.

* P. leerii (Teysul. and Binn.) Benth. and Hook. f. (ien. ['lant. 2: 6i59; Burck, Ann. Jard. Bot. Buitenz. 5: 56. t. \&. 1886.

Islands of Tawitawi, 838 .

Sumatra and Borneo to Amboina.

P. sp. indet.

Island of Dinagat, 483.

MIMUSOPS linn.

M. elengi Linn. Sp. Pl. 349.1753.

Mindanao, Prov. '/amboanga, Tetuan, 567.

Southern Asia, etc.

\section{EBENACEA.}

MABA Forst.

M. buxifolia Pers. Syn. 2: 606. 1807.

Luzon, Prov. Camarines Sur, Pasaean, 5 bis; Island of Dinagat, 448 ; Island of Tinago, 425.

Sp.-Filip. Ebano.

Tropical Asia, Malayan region, ete.

DIOSPYROS Dalech.

D. discolor Willd. Sp. Pl. 4: 1108. 1804.

Luzon, Prov. Bataan, Mariveles, 770; P'ror. Camarines Sur, Paжacao, 29, 169, 278; Mindanao, Prov. Surigao, 360.

Tagalog, Camagom.

D. philippinensis A. DC. in DC. Prodr. 8: 231. 184.

Luzon, Pror. Camarines Sur, Pacacao, 39, 112.

Endemic.

D. pilosanthera Blanco (?), Fl. Filip. ed. 1, 304. 1837.

Luzon, Prov. Camarines Sur, Pasacao, 790.

lindemic.

D. spp. indet.

Luzon 13, 261; Tinago, 427. 


\section{OLEACEAS}

O. sp. indet.

OLEA Linn.

Luzon, Prov. Camarines Sur, Pasacao, 44.

Specimen very imperfect.

MAYAPEA Aubl.

M. sp. indet. Seems near M. rumiflora (Roxb.) F. Muell.

Island of Dinagat, 449,450 .

\section{LOGANIACEAS.}

\section{STRYCHNOS Linn.}

* S. celebica Kioorders, Mededeel 's Lands Plant. 19: 540, 631. 1898. Mindanao, Prov. Zamboanga, Tetuan, 538.

A species previously known only from North Celebes.

\section{COUTHOVIA A. Gray.}

* C. celebica Koorders, 1. c. 537, 538. 1898.

Mindanao, Prov. Surigao, 353.

Visayan, Brali-luali.

A species previously known only from North Celebes.

FAGR正A Thunb.

F. morindæfolia Blume, Rumphia, 2: t. 7s.f. 2, t. 79.1836.

Mindanao, Prov. Surigao, 303; Prov. Zamboanga, 586.

Malayan region.

\section{APOCYNACEAS.}

\section{LEPINIOPSIS Valeton.}

* L. ternatensis Valeton, Ann. Jard. Bot. Buitenz. 12: 252. pl. 28.1895. Mindanao, Prov. Surigao, 693.

That this species should be found in the Philippines is most interesting. This monotypic genus was based on a plant eultivated in the botanical garden at Buitzenborg, supposed to have come from the Island of Ternate, one of the Molnecas. At present, however, the Philippine locality is the only one definitely known for this species in a wild state. The Philippine specimen differs from the type material in Herb. Flort. Bot. Bog., only in its somewhat thicker fruits.

\section{PLUMIERA Linn.}

P. acutifolia Yoir. in Lam. Encycl. Suppl. 2: 667. 1817.

Luzon, Prov. Bataan, Mariveles, 782; Mindanao, I'rov. Surigao, 663.

Tatalog, Carhuchi.

Introduced from America. Cultivated.

ALSTONIA R. Br.

A. macrophylla Wall. Cat. No. 1648. 1828.

Luzon, Prov. Camarines Sur, Pasacao, 75.

Malayan Peninsula. 
PLATE V.
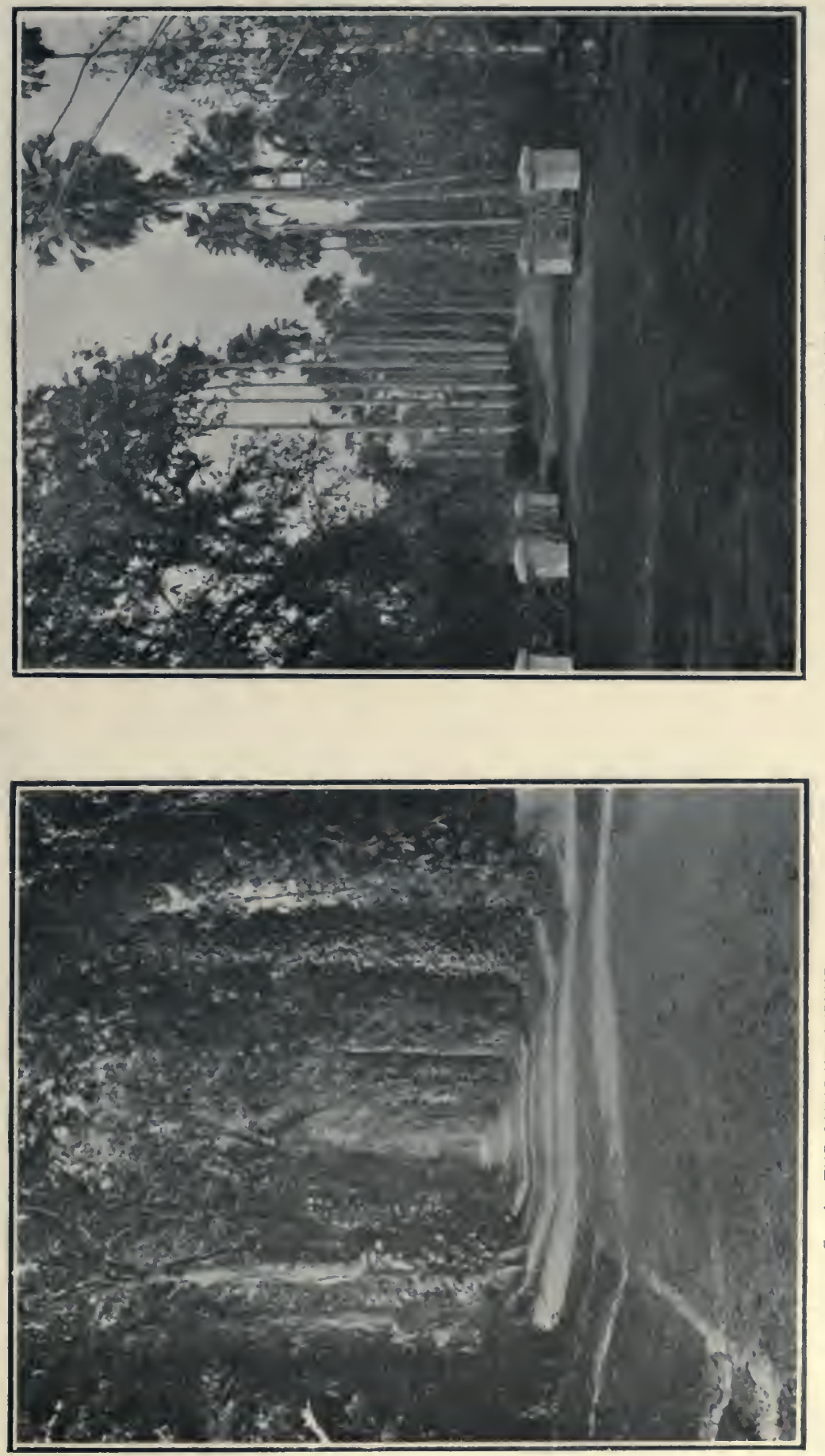

PLATE VI.

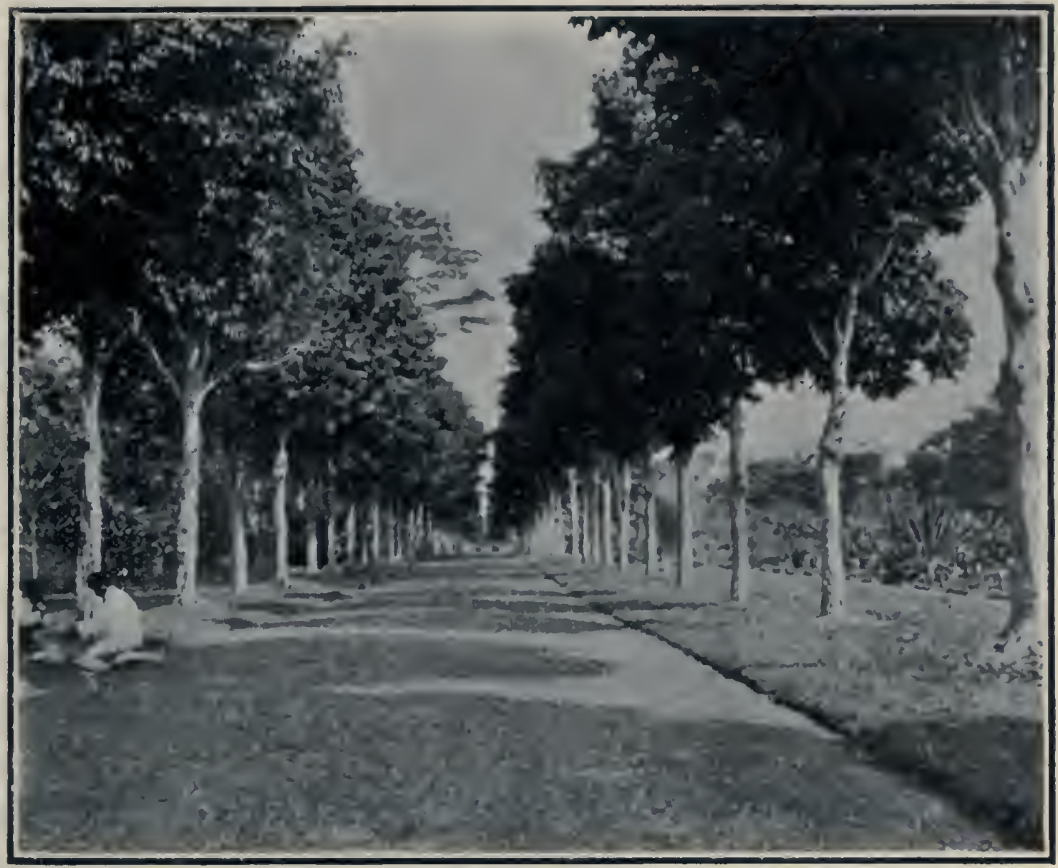

Fig. 1.-THE NEW CANARIUM AVENUE.

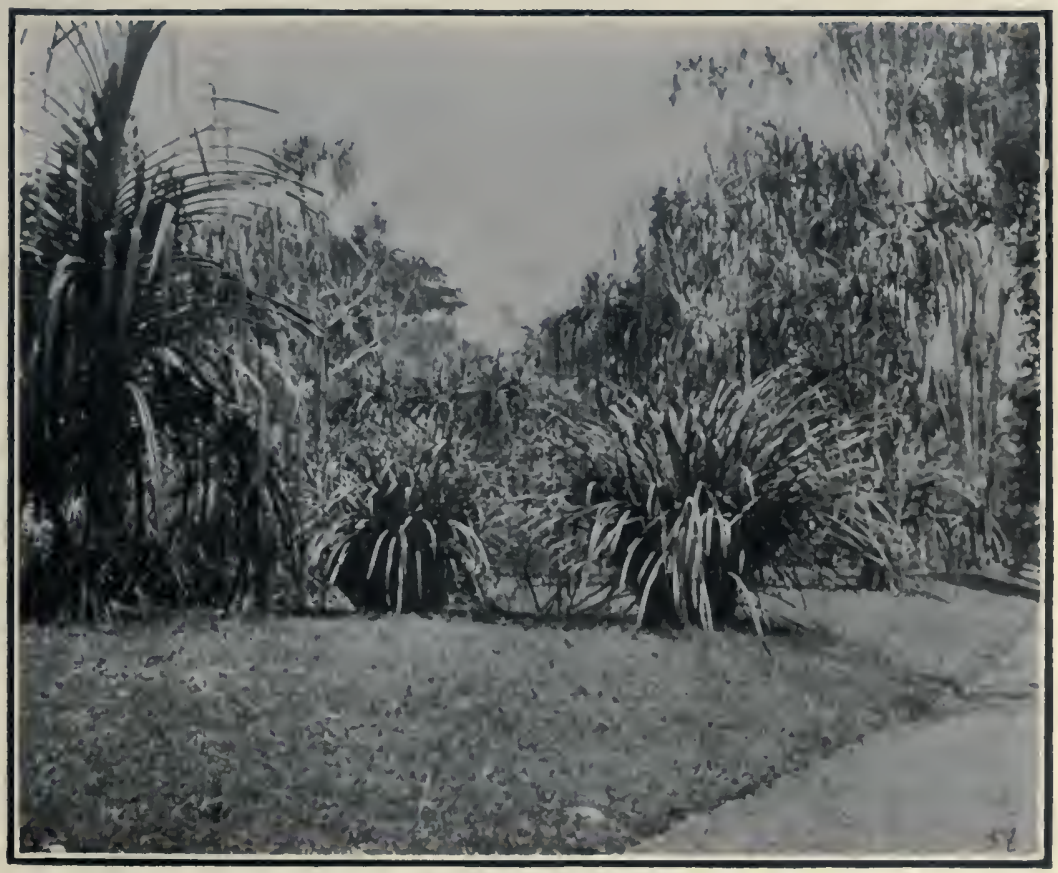

FIG. 2,-A VIEW IN THE PANDANUS SECTION, 

A. scholaris (Linn.) R. Br. Mem. Wern. Soc. 1:75. 1809. (Echites acholaris Linn.) Mindanao, Prov: Zamboanga, 606.

Tagalog, Ditua.

Throughout the Fast Indies.

A. sp. indet.

Luzon, Prov. Camarines Sur, Pasacao, 258.

An imperfect specimen, with inuch smaller leaves than the preceding species.

TABERN 届MONTANA Linn.

T. pandacaqui Poir. in Lam. Encyel. 7: 529. 1806. (T. Laurifolin Blanen, non Linn.)

Luzon, Manila, 717; Prov. Bataan, Mariveles, 772.

Tagalog, Pundacaqui.

Endenic.

T. sphærocarpa Blume, Bijdr. 1028. 1826. (T. globosa Naves.)

I.uzon, Prov. Bataan, Mariveles, 774 .

Tagalog, Punducal.

T. sp. indet.

Mindanao, Prov. Surigao, 311; Island of Dinagat, 489.

Visayan, Jandayagcot.

VOACANGA Dup. Th.

V. cumingiana Rolfe (?) Journ. Linn. Soc. Bot. 21: 313.1883.

Mindanao, Prov. Surigao, 330.

Endemic.

RAUWOLFIA Linn.

R. amsoniaefolia A. DC. in DC. Prodr. 8: 338. 1844.

Luzon, Irov. Camarines Sur, Pasacao, 100, 849.

Endemic.

\section{CERBERA Linn.}

C. odollam Gaertn. Fruct. 2: 193.1791.

Luzon, Prov. Camarines Sur, Pasacao, 34, 799; Island of Dinagat, 438.

Tagalog, Bayac uвa.

Troplical Asia, Malayan region, etc., to Australia.

\section{TRACHELOSPERMUM Linn.}

T. sp. inclet. Near T. frugruns Hook. f., from the Himalaya region.

Luzon, Prov. Camarines Sur, Pasacao, 300. Material in poor condition.

\section{WRIGHTIA R. Br.}

W. sp. nov.? An entirely glabrous form related to $W$. coccinea Sims. Specimen in fruit.

Luzon, Prov. Camarines Sur, Pasacao, 40.

\section{ASCLEPIADACEAS.}

HOYA R. Br.

H. multiflora Blume (?) Bijdr. 1804. 1825.

Mindanao, Prov. Surigao, 495.

Material inperfect, but doubtless this species.

Malayan region.

6425 


\section{CONVOLVUIACEA.}

IPOMGEA Linn.

I. peltata (Limn.) Choisy, لlem. Soc. Phys. Genev. 6: 452. 1833. (Conrolrulus peltatus Linn.)

Luzon, Prov. Camarines Sur, Pasacao, 226, 297.

Hallier places this species in the genus .Merremia.

Malayan region.

I. denticulata (Desrouss.) Choisy, l. c. 447. (Convolvulus denticulatus Desrouss.)

Luzon, Prov. Camarines Sur, Pasacao, 298.

From the Seychelles to Australia and Polynesia.

I. pes-capræ (Linn.) Roth, Nov. Sp. Pl. 109. 1821. (Convolrulus pes-caprir Linn; Ipomoen biloba Forsk.)

Inzon, Prov. Camarines Sur, l’asacao, 299.

Near the seacoast in Tropics of both hemispheres.

\section{BORRAGINACEA.}

CORDIA Linn.

C. blancoi Vidal, Rev. Pl. Vase. Filip. 192. 1886. (C. dichotoma Blanco, non Forst; C. myxa F. Vill., non Linn.; C. sebestena Naves, non allior.)

Luzon, Prov. Camarines Sur, Pasacao, 33, 791.

Endemic.

C. subcordata Lam. Ilustr. No. 1899.

Luzon, Prov. Camarines Sur, Pasacao, 84.

East $A$ frica to Australia and Polynesia.

C. sp. indet.

Luzon, 783.

\section{EHRETIA Jinn.}

E. buxifolia Roxh. P'l. Corom. 1: 42. t. $5 \% .1795$.

Luzon, Prov. Camarines Sur, Pasacao, 810. Mindanao, Prov. Zamboanga, 554,621 .

Southern Asia, Malayan region, etc.

E. philippinensis A. DC. in DC. Prodr. 9: 504. 1845.

Lızon, Prov. Camarines Sur, Pasacao, 152.

Endemic.

\section{TOURNEFORTIA Linn.}

T. argentea Linn. f. Suppl, 133.

Luzon, Prov. Camarines Sur, Pasacao 221, 244.

Southern Asia, Malayan region to Australia, etc.

T. sarmentosa Laun. Illustr. 1: 416. 1791.

Luzon, I'rov. Cainarines Sur, Pasacao, 256.

Philippines to Australia.

\section{VERRENACE.T.}

GEUNSIA Linn.

G. farinosa Blume, Cat. Gew. Buitenz. 48. 1823; Bijdr. 819. 1825.

Mindanao, Prov. Zamboanga, Tetuan, 378.

Malayan region. 
G. cumingiana (Schatuer) Rolfe, Journ. Linu. Soc. Bot. 21:315. 18863. (Cullicarpu cumingimn Schauer.)

Mindanao, Prov. Davao, Mt. A cao, (?) 803.

Eindenic.

\section{CALLICARPA Linn.}

C. angusta Schauer, in 1)C. Prodr. 11: 462. 1847.

Luzon, Prov. Camarines Sur, Pasacao, 198, 811. Minclanao, Prov. Zamboanga, (i62.

Endemic.

C. cana Linn. Mant. 2: 198. 1767.

Luzon, Prov. Camarines Sur, Pasacao, 156.

Southern Asia, Malayan region, to Australia.

* C. arborea Roxb. Hort. Beng. 10. 1814.

Mindanao, Prov. Surigao, 687. No. 540 represent a related form, but quite distinct.

Himalayan region to Sumatra and New Guinea.

\section{TECTONA linn.}

T. grandis Linn. f. Suppl. 151.

Mlindanao, Prov. Zamboanga, 602, 835; Jolo Archipelago, 844.

Southern Asia and Malayan region.

\section{PREMNA Linn.}

P. vestita Sehauer, in DC. P'rodr. 11: 613. 1847 .

Luzon, Prov. Bataan, Mariveles 748, 780; Manila, 718; Tarlac 705.

Tagalog, Alaguo.

Endemic.

P. indet. Seems very near $P$. levigutu Miq., from Sumatra.

Luzon, Prov. Camarines Sur, Pasacao, 138, 801.

P. sp. indet.

Luzon, 103; Mindanao, 355.

\section{GMELINA Linn.}

G. villosa Roxl. Hort. Beng. 46. 1814 .

IInulanao, Prov. Zamboanga, 618; Prov. Surigao, 331.

Southern Asia and the Malayan region.

\section{VITEX Linn.}

V. littoralis Decne, in Nouv. Ann. Mus. Paris. 3: 401. 1834.

Luzon, Prov. Bataan, Mariveles, 752; Prov. Camarines Sur, Pasacao, 37, 826. Mindanao, Prov. Surigao, 389, 504; I'rov. Zamboanga 400, 659. Island of Bongao, $8+5$.

Tagalog, Molave.

Moluceas.

V. negundo Linn. Sp. P'l. 638. 1753.

Luzon, l'rov. Camarines Sur. Pasacao, 166, 255, 223, 814. Mindanao, Prov. Surigao, 671 .

Tagalog, Lagundi.

Southern Asia and Malayan region. 
VITEX PHILIPPINENSIS Merrill, sp. nov.

A tree, nearly glabrous, with ample coriaceous 5 -foliate leaves, and broadly foliatewinged petioles. Branches glabrous, striate. Petioles 12 to $15 \mathrm{~cm}$. long, triangular in cross section, the foliate wings broadly ovate, cordate at the broadly rounded base and also at the apex, the entire breadth $13 \mathrm{~cm}$. I.eaves 5 -foliate, petiolules $0.5 \mathrm{~cm}$. long, leaflets lanceolate-ovate, glabrous, entire, tapering gradually to the cunuate hase, and to the acute or slightly acuminate apex, 30 to $40 \mathrm{~cm}$. long, 10 to $12 \mathrm{~cm}$. wide, lateral veins abont 12 pairs, prominent, anastomosing near the margin. Panicles terminal, glabrous, ample, but shorter than the leaves, $35 \mathrm{~cm}$. long, the longer branches $20 \mathrm{~cm}$. in length, opposite. Bracts lanceolate, acute, $1 \mathrm{~cm}$. long, or less. Flowers numerous, crowded at the ends of the panicle branches, pedicles 2-3 mm. long. Calyx $3 \mathrm{~mm}$. long, 5-toothed, the teeth acute, $1 \mathrm{~mm}$. long. Corolla 6 to $8 \mathrm{~mm}$. long, scurfy-tomentose.

Type specimen collected at Tagana-an, Province of Zamboanga, Mindanao, No. 387 March (?) 1901. Moro (?) Buli-cahoy.

A species readily distinguished by its exceedingly broad foliate petioles, anple 5 -foliate leaves, the former being a most characteristic feature. The aftinity of this species is with Vitex pteropoda Miq. Fl. Ind. Bat. Suppl. 567, from Sumatra, but it is certainly quite distinct from that species.

V. sp. indet.

Luzon, 110, 706.

\section{CLERODENDRON Linn.}

C. intermedium Chain. Linnaea, 7: 150. 1832.

Mindanao, Prov. Surigao, 327.

Endernic.

*C. lanuginosum Blume, Bijdr. 810. 1825.

Mindanao, Prov. Surigao, 691.

A species previously known only from the Moluccas.

* C. minahassæ Teysm, and Binn. Tijd. Ned. Ind. 25: 409. 1863.

Mindanao, Prov. Zamboanga, 568.

A species previously known only from Celebes.

C. sp. indet.

Mindanao, 549. Specimen incomplete.

AVICENNIA Linn.

A. offleinalis Linn. Sp. Pl. 110. 1753.

Luzon, Prov: Camarines Sur, Pasacao, 116, 148.

From East Africa to Polynesia.

\section{BIGNONIACEA:}

\section{DOLICHANDRONE Fenzl.}

D. spathacen (Linn.) K. Schum. Fl. Kaiser Wilhelmsland. 123. 1889. (Bigmonin sputhaceu Linn; D. rheedii Seem.)

Iuzon, Manila 723 (?), Prov. Camarines Sur, P'asacao, 59. Minclanao, Prov. Surigao, 672.

Tagalog, Tui.

Malayan Peninsula and Archipelago, etc.

\section{STEROSPERMUM.}

S. pinnatum (Blanco) F. Vill. Nov. App. 151. 1883.

Luzon, Prov. Camarines Sur, Pasacao, 61. Mindanao, Prov. Surigao, 528, 848. Endemic. 
RUBIACEA.

\section{WENDLANDIA Brtl.}

W. paniculata I)C. Prodr. 4:411. 18:30. (W. luzonensix DC. I. c. 412.)

Minlanao, I'rov. 'Zanboanga, Tetuan, 564.

From the Himalayan region, throughout the Malayan region.

\section{ADINA Salisb.}

A. philippinensis Vidal, Rev. I'l. Vasc. Filip. 148. i886. (A. polycephlulu Vidal, Sinopsis, Atlas, t. 56.f. B., non (Benth.)

Iuzon, P'rov. Camarines Sur, Pasacao, 102.

Endemic.

M. sp. indet.

\section{MITRAGNE Korth.}

Luzon, Prov. Canarines Sur, Pasacao, 21.

NAUCLEA Linn.

N. Blancoi Vidal (?) Phanerog. Cuming. 175. 1855.

Luzon, Prov. Canarines Sur, Pasacao, 58, 808.

Tagalog, Mrembog.

Endemic.

N. spp. indet.

Mindanao, 362, 374, 666.

\section{SARCOCEPHALUS Afzel.}

S. cordatus Miı. Fl. Ind. Bat. 2: 133. 1855-1859.

Mlindanao, Prov. Surigao, 335.

Tropical Asia to Australia.

\section{MUSS无NDA Linn.}

M. grandiflora (Meyen) Rolfe, Journ. Linn. Soc. Bot. 21:311. 1884. (Culycophyllem grundifforum Meyen; M. frondose Blanco, non Linn.)

Luzon, Prov. Camarines Sur, Pasacao, 199, 802, 818. Endenic.

M. anisophylla Virlal, Phanerog. Cuming. 178. 1885. Mindanao, Prov. Surigao, 339.

Findemic.

\section{UROPHYLLUM Wall.}

* U. glabrum Jack. ex Roxb. Fl. Ind. ed Carey, 2: 186.

Mindanao, Prov. Surigao, Placer, 317, 404.

Malayan Peninsula, Java, and Borneo.

\section{RANDIA Houst.}

R. cumingiana Vidal, Phanerog. Cuming. 179. 1885.

Luzon, I'rov. Camarines Sur, Pasacao, 189.

Endemic.

R. sp. indet.

Luzon, 126, Mindanao, 457. 
GARDENIA Ellis.

G. obscura (Blanco) Vidal, Planerog. Cuming. 119. 1885. (Rrmigin olscuren Blanco.)

Luzon, Manila, 720,733 .

Tagalog, Stalubrilues or Culugri.

Endemic.

G. pseudopsidium l3lanco, Fl. Filip. ed 1, 497. $18: 37$.

Luzon, Manila, 744.

Endemic.

TRICALYSIA A. Rich.

T. sp. indet. (Sect. Diplospora.)

Luzon, Prov. Camarines Sur, Pasacao, 248. Seems very near to an unnamed specimen in Herb. Hort. Bot. Bog., collected in Minahassa, North Celebes, by Dr. Koorders. Specimen in fruit.

\section{PLECTRONIA linn.}

P. horrida (Blume) Benth. and Hook. f. ex King in Journ. As. Soc. Beng. 44: 153. 1877. (Canthium horritlum Blume.)

Luzon, Prov. Camarines Sur, Pasacao, 254, 819.

Tagalog, Dayy (q)-d(1y(q).

Malayan Peninsula and Archipelago.

* P. moluccana (Roxb.) Ganthium moluccanum Roxb. Hort, Beng. 85 .

Mindanao, Pros. Surigao. 451.

Moluccas.

\section{GUETTARDA Linn.}

G. speciosa Linn. Sp. l'l. 991.1753.

Luzon, Prov. Camarines Sur, Pasacao, 19, 205; Island of Dinagat, 464.

Extends from East Africa to Polynesia.

\section{TIMONIUS Rumph.}

TIMONIUS PHILIPPINENSIS Merrill, sp. nov.

A shrub (?) or tree with glabrous, terete, purplish ultimate branches, prominent leaf scars and ovate or ovate-oblong, rarely obovate, firm leaves. Petioles thick, glabrous, 3 to $8 \mathrm{~mm}$. long; leaves entire, 10 to $15 \mathrm{~cm}$. long, 5 to $6 \mathrm{~cm}$. wille, acute, or very obscurely broadly-acuminate, tapering to the acute or rarely slightly obtuse base, shining and glabrous above except for few short scattered white hairs on the nerves, beneath with few scattered hairs on the nerves, and bearded in the axils with few or many usually brownish hairs, otherwise glabrous except for a very few scattered hairs on the reticulations. Nerves prominent, 5 or 6 pairs. Stipules deciduous, triangular, acuninate, 6 to $8 \mathrm{~mm}$. long, sparingly appressed-hirsute and ciliate on the margins, leaving a ciliate ring above the leaf sear when fallen. Inflorescence axillary, cymose rather densely, 6 to 8 flowered; peduncles glabrous, $15 \mathrm{~cm}$. long. Flowers sessile or nearly so. Calyx, purplish, glabrous, alout $3 \mathrm{~mm}$. long, truncite or obseurely and broadly 5-toothed. Corolla $1 \mathrm{~cm}$. long, 5-lobed, densely appressed-villong thronghout.

Island of Dinagat, $463,456$.

A species very closely related to Timonius compressicaule (Min.) (Polyphragmon compressicuule Miq.), from Sumatra, but differing in its much thicker purplish, glabrous, terete, ultimate branches, glabroum calyx, and longer corolla. 
IXORA Jinn.

I. coccinea Linn. Sp. P'I. 110. 1753.

Mindanat, l'rov: '/amboanga, 650.

Malayan Peninsula and Archipelago ete.

*I. amboinica DC. Prodr. 4: 487. 1830.

Luzon, Prov. Camarines Sur, Pasacao, 820.

Aunboina.

* I. timorénsis Decne. Noux. Aun. Mns. Par. 3: 418. 1834.

Luzon, Prov. Camarines Sur, Pasacao, 246.

Timor to Australia.

I. spu. indet. Several other distinct species are represented in the collection, but I an wnable to idcentify them at present, 170, 233, 243, 423, 480, 807.

PSYCHOTRIA Linn.

P. malayana Jack. in Malay. Misc. 1: 3. 1820.

Luzon, I'rov. Camarines Sur, Pasacao, 196, a broad-leafed form. Mindanan, Prov. Surigao, 472.

Malayan Peninsula and Archipelago.

P. sple indet.

Mindanao, 321.

\section{MORINDA Linn.}

M. bracteata Roxb. Hort. Beng. 15. 1814. (M. liguluta Blanco.)

I uzon, Manila, 731; Pror. Bataan, Mariveles, 766; Prov. Camarines Sur, Pasacao, 92. Mindanao, Prov. Surigao, 304, 411.

Southern Asia, Malayan region, etc.

M. citrifolia Linn. Sp. Pl. 176. 1753.

Luzon, Prov. Camarines Sur, Pasacao, 211. Mindanao, Pror. Surigao, 679.

Tagalog, Nino or Timbon ciso.

Sonthern $A$ sia to Australia.

M. tinctoria Roxb. ? Hort. Beng. 15. 1814.

Mindanao, I'rov. Surigao, 384.

Southern Asia and the Malayan region.

\section{GOODENIACEA.}

SCAEVOLA Linn.

S. kconigii Vahl. Symb. 3: 36. 1794. (S. lobelia Blanco, non Linn.)

Iuzon, Irov. Camarines Sur, Pasacao, 229.

A common plant along the seacoasts in the Tropics of the Oldl World.

COMPOSITA:

\section{MICROGLOSSA DC.}

M. volubilis DC. Prodr. 5:320, 1836.

Mindanao, 1'rov. Davao, 851.

Tropical Asia, the Malayan region, ete. 


\section{METHOD OF WORK ON THE FOREST FLORA DF JAVA.}

The following report on Dr. Koorders's method of studying the forest flora of Java is the first note regarding his successful methods that has been published in the English language. The manuscript of the present article was carefully examined by Dr. Koorders before I left Buitenzorg. Dr. Koorders has published several articles on his method of work in "Teysmannia," a periodical published in Batavia, but as his articles are in the Dutch language, and this periodical is accessible to but few American forestry students, it is hoped that the present article will prove of importance to those Americans who are interested in the subject of forestry, and especially to the workers on the problems presented by Philippine forestry conditions, which in many respects resemble the conditions in Java.

Manifestly, the method of conducting forest flora investigations outlined below has little or no bearing on the forestry ennditions in a temperate country like the United States, where extensive areas exist practieally entirely oceupied by a single species and where the individual tree species are few in number. But in a tropical country like the Philippines, where there are to be found no large areas occupied exclusively by a single species and where the number of tree species is very Iargely developed, it is evident that some special method of condueting the forest flora investigations must be adopted. The forester and lumberman must eacl take into consideration all the trees in the area in which he is working, whether or not they are of economic importance. It is of the utmost importance also that accurate information be compiled to enable us to prepare correct guides for the identification of the more important trees in the Archipelago.

While it may prove impossible to apply Dr. Koorders's method as a whole to Philippine conditions, yet certain features at least can be adrantageously applied here.

After rcceiving his appointment in the year 1888 to conduct investigations on the forest flora of Java, Dr. Koorders spent several years traveling in all parts of the island, selecting and studying the type vegetative areas, and collecting data and botanical material for working up the forest flora of the island. Since $\mathbf{1 8 8 8}$ the numbered botanieal collection of the herbarium of the Java Forestry Bureau, or the Herbarium Konrdersianum, has reached a total of about forty thousand numbers, 
and on this material is based the published results of these careful investigations, entitled "Bijlragen tot de kennis der Boonssoorten van Java," by Drs. Koorders and Valeton. These "Bijdragen" are issued in the Garden publication entitled "Nededeelingen uit's Lands Plantentuin," the first of these works on the forest flora of Java being publisher in 1896. Since that date seven numbers of the "Bijdragen" have been issued, of from two hundred to four hundred and seventy pages each, and at present two or three additional volumes are in the hands of the printer. In these "Bijdragen" the forest flora of Java is treated by families, with analytical keys to the genera. They contain careful generic and specific descriptions in both Latin and Dutch, with rather full citations and synonymy, followed by a long discussion of each species in the Dutch language, giving the detailed geographical distribution; the alsundance or scarcity of the species in Java; its general distribution outside of Java; habitat; peculiarities of the plant; time of flowering and fruiting; time of falling of the leaves, if a deciduous tree; local names; economic uses, etc. These "Bijdragen" constitute one of the most eareful and complete publications in any language on the forest flora of a tropical country, and much of the data on which this most excellent publication was based could have been secured in no other way than by the careful preliminary work on the type vegetative areas carried out by I)r. lioorders.

After a preliminary survey of the field, Dr. Koorders found it quite impossible for any one inan to make a complete study of all the forest regions of the Island, and accordingly, after much study on the subject, he worked out in detail the plan outlined below.

The principle of the scheme adopted was to select certain areas representing vegetative conditions in the various parts of the Island, typical both from a systematic and ecological standpoint; to number carefully the different species found in each one of the type areas, and to prepare full notes on each species, giving size, native names, economic uses, its distribution within the selected area, whether common or rare, epiphytic or parasytic, the character of the soil, etc., together with complete botanical specimens. When the flora of several of these type areas had been determined by Dr. Koorders, a hurried botanical survey was made in the country between two or more of the selected areas, to test whether or not the selected areas represented the typical forest flora of the region. It is needless to state that these areas were selected with great care, localities being chosen sometimes beeause they represented a particular type of forest, young jungle, old jungle, open lands with scattered forest vegetation, swamp forests, "djati" (teak) forests, etc., and sometimes because of certain geographical features.

In the many "test" botanical surveys that have been made in the country between these selected type areas, the wisdom of the original se- 
lection has in all cases been amply proved, because it has been found that the selected areas contained representatives of practically all the tree species that are found in Java. Oceasionally, however, there will be found outside the type areas a rare species or one of very local distribution that is not to be found in any of the selected type areas. In another manner the value of this method of studying the forest flora is shown, for by the eareful study of these type areas many tree species have been added to the Java flora that were previously unknown to the Island, and in some cases these speeies new to the flora are found to be of wirle distribution there.

Since 1888 some twenty-seven of these type areas have ben selected and carefully studied, many of them, especially those of the plain region, containing several square miles each; others, especially those on the high mountains, being of comparatively small size. They are not reserved from timber or agrieultural exploitation, and oceasionally it has happened that where type areas were thickly forested eight or ten years ago, now all of the original vegetation has been removed, and plantations of coffee, tea, or tobacco are found. It is not necessary to continue these extensive observations for a long period of years on all these type areas, and aecordingly, after five, six or eight years, as the case may be, when all the information available or desired has been secured, the labels on the trees are removed and the area is abandoned.

To establish a basis for these investigations, I)r. Koorders divided Java into three geographieal divisions, East Java, Middle Java, and West Java, each region characterized by extensive areas of country at a comparatively low elevation above the sea and cach containing high mountains approximating 4,000 meters in altitude. Again, he arbitrarily divided the Island into four altitudinal regions or sections, thus making in all twelve botanical regions in the whole Island. 'T'he first of these altitudinal sections extends from the sea level to a height of 1,000 meters; the second from 1,000 to 2,000 meters; the third from 2,000 to 3,000 meters, and the fourth ahove 3,000 meters. In each of these altitudinal sections in East, Middle, and West Java type areas were selected. In the first altitudinal section-that is, from the sea level up to 1,000 meters-is of course to be found not only the greatest superfieial area but also the greatest number of tree species, and acordingly in the first section, in Wast, Middle, and West Java, were selected three or four type areas ; in the second altitudinal seetion two or three type areas; in the third one or two type areas, and in the fourth but one small plot near the summit of some one of the higher mountuins in each of the three gengraphical regions.

In the Island of Java there are about 1,400 tree species known, and of these 1,400 species probably 1,200 attain a diameter of 12 centimeters or more. As noted above, the preponderance of tree species is found at the 
lower altitudes-that is, up to 1,000 meters above the sea. In this first altitudinal section there are found in the neighborhood of 900 distinct tree species; in the second there is a very perceptible decrease in the number of individual tree species; in the third this decrease becomes very strongly marked; and in the fourth or alpine region but fifteen or twenty tree species are found, and these much stunted in growth.

When a type area is selected for study, a birouac is established and a careful preliminary survey is made. After a thorough examination of the proposed area, the work of numbering the trees is commenced, and as each tree is numbered a printed slip or field label, with blank spaces, is filled out and afterwards filed with the botanical specimen, giving all the information obtainable regarding the tree, its name, economic uses, claracter of the bark, wood, ete. Any peculiarities regarding habit, foliage, flowers, or fruit, and the height and dianeter are, whenever possible, carefully measured. Careful, and if possible complete, botanical specimens are prepared at the same time, bearing the distinctive number and plot letter of the tree, and these botanical sjecimens, together with the notes, are carefully preserved in the museum for further study. At the time the trees are numbered a sketch map is also made showing the position of the bivouac, the inspection paths, and the position of each numbered tree with its distinctive number and letter, and further, a eareful catalogue is compiled, giving, first, the distinctive number and letter of each species; second, the native and Latin names, if known; third, the height of the tree in meters; fourth, its diameter in centimeters, measured at a height of 1.33 neters above the ground; and, fifth, a description of the exact locality of each labeled tree.

FORM OF IREGISTER FOR A TYPE AREA.

Register of the trees and other plants which have been labeled for the forest flora investigations in the type vegetation area, .................. Island of

Province of town of

Plot No. 1.-Altitude 1 to 300 meters.

[N me of any distinetive geographical feature-mountain, valley, river, lake, ete.]

\begin{tabular}{|c|c|c|c|c|c|}
\hline $\begin{array}{l}\text { Tree } \\
\text { No. }\end{array}$ & $\begin{array}{c}\text { P'lot } \\
\text { letter. }\end{array}$ & $\begin{array}{l}\text { Name of the } \\
\text { plant. }\end{array}$ & $\begin{array}{l}\text { Height } \\
\text { in } \\
\text { meters. }\end{array}$ & $\begin{array}{c}\text { Diam. } \\
\text { in } \\
\mathrm{cm} .\end{array}$ & $\begin{array}{l}\text { Deseription of the exnet locality of each num- } \\
\text { bered plant. }\end{array}$ \\
\hline 200 & f & $\begin{array}{l}\text { Putud } \\
\text { (Barringtonia } \\
\text { rucemosa.) } \\
\text { Moluve } \\
\text { (Vitex littora- } \\
\quad \text { lis.) }\end{array}$ & 10 & 10 & $\begin{array}{l}\text { 1)irectly back of the bivouac; solitary on bauk } \\
\text { of rivulet. } \\
\text { Six metcrs south of No. } 2001 \mathrm{f} \text {. }\end{array}$ \\
\hline
\end{tabular}


Field label used by Dr. Koorders when making general collections outside of the type vegetative areas, and from unnumbered specimens in the type areas.

[Translation, E. D. M.]

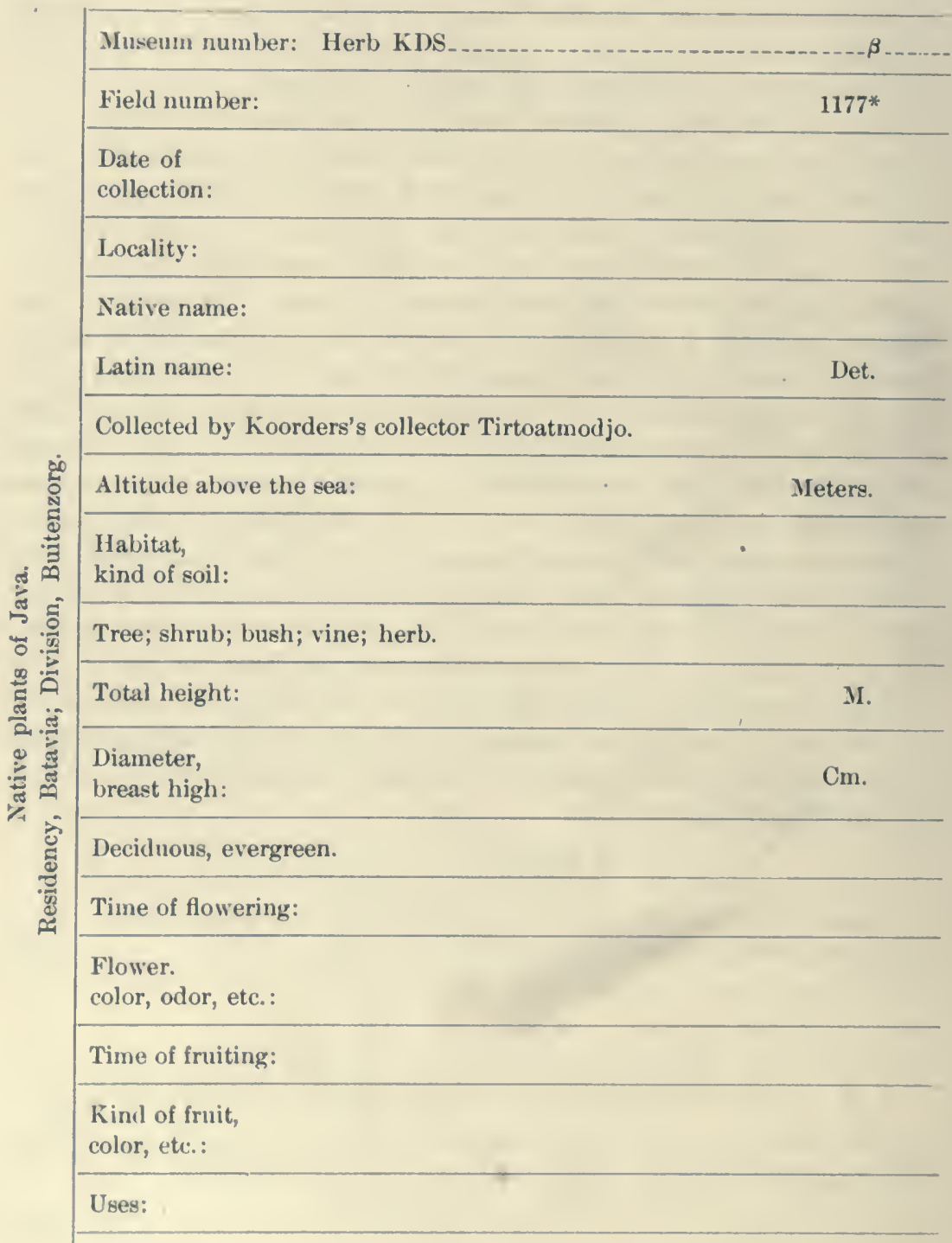

Habit like:

At this place. 


\section{1}

RFV EIRS:

Trunk:

Crown:

Bark:

Sapwood:

Heartwood:

Mature leaves: 
Field label used by Dr. Koorders when securing material and data from the type reget:tive areas.

[Translation, E. D. M.]

NATIVE NAME Jas. Marl. Sund.

Lat. name.

HEIGHT:

(To the top. in meters) . measured; estimated.

DIAMETER: (Breast high, in centimeters) measured; estimated.

1. Straight or colunnar; slightly bent; much bent.

2. cylindrical flattened angular twisted.

TRUNK:

3. Knurls (with - without); channels (with without); thorns (with without).

4. Buttresses (with without); aerial roots (with ............ without).

5. First branches \pm -..-_-_-_-- meters from the ground.

1. $\frac{1}{2}, \frac{1}{3}, \frac{1}{4}$, ete ............. of the total height.

CROWN:

2. Irregular; spherical; semispherical ; egg-shaped; cone shaped; flat; troad; small.

3. -...-...-. thin; medium; thick.

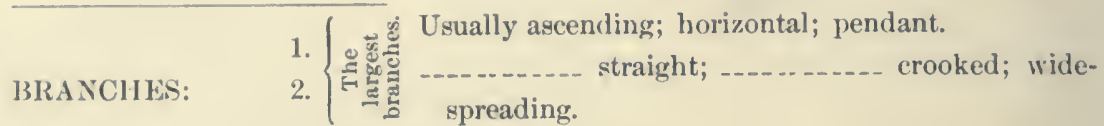

3. Ultimate twigs

1. Thickness in $\mathrm{mm}$ measured; estimated.

2.

fough brittle hard.

3. $\begin{cases}\text { is } & \text { Outside bark } \\ 0 & \text { Middle bark } \\ 0 & \text { Inner bark }\end{cases}$

4. smooth; usually rough; not; little; very scaly.

BARK:

5. Bast; with vertical horizontal

6. Lenticels; with without.

7. Milky sap; with without. resin, gum, etc.

8. Chlorophyll; with withont; with

9. Odor; with much; little; without.

10. Taste; with without. without.

FI.OWER BCIS: 1. Color, outside

1. Color of whole inflorescence

2. Odor

3. Taste

FI.OWERS:

4. Color of the flower parts:

Calyx

Corolla ...........-

Stamens: filamients anthers

Pistil $\left\{\begin{array}{l}\text { Stigma } \\ \text { Style } \ldots . . . . .- \\ \text { Ovary }\end{array}\right.$ 
RFIVRRSE.

1. Color: outside

IOUNG FRUIT: 2. Odor: (crushed) with without.

3. Taste: with without.

1. Dry or fleshy; dehiscent or indehiscent.

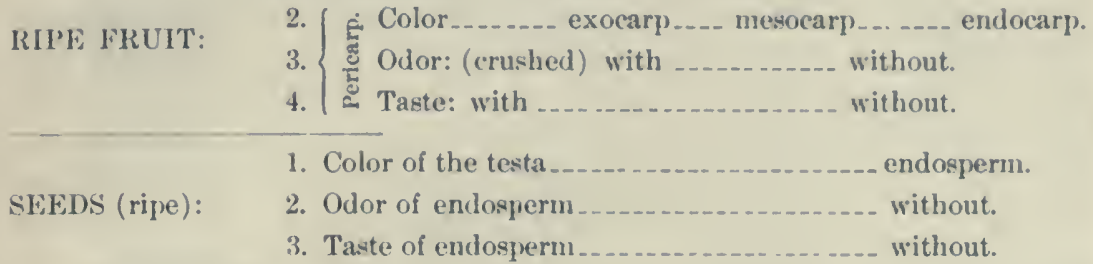

1. Color: above_............... green; leneath ....... green.

YOUNG LEAVES:

2. - .

3. Odor: (crushed); with ................... ; without.

4. Taste: with _.................. without.

1. Color: above ............... green; beneath _...... green.

OLD LEAVES:

2. shining dull.

3. Odor: (crushed); with

4. Taste: with _............... ; without.

HABIT: Resembles (compared with some well-known species.)

USES: Wood; bark; sap; etc

SAPWOOD:

HEARTWOOD:

\section{ROOTS:}


In labeling the trees some precautions must be observed. Zine labels, which would for most purposes be the best, are quite out of the question, as this, like all other metals, is greatly prized by the natives; and if it were used, the greater number of the labels would soon be destroyed by them. Accordingly, picces of board, about $30 \mathrm{~cm}$. square, are used. They are painted white, with the number and letter of the tree in black. A label of fair size is essential in order that it may be readily detected when one is searching for the tree the second time. 'These labels are nailed firmly to the trees with heary spikes, for if smaller nails were used, being easily extracted, they would prove too attractive to the natives. Even when the large spikes are used the natives sometimes suceed in extracting them. However, as the tree is carefully located, both in the eatalogue of the area and on the sketch map, little trouble is experienced in finding it the second time, even if the label has been removed. As a safeguard, however, a tree, as a rule, receives two distinct labels; one large and prominent, the other more inconspicuous.

With as many as twenty-seven type areas from which extensive botanical collections have been made, it is at once evident that some special method of labeling not only the trees but also the herbarium specimens and notes must be adopted in order to avoid confusion. Dr. Koorders has adopted the plan of combining a letter and a number, each altitudinal section having reserved for it alone a sequence of numbers between certain limits, and each type area in that section having a distinctive letter. Such a combination of letter and number shows at once not only the altitudinal and geographical section in which a plant was collected, but also the type area and the number of the tree in that area, and no other specimen in all the other twenty-six type areas has this same combination. 'To illustrate this point it is well to go into detail regarding this system of recording. As noted above, the great preponderance of tree species is found in the first altitudinal section, that is, below 1,000 meters. Accordingly there are reserved for the seleeted type areas in this section the numhers from 1 to 3000 , and these numbers are used in the first altitudinal section in all the geographical regions, but each geographical region has a distinctive series of letters.

Any number between the limits of 1 and 3000 shows at once that the specimen was collected from a numbered tree in the first altitudinal seetion, and the letter used in combination with the number cited shows the exact type area and the geographical region in which the area is situated. It is, of course, not possible to find 3,000 different species in any one type area, but this large number is reserved because it is often necessary to label several specimens of the same species; $\sigma^{\top}$ and 9 plants of the same species; old and young trees of the same species, etc. Possibly three or four, or even five or six, type areas may be reserved in the first altitudinal section 
PLATE VII.
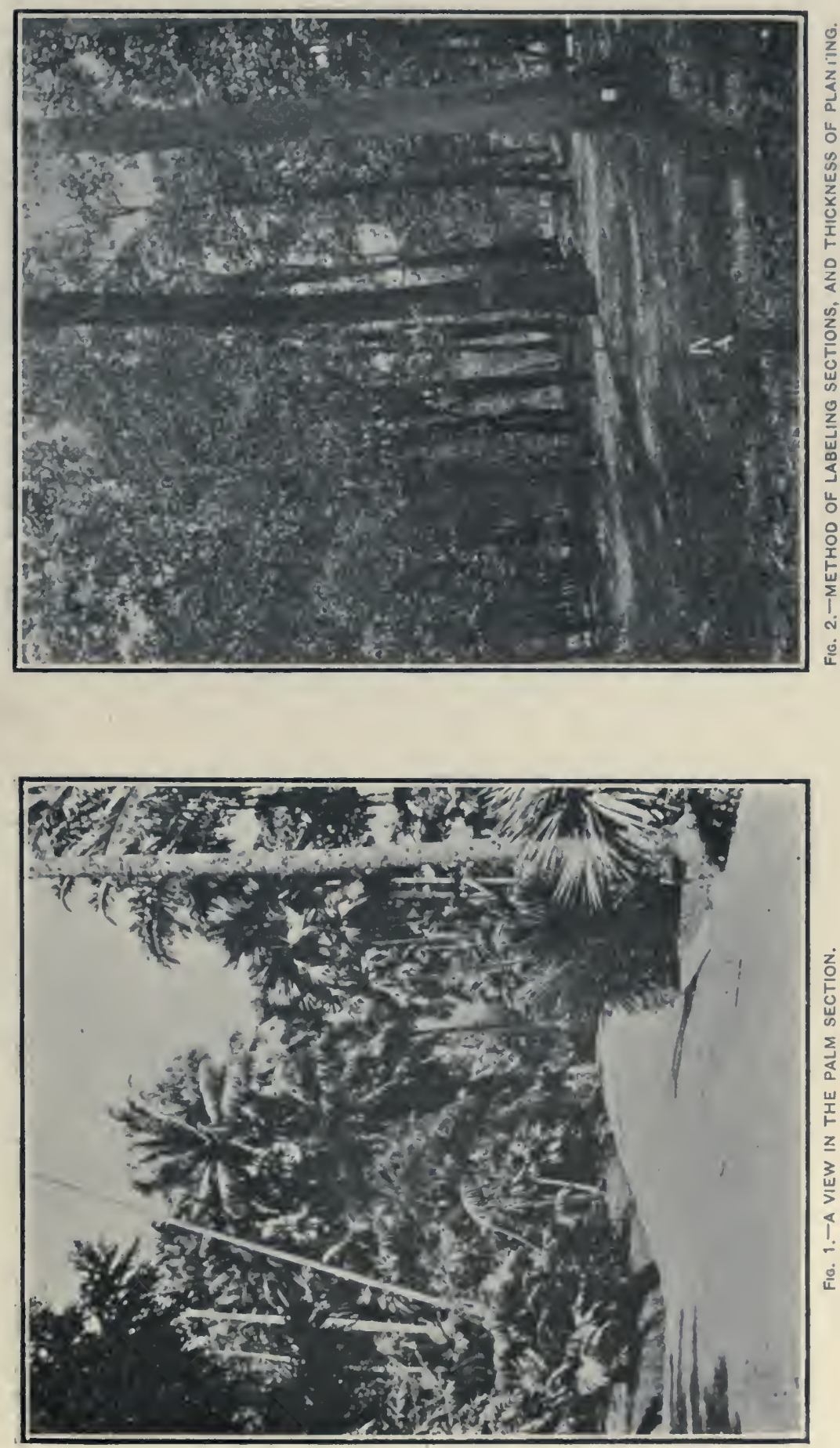

PLATE VIII.

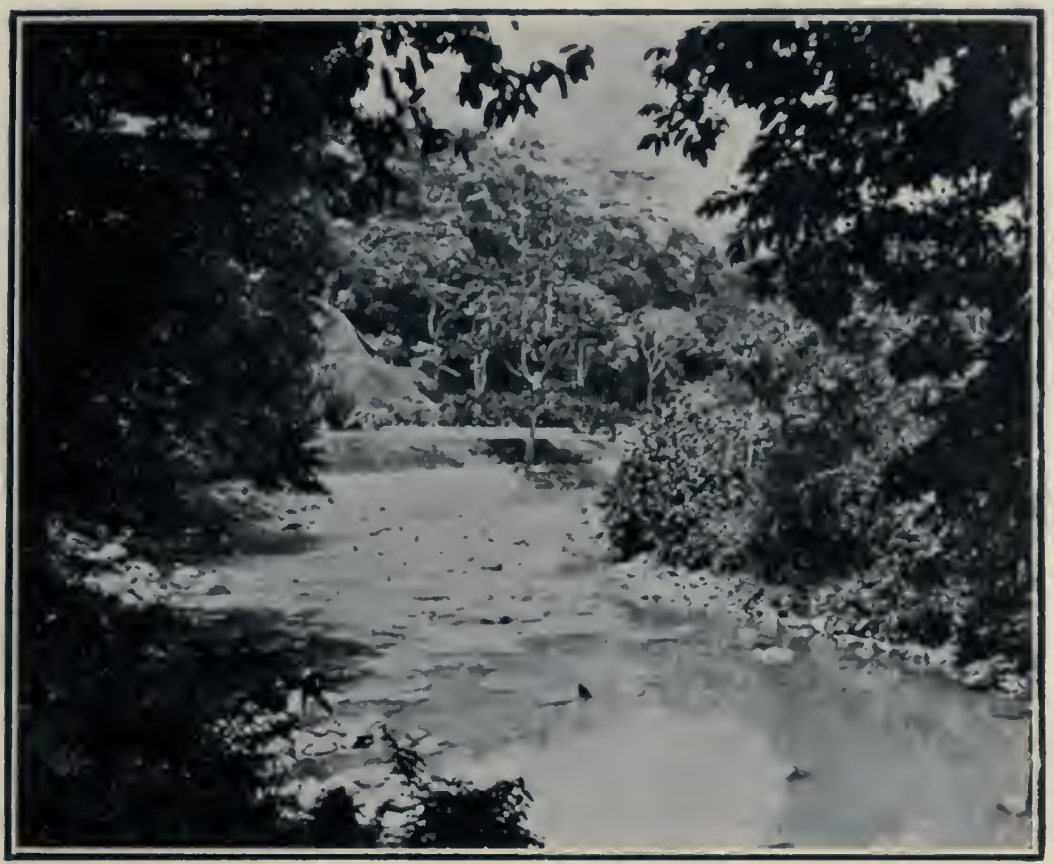

FIG. 1. -THE RIVER IN THE GARDEN.

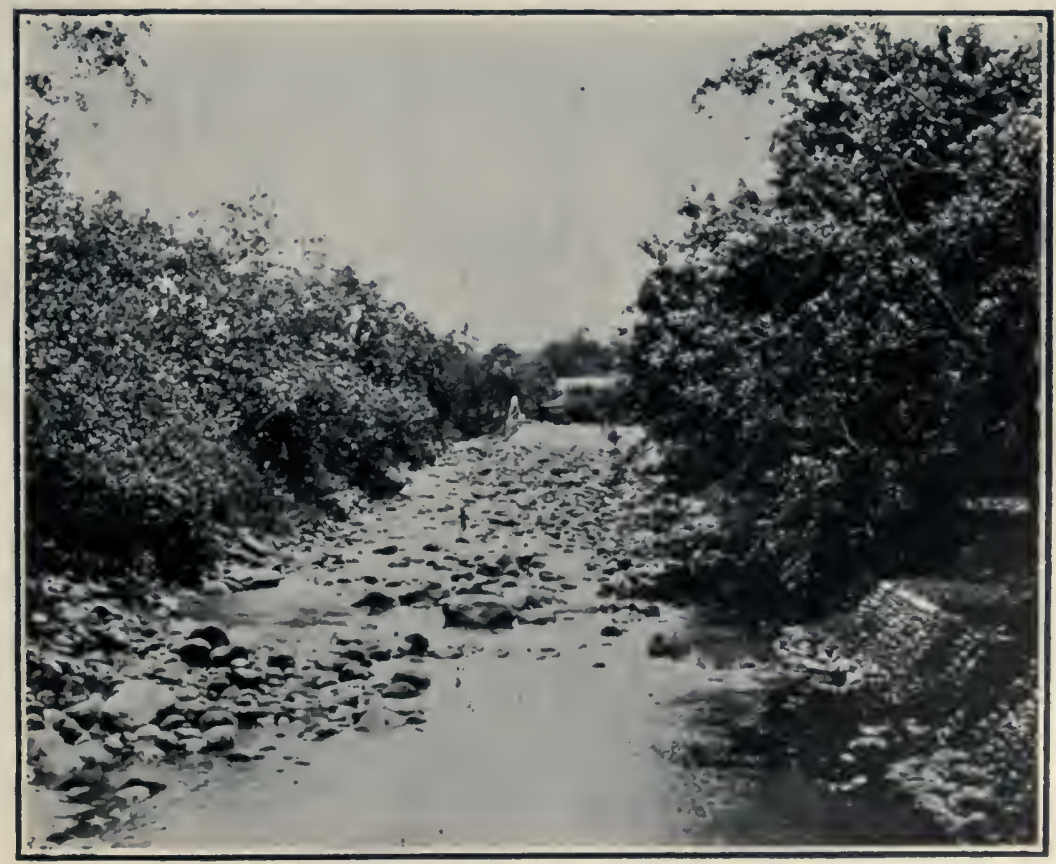

FIG. 2.-THE RIVER IN THE GARDEN. 

in the geographical region of East Java, the same number in the corresponding altitudinal seetion in Middle and also in West Java. For the geographical region of liast Java the letters $a, b, c, d, c, f$ are reserved; for the geographieal region of Middle Java the letters $g, h, i, j, k, l$ are reserved, and for the geographieal region of West Java the letters $m, n, p$, $t, r, s, w$ are reserved.

In Last Java the first type area established in the first altitudinal seetion receives the letter $a$, and the first plant numbered in that seetion receives the number 1 in combination with the letter-that is, $1 a$; the second plant numbered is $2 a$; the third $3 u$, and so on up to 3000 , if neessary or desirable. The seeond plot in the same altitudinal section and geographical region may be established 100 miles away from the first, and this receives the distinctive letter $b$; the first numbered tree in that section is $1 b$, the second $2 b$, and so on. In the seeond altitudinal section there is a great decrease in the number of tree species, and for this section are reserved the numbers from 3001 to 5000 , used in combination with the same letters $a, b, c, d, e, f$ for East Java, as in the first altitudinal section, these six letters being the distinetive letters for the geographical region of East $J$ ava. As in the first altitudinal section, the first type area established receives the distinctive letter $a$, but the first tree that receives a number in that type area is numbered $3001 a$; the second $3002 a$, ete. The third altitudinal seetion is marked by a very sharp deerease in the number of tree speeies, and for this section-that is, between 2,000 and 3,000 meters in altitude-it is only necessary to reserve 500 numbers, the letters $a, b, c$, cte., being applied to the type areas established but the sequence of numbers employed being between the limits of 5001 and 5500 . 'The fourth altitudinal seetion, or the alpine region, is confined to the high mountain tops above 3,000 meters in altitude. 'T'he area of this high region is very limited, as is also the number of tree speeies, but fifteen or so being found. For this region only the numbers between 5501 and 5550 are reserved.

In Middle Java and in West Java, the other two geographical regions, the same sequence of numbers is used for the different altitudinal sections as in East Java, but Middle Java and West Java have distinetive series of letters as previously noted.

To further illustrate this scheme it may be well to eite and explain a few of the combinations. For instance, $1500 \mathrm{~b}$ shows that the specimen in question was collected between the sea lerel and an altitude of 1,000 meters in type area $b$, in the geographical region of Fast . Tava, and that further details regarding the specimen will be found in the register of type area $b$ and in the notes under that number. No. $3601 b$ shows at once that the specimen in question was collected at an altitude of between 1,000 and 2,000 meters in the geographical region of Middle $6425-5$ 
Java, in type area $l$. No. $5015 p$ shows that the specimen was collected at an altitude of between 1,000 and 2,000 meters in the geographical region of West Java in type area $p$. It is needless to state that these type areas are most carefully localized geographically so that the citation of the number and letter shows at onee the exact geographical locality.

For each of the twenty-seren type areas that have been studied in Java - a separate catalogue has been prepared. Each catalogue contains a sketch map of the type area, a short deseription of the reserved plot, its exact geographical location, exact altitudes of the more prominent geographical features in the area, the numbers and data regarding all the trees recorded, etc. When the trees are originally numbered it very often happens that a specimen may have neither flower nor fruit, one or both of which are generally essential for purposes of accurate identification, and hence it is often necessary to visit the type area again in three or six months to secure the necessary herbarium material. A competent native collector can be used for this purpose, and the original collection or "controlling material" that was secured when the tree in question was numbered is an exact check on the accuracy of the second collection.

In collecting botanical material at the time the trees in one of the type areas are being numbered, it often happens that it is desirable to prepare botanical specimens of a certain species, and at the same time it is not advisable to number the specimen in question, especially with small shrubs, herbaceous plants, grasses, etc. In this case the specimen receives a field number, but of a sequence not reserved for any of the type areas, and the number of such a specimen is always followed by an asterisk, which shows at once that the herbarium specimen in question was not secured from a numbered tree.

With large and separately numbered collections from each of the twentyseven type areas that have been studied in Java, not to mention the great amount of botanical material that has been collected outside of the type areas, it is very evident that some special method of recording must be devised. At Buitenzorg, when a collection is brought in from the field, the field number is reserved on the original specimen and entered in a special column in the museum eatalogue. At the same time each specimen receives a new number and letter, the permanent herbarium or museum number which is written on the label with red ink and which is entirely independent of the field number. This permanent or herbarium number is the one under which the specimen is permanently entered in the herbarium eatalogue, is the only number ever referred to in publications, and is the only number used in the distribution of duplicates, ete. 
SAMPLE PAGE.

Calulogue of the herburium.

\begin{tabular}{|c|c|c|c|c|c|}
\hline $\begin{array}{l}\text { Museum. } \\
\text { No. }\end{array}$ & Latin name. & Fautly. & $\begin{array}{l}\text { Collec- } \\
\text { tion } \\
\text { No. }\end{array}$ & Local name. & $\begin{array}{l}\text { Not only } \\
\text { Jn lier- } \\
\text { barlum } \\
\text { bnt also- }\end{array}$ \\
\hline $15401 \beta$ & Lagerstromia speciosa Pers._... & Lyth & $432 \rho$ & Woengel - & A. \\
\hline $15402 \beta$ & Peinphis acidula Forst _...- & Lyth $\ldots$ & $1050 \beta$ & Sentigi ... & $\ldots . . .-10$ \\
\hline $15403 \beta$ & Barringtonia racemosa $\mid \mathrm{Bl} \ldots \ldots$ & Myrt $\ldots$ & $250 l$ & Pintaid.... & 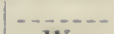 \\
\hline $15404 \beta$ & 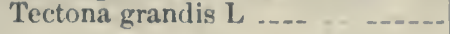 & Verb ... & $16 \leadsto$ & Djati ...- & W. \\
\hline
\end{tabular}

In this catalogue the first column contains the permianent herbarium number, in combination with the Greck letter Beta, which is the initial letter of the old Javanese name of Buitenzorg, i. e., Bogor. 'The second column contains the Latin name of the plant; the third its family, generally abbreviated; the fourth, the field number of the plant; the fifth its local name; while the sixtl is reserved to note.whether or not material other than dry herbarium specimens was preserved; A.=alcoholic material; $\mathrm{B} .=$ bark; $\mathrm{W} .=$ wood, etc.

At the end of one or two, or at most tliree years after a type area has been selected and the trees numbered, it is revisited and careful notes are made of the changes that have taken place in the time clapsed. A second set of botanical specimens is also prepared at this time. The type areas are visited as often and as many times as may be necessary to secure the information available or desired.

This careful method of working out the forest flora under tropical conditions not only furnishes the most accurate information concerning the geographical distribution and scarcity or abundance of the many individual species but makes possible a most careful check on the accuracy and value of the many native names, and on the economic uses of the different species. Often it is found that "floating" names exist-that is, the same name is affixed to several different species, or to groups of related speciesand on the other hand, names applied to certain species, especially to those of economic importance, will be found to be quite invariably applicd to the same species. By preparing such a series of careful notes on the many spccies, and by checking over the local names each time a type area is visited, one can soon readily determine which of the native names are trustworthy and which are doubtful and should be discarded.

In the Philippine Archipelago, extending as it does over about fifteen degrees of latitude, there are probably 1,500 distinct species that would be classed in the forest flora; and of these 1,500 species some 75 or 80 per cent attain a diameter of ten or more centimeters. With this exceedingly large number of individual species, in order to obtain any definite knowledge of the component species of the Philippines forest flora, the extent of the forest areas, prevalence of valuable economic species, etc., it is quite 
necessary to adopt some definite plan of investigation. 'T'o me the plan outlined above appears to be the most practicable one, and the one that will be productive of the best results.

As Java was divided by Dr. Koorders into three geographical regions from east to west, so, at his suggestion, the Philippine Archipclago can be divided into preferably three geographical regions, from north to south. In this preliminary report 1 would recommend that the Archipelago be divided into the following regions for the study of the forest flora: First, the northern or Luzon region, to consist of the Islands of Luzon, Mindoro, and Marinduque, with their outlying smaller islands; second, the eentral ol Samar-Paragua region, to consist of the Islands of 'Samar, Leyte, Masbate, Bohol, Cebu, Negros, Guimaras, Panay, Calamianes, Paragua, ete; third, the southern or Mindanao region, to consist of the Island of Mindanao, the Sulu Archipelago, and the outlying sinaller islands.

As in the Philippines we have no mountains equalling those of Java in altitude, it will be more convenient to distinguish but three altitudinal seetions rather than four, as in the investigations in Java. First, from the sea lerel to 1,000 meters in altitude; second, from 1,000 to 2,000 meters in altitude; and third, all mountain tops above 2,000 meters.

For the Luzon region the letters $a, b, c, d, e, f$ can be reserved; for the Samar-Paragua region the letters $g, h, i, j, k, l$, and for the Mindanao region the letters $m, n, p, r, s, t, w$.

For the first altitudinal section the sequence of numbers between 1 and 3000 can be reserved; for the second altitudinal region the numbers between 3001 and 5000 , and for the third region, or all altitudes above 2,000 meters, the numbers from 5001 to 5500 .

Proposed Lotmical resgions for the purpose of studying the forest flora of the Philippine Archipelngo.

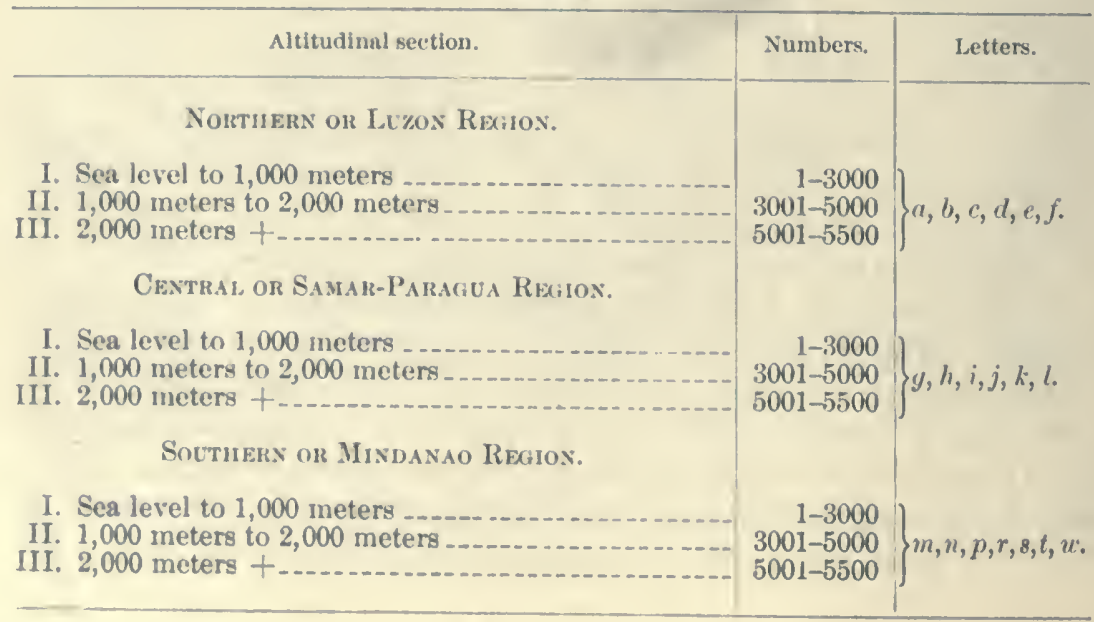


Sketch showing the proposed geographical regions and altitudinal sections for the study of the foreat floru of the Philippine Archipelngo.

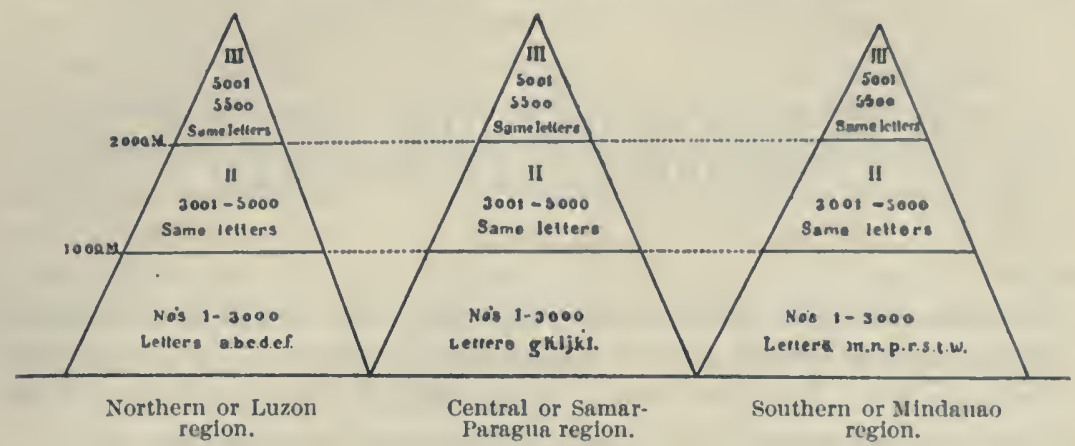

In applying this plan of work to the Philippine conditions, it is only necessary to use the combination of the type area letter and the tree nunber, as illustrated above in the detailed report on Dr. Koorders's method of work in Java. This method of studying tropical forests is, I believe, the most practicable, productive of the best results both from an economic and a scientific standpoint, and in the long run probably the most economical which can be adopted in the Philippines. It has been found entirely satisfactory after fourteen years of application in the Island of Java, where the forest conditions are very similar to those of the Philippines.

In closing, I strongly urge that this system be given a thorough trial in the Philippines, by establishing several of these type areas in the near future. Preferably, the first type area should be near the city of Manila, in such a location that it can be easily reached from the city, and for this first type area I believe no better location ean be selected than Mariveles, in the Provinee of Bataan, as here a type area can be readily established, extending from the sea level to an altitude of about 1,000 meters. A type area so established will not only serve to give use aceurate information concerning the forest flora of the vicinity of Mariveles, which is typical of the whole Province of Bataan, but, in the absence of a proper botanical garden, will be of the greatest value in assisting employees of the Forestry Burean to become acquainted with the forest conditions in the Philippines, the characteristics of the more valuable timber trees, ete, and to identify these species in the forest. 


\section{THE BOTANICAL INSTITUTE ('S LANDS PLAN- TENTUIN) AT BUITENZORG.}

Several extensive publications describing this admirable institution have recently appeared, and it is accoldingly not my object to go into detail regarding it in the present paper, but only to record some of the methods of investigation and call attention to the equipment of the 's Lands Plantentuin.

The botanical garden at Buitenzorg forms a great park surrounding the residence of the governor-general of the Dutch East Indies. It was established in 1817 by Reinwardt, who was afterwards a professor at Leiden University. Following Reinwardt, Dr. C. L. Blume, as the first regularly appointed director, had charge of the gardens. In 1830 James Hooper, who came from the Royal Gardens at Kew, England, returned to Europe, and Mr. J. E. 'I'eysmann was appointed his successor. 'To him are largely due the present beauty and utility of the botanical garden. It was 'Teysmann who, at Hasskarl's suggestion, adopted the plan of arrangement after the natural order of plants, so that, owing to this most convenient arrangement, it is now comparatively easy for one to study and compare the species of any one natural order, although there are now in cultivation over 10,000 species, covering an area of 147 acres.

When Teysmann resigned in 1859 Binnendyk was appointed to succeed him, and in the meantime the whole institution was reorganized on a plan drawn up long before by Teysmann.

In 1868 Dr. Scheffer, a man well qualified for the position, was appointed director. In 1875 he established the publication of the "Annales du Jardin Botanique de Buitenzorg." Above all other things in value, however, is the experimental garden established by Scheffer in 1876 - a garden of 180 acres for experimenting on a large scale with economic species. Dr. Scheffer died in 1880 , and Dr. 'Treub, the present director, was appointed his successor.

The present magnificent equipment and wide influence of the 's Lands Plantentuin is due largely to the well-directed efforts of Dr. Treub, a gentleman who is by education and inclination most admirably fitted for directing such an institution. At the time of his appointment in 1880 the institution practically corresponded to what is now the fifth division; for at that date that most practical adjunct, the experimental garden, had been established but four years. Since the year 1880 the institution has 
extended its field from almost pure botanical work, to investigations which at the present time eover all lines of tropical botany and agrienlture. Thorough work is the watehword; and the suceess of the many investigations carried out at Buitenzorg have had great influence not only on the agrienltural development of the Duteh East Indies, but throughout the tropies. The suceess of the investigations made at this institution is recognized by the Duteh Government, as is shown by the large appropriations made for its maintenance and for special investigations, construction of new buildings, etc. It is also interesting to note here the interest that the Dutch planters take in the institution; for the entire expense of certain investigations is borne not by the Government but by an association of planters.

At the time of Dr. 'Treub's appointment as direetor in 1880 but two series of publications were issued; to-day there are six. In 1890 there were but four laboratories; to-day there are eight, each with most modern equipment. The working staff of the institution at present numbers about thirty Europeans and more than two hundred natives.

The institution known as "s Lands Plantentuin" consists of the following divisions:

I. IIerbarium and museum.

II. Botanical laboratories.

III. Agricultural and experimental gardens (181 acres), with laboratory for agricultural chemistry.

IV. P'harmacological laboratory.

V. Botanical Garden (145 acres) and the Mountain Garden ( 77 acres, and 700 acres virgin forest), with laboratory.

VI. Office, library, and photographic laboratory.

VII. Forest flora collections.

VIII. Laboratory for the study of Deli tobacco.

IX. Experimental station for coffee.

X. Laboratory for agricultural zoology.

Each of these divisions has eommodious and well-equipped buildings and laboratories. The second division has a special laboratory reserved for foreign scientists, where many avail themselves of this excellent opportunity for studying tropical botany.

The Botanical Garden, from a climatic standpoint, is admirably situated, for under tropical conditions it is neeessary, in order to have a successful garden, to have it situated in a locality where the rainfall is distributed throughout the year instead of having the two well-marked seasons-wet and dry. At Buitenzorg this condition is fulfilled, for during the so-called dry season rains are frequent, while during the rainy season one can expect rain every day-always in the afternoon at about 4 or 5 o'clock. The average preeipitation in a year is about $4,680 \mathrm{~mm}$, while sometimes as much as $160 \mathrm{mmi}$. will fall in twenty-four hours, as happened once or twice during my stay at Buitenzorg. 
'The experimental garden consists of 180 acres, and is situated about two miles from the Botanical Garden proper. The object of this garden is to grow species of economic importance on a large scale; to supply material for investigations made in the various laboratories; to experiment with various methods of cultivation, treatment, etc. Practically all the tropical plants of great economic importance are found here, each species being represented by a large number of specimens. The species cultivated include many varieties of coffee, tea, and cacao; abacá and other fiber plants; pepper, nutnegs, cloves, and other spices; erythroxylon, cinchona, and other plants producing alkaloids; palms of ceonomic importance; grasses yielding essential oils; numerous varieties of gutta-percha, caoutchouc, and other gum-producing trees; plants producing dyes, tannin, eanıphor, etc. Also connected with this garden is a large area of paddy land for experimenting in rice enlture.

\section{APPROPRIATIONS.}

For the support of the institution at Buitenzorg annual appropriations are made by the Government. Several appropriations for one year (1897) are as follows:

Director

Division $I$.

Herbarium and muscum:

Chief (botanist) _......... 9,600

Curator _... 2, 400

12,000

Botanical laboratory:

Chief

Botanist for tobacco _.

Kixpenses _... 700

Dirision III.

Experimental garlen and laboratory for agricultural chemistry:

Chief

A wyistant chemist _._._._.

Assistant chemist for tea_..._._.

Special fund _..._. 600

Garlener ............

Iabor ............ 6,000

Dirision $I \mathrm{I}$.

Pharmacological liboratory:

Chief

Expenses ........................ 1,000

* One guilder $=\$ 0.40$ United States currency. 
Dirision 1".

Botanical Garden proper and Tijbodas garden:

Clrief

Gullders

Assistant chief

5,400

Gardener

2,400

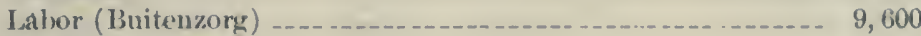

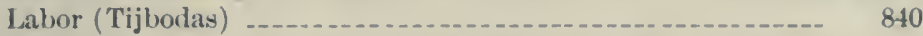

Ihirision $1 \%$.

Bureau, library, photography:

Chief _...

Photographer _.......... 3,600

Assistants _.................................................... 900

Labor _............. 2, 100

Books, equipment, ete_._._._._._._. 12, 000

Dirision IIII.

Forest flora:

Chief

Botanist _.

Traveling expenses, ete

Dirision VIII.

Laboratory for Deli tobacco:

Chief (botanist) -

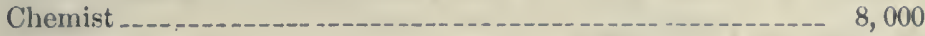

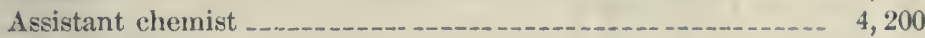

Assistant chemist _...................... 3, 000

Expenses _... 2,400

Division $I X$.

Laboratory for the study of coffee:

Chief

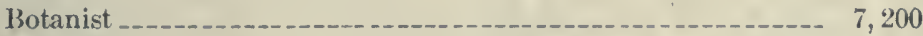

Expenses_._._. 5,700

Dicision $\mathrm{I}$.

Laboratory for agricultural zoology: $\quad$ Naturalist
Nat,

Expensey

Prublications.

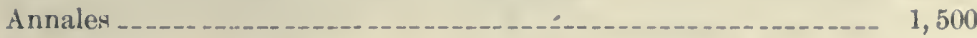

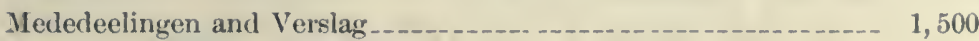

Construction and repairs.......... 10,000

Extraordinary expenses _..._.......

Total 


\section{PUBLICATIONS.}

1. Verslag.

2. Annales du Jardin Botanique de Buitenzorg.

3. Mededeelingen uit's Lands Plantentuin.

4. Icones Bogoriensis.

5. Bulletin de l'Institute Botanique de Buitenzorg.

6. Korte Berichten.

'I'he Verslag (Annual Report) dates from 1868, when it was but a fourpage pamphlet, giving a list of accessions to the garden, etc. 'The publication increased gradually until 1882, when the form was changed, and to the general report of progress, accessions, etc., was added the results of special investigations-for instance, "A study of gutta-percha," "A study of jute culture." This publication, the annual volumes of which average some two hundred and forty pages, is in the Dutch language.

The Annales is purely scientific in its character. Its publication was commeneed in 1876 and it has now reached its seventeenth volume. Articles in this publication must be in either French, German, or Englishcertainly a remarkable provision-and contributions are thus received from many scientists not comnected with the Buitenzorg institution. Since the establishment of this journal many important articles have appeared in its pages on systematic botany, physiology, morphology, ecology, and embryology. 'The publication is quite essential to all botanists who are working on the flora of the Indo-Malayan-Australian region, which includes the Philippines.

The publication of the Mededeclingen (Communications) was commenced in 1884, and has now reached to No. 56, the individual volumes extending from small numbers of 50 pages to volumes of over 700 pages. The greater part of this publication is in the Dutch language, although technical botanical descriptions are in Latin. A great variety of subjects is covered, in fact nearly all lines of agricultural investigations, chemistry, botany, etc. A few titles will serve to give an idea of this work: "Enquiry concerning the gutta-percha-producing trees of Pedang"; "The leaf disease of coffee"; seven volumes on "Contributions to the knowledge of the forest flora of Java"; "History of the garden"; "Nutmeg culture"; "The birds of Java"; publications on tobacco, tobacco soils, poisonous plants, etc.

'The Icones Bogoriensis consists of plates and descriptions of new, rare, or especially interesting plants. The publication of this serial, now in its fourtl volume, was commeneed in $189 \%$.

The Bulletin was first issued in 1898 and has now reached No. 15. This publication consists of technical articles on chemistry, botany, etc., published in German, French, or English. The size of the bulletin varies from 10 to 80 pages.

'The Korte Berichten (short notices) are printed in Teysmannia, a periodical published in Batavia, and not strictly a publication of the bo- 
tanical garden, yet the greater part of the articles that appear in its pages are written by attachés of the Buitenzorg institution.

In addition to the above serial publications many miscellaneous books and pamplilets have been published by the institution, among which are the following: "Catalogue of the Library," 370 pages, with yearly supplements since 1894; "Seventy-fifth Anniversary of the Establishment of the Garden," 1893, 426 pages; "Catalogue of the Phanerogams Cultivated in the Garden," 60 pages (incomplete); "Prodrome de la Flora Algologique des Indes Neerlandaises," 277 pages; "Hand Guide to the Botanical Gardens," 36 pages.

\section{LABORATORIES.}

For many years but one or two laboratories existed at Buitenzorg, and as late as $\mathbf{1 8 9 0}$ there were but four; to-day the following laboratories exist, all with modern equipment: Agricultural chemistry, pharmacology, agricultural zoology, vegetable pathology and physiology, botanical laboratory reserved for foreign investigators for personal work, laboratory for the study of tea, laboratory for the study of rice, and laboratory at Tjiboda for the study of the virgin forest. At present a large building is in course of construction to be devoted to agricultural chemistry.

\section{THE HERBARIUM.}

The herbarium, containing approximately two hundred thousand speciinens, consists of the old or great herbarium, which is deposited in the botanical museum; the new, or forest flora herbarium, which occupies a separate building at the present time, but eventually will be combined with the great herbarium; the "garden herbarium,"species cultivated in. the laboratory for the use of foreign scientists; and the "Herbarium Borgoriense," a collection of the plants growing in the vicinity of Buitenzorg.

The arrangement of the herbarium is strictly alphabetical under families, genera, and species-the system followed in publication being that of Bentham and Hooker. The old, or "great lierbarium," consists of plants collected between 1845 and 1858 by Zollinger; the rich eollections made by Teysmann, 1854 to $187 \%$, in all parts of the Archipelago; the specimens of Kurz, Scheffer, Hallier, Niewenhaus, Burck, Treub, Van Romburgh, and others, and a great quantity of duplicates from Calcutta, Kew, Singapore, Berlin, and Melbourne, representing the flora of British India, the Malayan Peninsula, New Guinea, Australia, and some material from North and South America, but practically none from the Philippines.

The museum contains about 1,500 specimens of dried fruits, 1,500 alcoholic specimens, 250 specimens of vegetable fibers, 200 specimens of gutta-percha, 200 specimens of caoutchouc, ete.

In the forest flora herbarium, which contains only specimens from Java and Maedura, a most careful record is kept of all collections, and 
for convenience but two series of numbers are used-one the "Herbarium Koordersianum" and one the "Herbarium variorum," the former now numbering from 1 to nearly 40000, the latter from 1 to about 500. Lach collection has a distinetive label. 'The method of building up the collection is as follows:

The "Herbarium Koordersianum."-Either Dr. Koorders himself collects the specimens or some native collector is sent out with the usual botanical equipment, and also a series of consecutively numbered field labels, always numbering from 1 to 500 , 1000, or 2000, according to the length of time the collector is to be ont. By providing these consecutively numbered labels, all chance of duplicating numbers in the field is avoided. 'The first specimen collected receives label No. 1, the second No. 2, ete. When the collection is brought in it receives in addition to the "field number," a museum number of the "Herbarium Koordersianum." The collector's number is preserved in the records, and on the herbarium speeimen, but the museum or "Herbarium Koordersianum" number is the only number used in the distribution of duplicates to other botanical institutions, and all reference to the specimens are made by the use of this latter number, which is entered in a register, with references to the collector and his field number. For example, at a certain date the "Herbarium Koordersianum" register numbers 15500 , and at this date a collection of 1,100 specimens is brought in by some field collector; No. 1 of this collection receives the new number, 15501, and so on until the new collection has been incorporated in the "Herbarium Koordersianum" series.

The "Herbarium variorum."-This is of course combined with the general forest flora herbarium, and is similarly numbered, but with a different series. For instance, some Army officer sends in 50 plants; these are numbered "Herbarium variorum" from 1 to 50. Later some civil official may send in 100 specimens; these are numbered from 51 to 150 , with, of course, the collector's name and date preserved. By this method a very careful record can be kept of all accessions.

The original field labels are pinned to the herbarium sheet, and all references, labels, and tags on the sheet receive the register number, either of the "Herbarium Koordersianum" or "variorum," as the case may be. These labels are not attached permanently because often it is of importance that they be removed when one is working up some genus or species, in order that he may have all the references to the genus or species in question before him at one time. By reference to the register and the herbarium number, the labels can readily be reattached to their proper sheets. The permanent labels are always pasted to the sheet on the lower right-hand corner, as in American herbaria.

In the general herbarium the specimens are mounted on paper of rather joor quality but of the standard size $-11 \frac{1}{2}$ by $16 \frac{1}{2}$ inches. The speci- 
mens are attached to the sheets by gummed strips of paper, while all labels are attached to the sheets by pins. 'This method of mounting plants is rather unsatisfactory, as with age the specimens become brittle and the leaves, flowers, and fruits of many of the specimens readily drop off and become lost. Labels attached with pins can not be considered permanent, as in the course of time the pins corrode, causing the paper to break and allowing the label to become detached. In the forest flora herbarium the specimens are attaclsed to the sheets by glue and in addition gummed strips, while the permanent labels are pasted to the sheet. I have instituted in Manila the latter method of mounting, and find it most pralctical and at the same time the most permanent method of preserving specimens.

Wooden herbarium eases are not used, but in both herbaria are hundreds of tin boxes about twenty inches long, twelve inches wide and seven inches deep. These boxes are arranged in suitable frame cases, those in the old herbarium extending entirely around the balcony or second floor of the museum building, those in the new herbarium in stacks extending in parallel rows in the herbarium room. The boxes open at the end, being hinged at the bottom, provided with a suitable fastener at the top and a proper label holder in front.

In preserving fleshy material alcohol has been found to be the only practical preservative for use in the Tropics. Experiments have been made here with various compounded preservatives containing alum, picric acid, etc., and also with formaldelyde, but all have proved unsatisfactory except alcohol. Alcoholic material is permanently preserved in glass bottles, museum jars, etc., but in the field zine collecting cases are always used. In collecting alcoholic material the fruits or flowers receive the field number of the herbarium specimens prepared from the same plants, and are then wrapped in thin paper and numbered again, in order to avoid errors, before they are placed in the alcohol; later the specimens receive the same museum numbers as the herbarium specimens.

Before the specimens are mounted they are poisoned by immersing for two or three minutes in a saturated alcoholic solution of corrosive sublimate. For this purpose a hollow log is used, the size of the trongh being about twenty inches long, thirteen inches wide, and eight inclies deep. Into this is poured the sublimate solution, and then the plants, each with a proper label, are placed on thin bamboo frames and immersed for the required time; then removed and placed in driers until thoronghly dry, then being mounted. In doing this work great care is exercised to avoid contact with the solution, the specimens being transferred from the bamboo frames to the driers by means of specially constructed bamboo implements. As each specimen is mounted, a small label is attached, giving the date of poisoning, initialed by the person who poisoned the specimen. 
COLLECTING.

Much of the botanical collecting is done by natives. For preserving alcoholic material in the field, a zine box 47 by 34 by $30 \mathrm{~cm}$., tightly fitted into a stout wooden chest, with suitable handles, hinged cover, and proper fastener, is used. The cover of the zinc box is in one piece, entirely removable, and when not in use this box is hermetically sealed with paraffin or other suitable material of a similar nature. In preserving alcoholic material there is first placed in the box a layer of cotton, common "capok" fiber (from Ceiba pentandra Gaertn), or other absorbent material, which is saturated with alcohol. On this are placed the paper packets containing the fruits or flowers to be preserved, each with its distinctive label and number. Another layer of cotton is then placed over them, then another layer of fruit, etc. The cotton is kept only saturated or partially saturated with alcohol. This manner of preserving is entirely satisfactory and far more convenient than the usual method of preserving in alcohol. Usually two or more collecting cases are taken, one for very fleshy fruits that would crush easily, and one for the more solid varieties.

For transporting dryer papers, species sheets, etc., tin boxes fitted into suitable stout wooden chests are used. These tin boxes are $66 \mathrm{~cm}$. long, $42 \mathrm{~cm}$. wide, and $28 \mathrm{~cm}$. high, and each will accommodate two stacks of the ordinary Chinese drying paper. In these boxes the driers and presses are placed at night, when the atmosphere is saturated with moisture, or during a rain. This protection of the drying paper is quite essential for the preparation and preservation of good botanical material under tropical conditions, as the drying paper and specimens very readily absorb atmospheric moisture.

Primarily, the 's Lands Plantentuin was a botanical garden pure and simple; and in its early history, although some attention was given to the introduction, cultivation, and dissemination of plants of economic value from other countries, its chief object was the study of systematic botany. As was the case with the many botanical gardens established by the English in the Tropics, this institution, as the flora of the country was worked up, gradually increased its work on economic lines, until to-day, although much attention is given to systematic botany, morphology, embryology, ecology, ete., the greater part of the work done is of an cconomic nature: the establishment, development, and maintenance of the botanical garden being simply a means to an end, namely, the application of seience to the economic agricultural questions of the day.

The great advantage of having practically all the investigations in agriculture, botany, chemistry, and allied sciences conducted by one institution, thereby avoiding the necessity of duplicating libraries, laboratories, and laboratory equipment, and obtaining the greatest results witl the least expenditure of money, is at once apparent. 
In the Island of Java there are two experiment stations for sugar; one in East and the other in West Java, and an experiment station for caeao; all of which are independent of the institution of Buitenzorg. 'The forestry bureau situated at Batavia and having charge of the Government forests is also an independent institution, although all of the botanical work of that office is done at the 's Lands Plantentuin by Drs. Koorders and Valeton. These independent investigations form only a very small part of the total investigations now being carried out in Java; all the rest of which are directly under the supervision of Dr. M. 'J'reub and his corps of able assistants, and under the auspices of the 's Isands Plantentuin at Buitenzorg.

I have here several acknowledgments to make-to Capt. George P. Ahern for the interest he has taken in identifying this material and in granting me authority to make the trip to Buitenzorg; to F. IamsonScribner, Chief of the Bureau of Agriculture, for allowing me so to arrange my work in his Office as to make it possible for me to make the trip; to Dr. P. van Romburgh, acting director of the 's Lands Plantentuin for granting me access to the library and herbarium of that institution; and to Dr. S. H. Koorders, in charge of the forest flora investigation in Java, for aid in identifying some of the more difficult and imperfect specimens, enumerated in Part II of this bulletin.

. Elmer D. Merrill, Botanist, Bureaus of Agriculture and Forestry. 

PLATE IX.

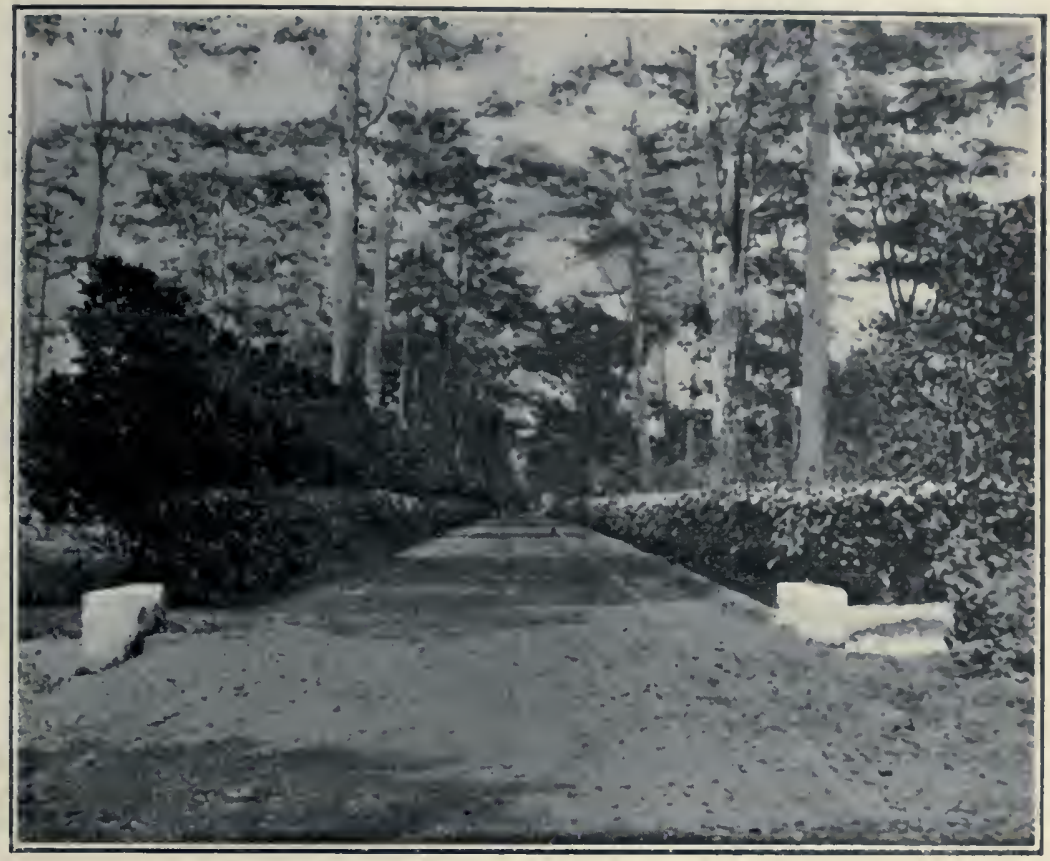

FIG. 1. -THE MAIN DRIVEWAY IN THE EXPERIMENT GARDEN.

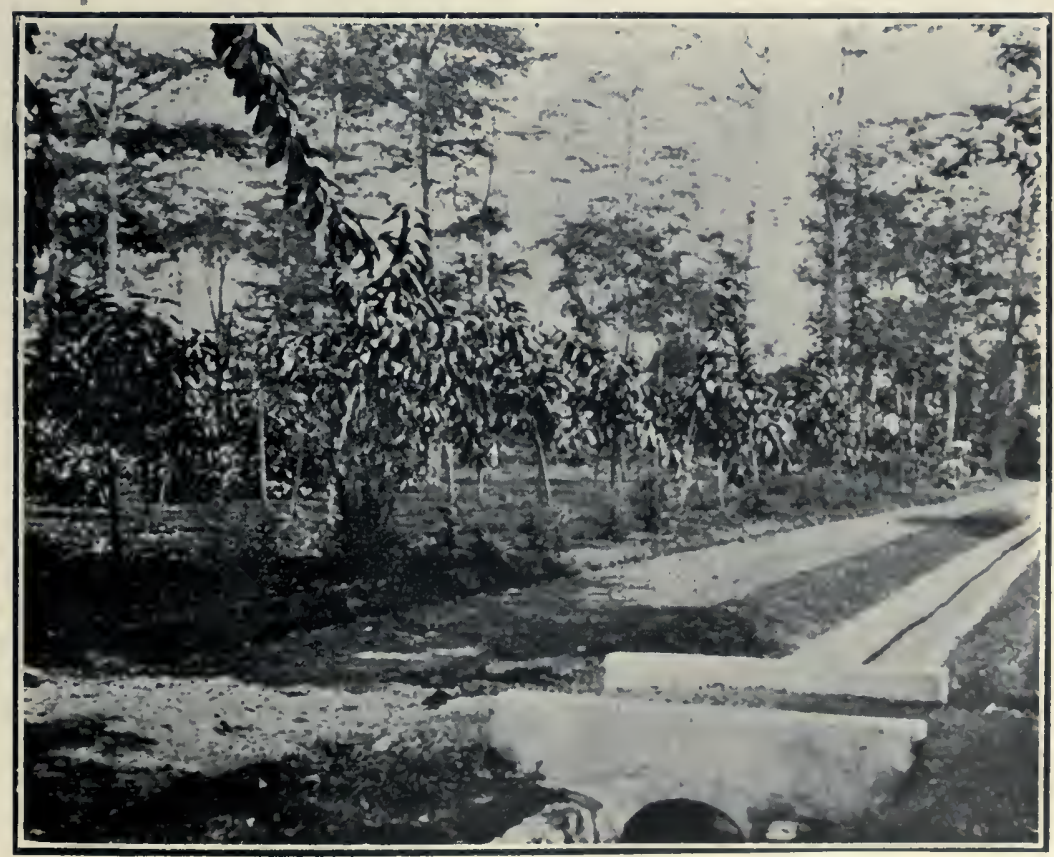

FIG. 2.-YOUNG PLANTS OF FICUS ELASTICA IN THE EXPERIMENT GARDEN. 
PLATE $X$.

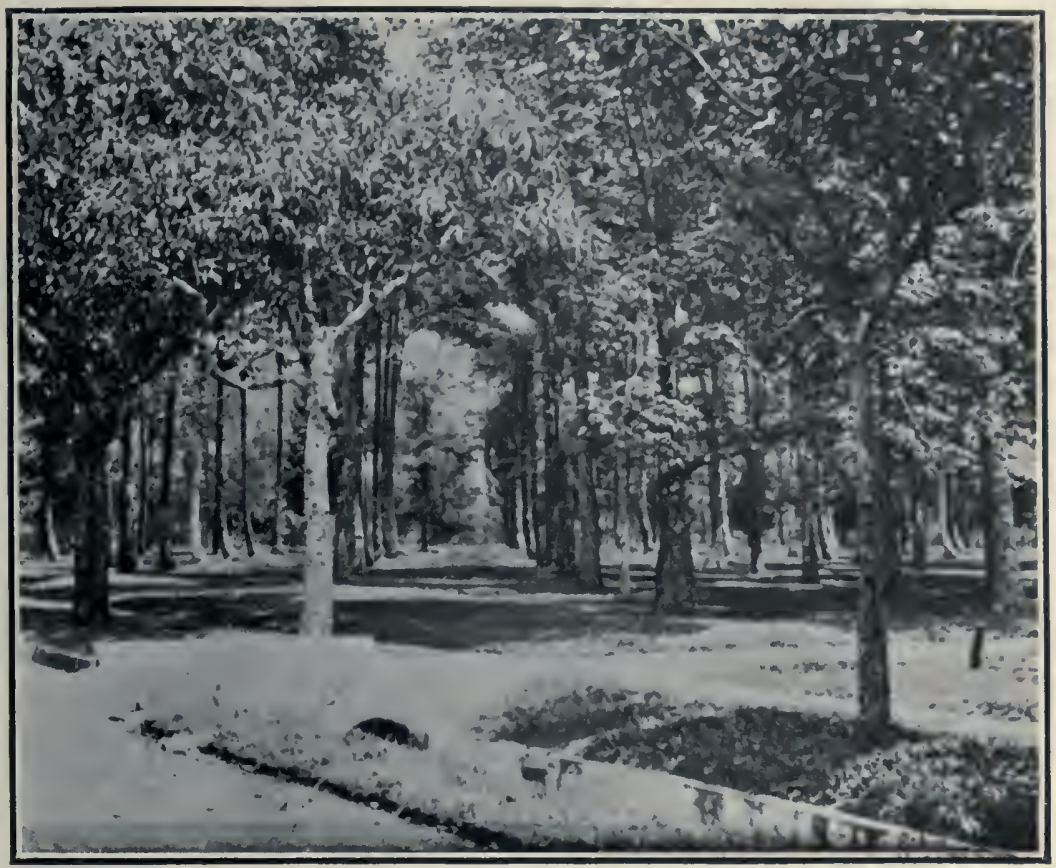

FIG. 1.-GUTTA-PERCHA TREES IN THE EXPERIMENT GARDEN.

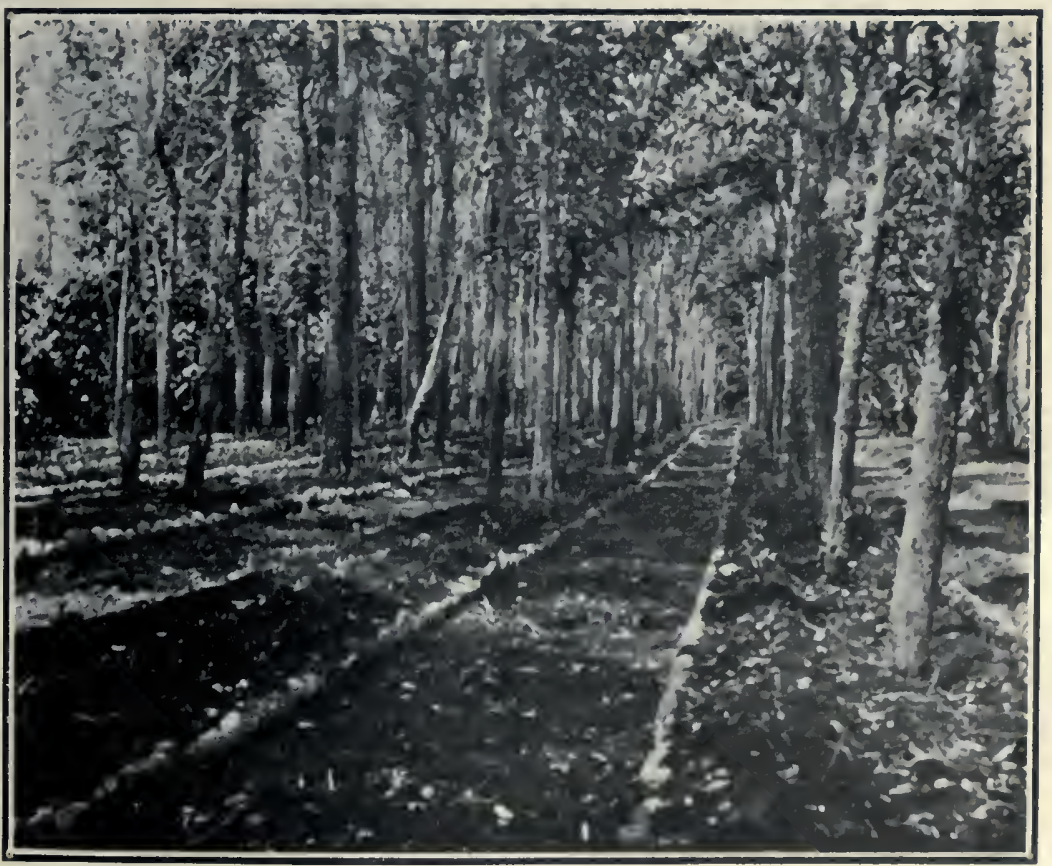

FIG. 2.-SHOWING THICKNESS OF PLANTING IN THE EXPERIMENT GARDEN. 



\section{N DEX.}

A.

Acalypha
Actephila
Adina
Aegiceras
Agraia
Ailanthus
Albizzia
Alchornea
Aleurites
Alstonia
Amoora
Anacardiacere...
Anacardium
Anaxagorea
Anona
Anonacere
Antidesma
A pocynacex
Araliacee
Ardisia
Arthrophyllum
Artocarpus
Asclepiadacere
Astronia
Averrhoa
Avicennia

13.

Baccaurea

Barringtonia

Bignoniacere

Bixa

Bixacer

Bombacese.

Borraginacere

Bridelia

Brucea

Bruguiera

Buchanania

Burseraceæ

$$
6425-6
$$

c.

\begin{tabular}{|c|c|c|}
\hline & & \\
\hline Page. & & Page. \\
\hline 32 & Caesalpinia - & 24 \\
\hline 29 & Callicarpa & 51 \\
\hline 53 & Calophyllum ...... & 39 \\
\hline 45 & Cananga & 20 \\
\hline 24 & Canarium ......... & 27 \\
\hline 28 & Casearia _...... & 41 \\
\hline 27 & Cassia ........ & 24 \\
\hline 23 & Ceiba ......- & 37 \\
\hline 31 & Celastracese_._- & 34 \\
\hline 32 & Celtis & 16 \\
\hline 48 & Cerbera & 49 \\
\hline 28 & Ceriops............... & 43 \\
\hline 33 & Chisocheton & 28 \\
\hline 33 & Cinnamounum ... & 21 \\
\hline 20 & Citrus ........... & 27 \\
\hline 20 & Claoxylon & 31 \\
\hline 19 & Clausena & 26 \\
\hline 30 & Cleidion & 31 \\
\hline 48 & Clerodendron & 52 \\
\hline 45 & Codizeum & 32 \\
\hline 46 & Colona _........... & 36 \\
\hline 45 & Combretaceæ ... & 44 \\
\hline 17 & Commersonia & 37 \\
\hline 49 & Compositre & 54 \\
\hline 45 & Coniferea & 15 \\
\hline 25 & Counaracere ..... & 22 \\
\hline 52 & Connarus _. & 22 \\
\hline & Convolvulacere & 50 \\
\hline 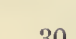 & Cordia & 50 \\
\hline 30 & Couthovia & 48 \\
\hline 42 & Croton & 30 \\
\hline 52 & 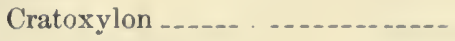 & 39 \\
\hline $\begin{array}{l}40 \\
40\end{array}$ & Cyclostemon $\ldots \ldots \ldots$ & 30 \\
\hline 37 & D. & \\
\hline 50 & Dalbergia_........ & 25 \\
\hline 30 & Datisacere _....... & 41 \\
\hline 27 & Decaspernum & 43 \\
\hline 43 & Dehaasia ......... & 21 \\
\hline 33 & Desmodiun _- & 25 \\
\hline 27 & Dillenia _._. & 39 \\
\hline
\end{tabular}


Page.

Dilleniaces:

Diospyros _..._.

Diplediscus

Jipterocarpacese ..............

Dipterocarpus

1)olichandrone

Donax

Duabanga

l)ysoxylum

\section{E.}

Ebenace:

Ehretia

Elacocarpacese

Elaevearpus

Ellatostachys

Erioglossum

Erythroxylacea -............

Erythroxylon

Euphorbia.

Eupborbiacese

Evodia

Fxcoecaria

\section{F.}

Fagacete

Fagraca

Ficus

Flacourtia

Flacourtiace:

G.

Garcinia

Gardenia

Cielonium

Cieunsia

(ileditschia

Giricidia_.

Glorhidion

Gilycosmis

(imelina

(inetacea

Gnetuu

Gonoraryum . ... -. -

Gioodeniacese -...

(iossypium - .................

Givettarcla

Guttefereat

Givinacrunthera

Gymnosporia . ..................

Gynotroches.

(iyrinopsis

47
II.

Page.

Harpullia

35

36

40

40

50

36

36

Heritiera _..._...

Heruandia _........._......... 21

Hernandiac'ex . _................. 21

Hibiscus ... 37

Homalanthus._._. 32

Homalium .... 41

Homonoia ........ 32

Hoya _....... 49

Hydnoearpus .

I.

I cacinacese _. .... . .

Illipe _............. 46

Indigofera ......... 24

I pomoea _... . . . . .

Ixora _....... 54

I.

Jambosal _. _. . - .

I..

Kibara _........... 21

Kleinhovia _............ 38

Koortersiodendron _...... 33

I.

Lagerstroemia ............ 42

Laurace:e _..._. . .

Lawsonia................ 42

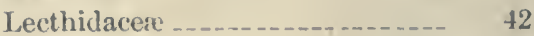

Leea _._. 36

Leguminusede _..._._. _........ 22

Lepidopetalum _.............. 35

lepiniopsis ................... 48

leucosyke................... 19

I.oganiacea _........... 48

I,ophopetalum _....... 34

Lumnitzera_..._ _ _......... 44

L Jumaia -......... 26

Lythraceie _....... t2

I.

Maba _.................. 47

Nacaranga _............. 31

Maesa _..._. 45

Malphigiacese _._._... 29

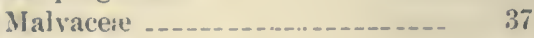

Mallotus _._._. 31

Mangiferal _....... 33

Marantacese _................. 16

Mayalea _............ 48

Melastomaceac _.......... 45

Meliacex -. 
Ielochia

Memecylon

Microglossa

Nicromelum

Minusops

Mitragne

Mitrephora

Monimiace:

Moracere

Morinda

Moringa

Moringacest

Muntingia

Mussaenda

Mlyristica

Myristicacere

Myrsinacese

Myrsine

Myrtace

N.

Nauclea

Nephelium

Nyctaginacere

o.

Octomeles

Olea

Oleaceæ

Otophora

Oxalidacen

P.

Palaquium

Purinarium

Parkia

Payena

Pemphis

Phaeanthus

Phaleria

Phoebe

Phyllanthus

Picrasma

Pinus

Pipturus

Pisonia

Pithecolobium

Pittosporacex

Pittosporum

Plectronia

Plumiera

Pongamia

Premna

Psidium

Psychotria
Page.

37 Pterocynbium

Page.

45

Pterospermum

Pygeum

Quercus

a.

Quisqualis

IR.

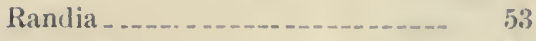

Rapanea -.._-_._. $46^{\circ}$

Rauwoltia ................. 49

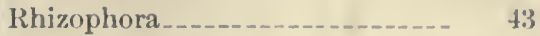

Rhizophoracere .............. 43

Rosacere_.............. 22

Rubiaceie _..._. _. 53

Rutacere_-.._-

Ryparosa _..._._.

Sandoricum _..__... 28

Sapindacete_........................ 34

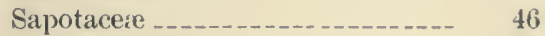

Sarcocephalus _................... 53

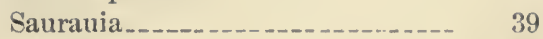

Sauropus _..................... 30

Scaevola_______._. 54

Scheftlera -........ 45

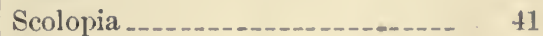

Semecarpus_._._. 33

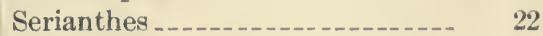

Shorea _._........ 40

Simarubacere - _..._._._._._._. 27

Sindora _._..................... 23

Sonneratia_.................... 42

Sonneratiaceæ ................ 42

Sophora _........................ 24

Staphyleacere _................... 34

Sterculia _.................. 38

Sterculiacere _.................... 37

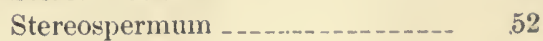

Streblus _._._._. 16

Strychnos _..._._. 48

Syzygium _._.

T.

Tabernaemontana_............... 49

Tamarindus _................... 24

Tarrietia _................ 38

Taxotrophis _..._._._._._._._. 16

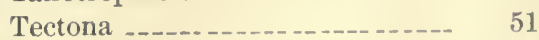

Terminalia .................... 44

Ternstrœmia _................ 39

Theacere _...

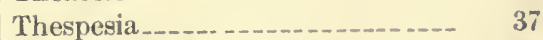

Thy melaeacer _............. 41 
Tiliacer

Page.

36

Timonius

Toona

Tournefortia

Trachelyspermum

Trema

Tricalysia

Triphasia

Tristillateia

Tristira

Turpinea

v.

Proge.

54 Terbenacee

50

Villebrunea

19

50 Vitacere -

$50 \mid$ Vitex _...

49 Vitis _.._

\begin{tabular}{l|l}
16 & Voacanga_._. \\
54 & 49
\end{tabular}

26. w.

29 Wallaceodendron _............ 23

35 Walsura

34 Wendlandia

U.

Wrightia

Ulinace:e

Unona

Urophyllum

$\mathbf{x}$

Urticacere

53 Xanthostemon

44

19 Xylocarpus

Uvaria

19 Xylopia

20

○ 


PLEASE DO NOT REMOVE

CARDS OR SLIPS FROM THIS POCKET

UNIVERSITY OF TORONTO LIBRARY

QK
367
M47
1903
C.1
BMED 
$=$ 